\title{
A Solar Sail Technology Application Mission for Analyzing the Earth's Geomagnetic Tail
}

by

\author{
David C. Prodger, B.Eng. \\ A thesis submitted to \\ the Faculty of Graduate Studies and Research \\ in partial fulfillment of \\ the requirements for the degree of \\ Master of Applied Science \\ Ottawa-Carleton Institute for \\ Mechanical and Aerospace Engineering \\ Department of \\ Mechanical and Aerospace Engineering \\ Carleton University \\ Ottawa, Ontario \\ June $19^{\text {th }}, 2002$
}

(C) Copyright

2002, David C. Prodger 
National Libran

of Canada

Acquisitions and

Bibliographic Services

395 Wellington Street

Otawa ON KIA ONA

Canada
Bibliothèque nationale

du Canada

Acquisitions et services bibliographiques

395, rue Wellington

Ottawa ON KIA ONA

Canada
Vour Flle Votre ratienance

Our Notre rêtrence
The author has granted a nonexclusive licence allowing the National Library of Canada to reproduce, loan, distribute or sell copies of this thesis in microform, paper or electronic formats.

The author retains ownership of the copyright in this thesis. Neither the thesis nor substantial extracts from it may be printed or otherwise reproduced without the author's permission.
L'auteur a accordé une licence non exclusive permettant à la Bibliothèque nationale du Canada de reproduire, prêter, distribuer ou vendre des copies de cette thèse sous la forme de microfiche/film, de reproduction sur papier ou sur format électronique.

L'auteur conserve la propriété du droit d'auteur qui protège cette thèse. $\mathrm{Ni}$ la thèse ni des extraits substantiels de celle-ci ne doivent être imprimés ou autrement reproduits sans son autorisation. 
The undersigned recommend to the Faculty of Graduate Studies and Research acceptance of the thesis

\section{A Solar Sail Technology Application Mission for Analyzing the Earth's Geomagnetic Tail submitted by \\ David C. Prodger, B.Eng.} in partial fulfillment of the requirements for the degree of Master of Applied Science

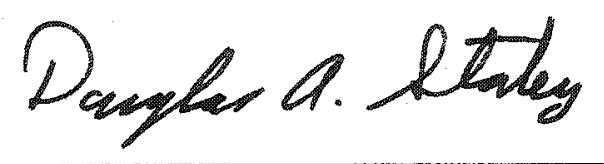

Thesis Supervisor

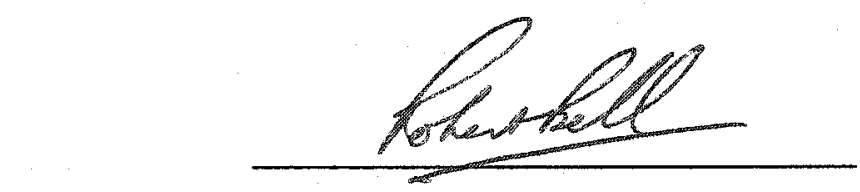

Chairman, Department of Mechanical and Aerospace Engineering

$$
\begin{aligned}
& \text { Carleton University } \\
& \text { June } 19^{\text {th }}, 2002
\end{aligned}
$$




\begin{abstract}
The concept of solar sailing had been the stuff of science fiction novels until the late 1970's when NASA considered using the technology in its Comet Halley rendezvous mission. Since then solar sailing has been gaining popularity as an alternative propulsion for rather unique mission proposals where the more conventional chemical propulsions are not viable. Investigations into the various aspects of the technology demonstrate that solar sailing could prove to be the answer for certain missions where chemical and even ion propulsion is limited.
\end{abstract}

The focus of this thesis is on the potential application of solar sailing as a means of propulsion and control for a satellite in a restricted three-body system. Given Canada's interest in the interaction of the solar wind with the Earth's magnetic field, a great deal could be learned by having a scientific payload "parked" permanently in the geomagnetic tail. This mission would only be possible if the spacecraft was able to generate thrust on a continuous basis which is considered one of the prime advantages of solar sailing. This thesis will cover the broad aspects of solar sail technology and then focus on solar sailing in a restricted three-body system. Finally, the spacecraft design will be covered at a conceptual level outlining the sail configuration and materials, the spacecraft mass budget, a potential launch vehicle and some navigation strategies once the spacecraft is in position. 


\section{Acknowledgements}

A research endeavour of this nature is not undertaken without the support of many individuals from academia as well as one's family. I would like to acknowledge the generous help and support of several people who assisted me in many ways so that I could complete my thesis.

My wife, Kimberly Beek, deserves my deepest gratitude for helping me through the thesis writing process as my mentor and my guide. She was my biggest supporter in helping to motivate me to put "pen to paper". She has always put everything else aside to edit the pages of this document or to just listen to my ideas. Thank-you.

I would also like to thank Professor Doug Staley, my thesis advisor, for helping me to develop this thesis and refine the scope so that I would not get too carried away with the details. As well I would like to thank Dr. Kieran Carroll who inspired me with his enthusiasm on the subject of solar sailing and provided me with the initial batch of articles to get me started. His valuable expertise and insight into the subject was much appreciated.

I would also like to acknowledge the support of the Canadian Forces and, more specifically, 410 Squadron, in allowing me the opportunity to take the education leave needed so that I could concentrate and complete this thesis.

Finally, thanks go to my parents who raised me to believe that I could accomplish anything with enough focus and effort. 


\section{Table of Contents}

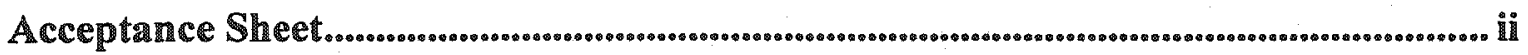

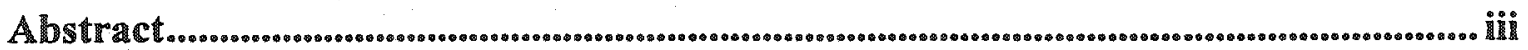

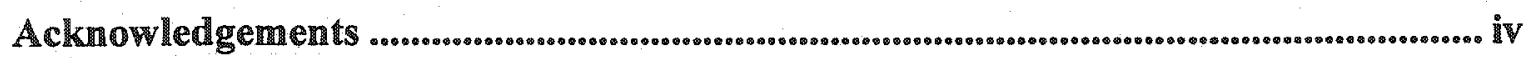

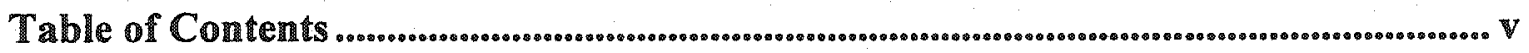

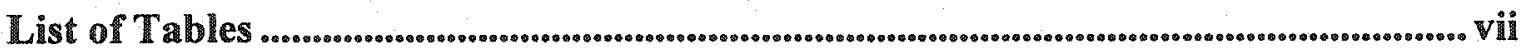

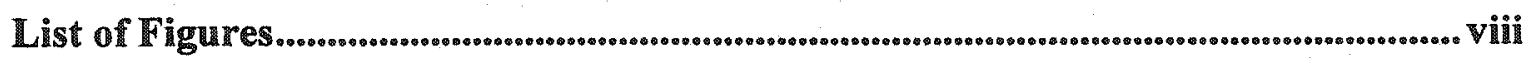

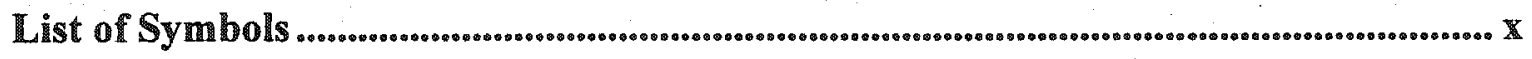

Introduction

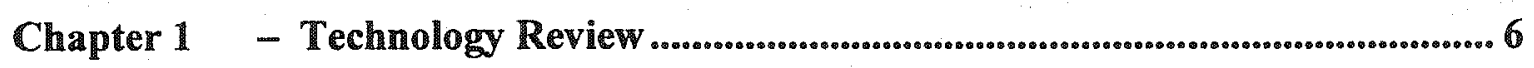

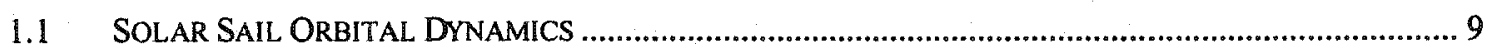

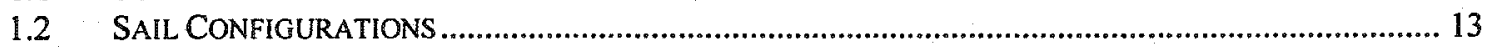

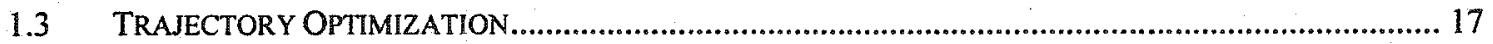

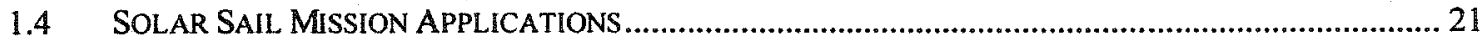

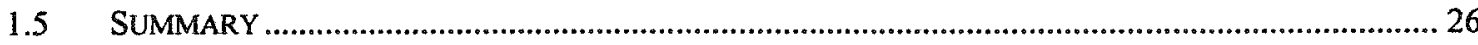

Chapter 2 - The Earth's Geomagnetic Environment............................................ 28

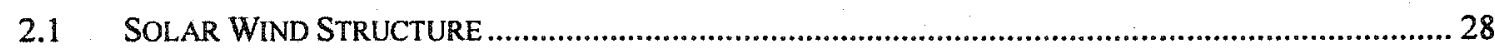

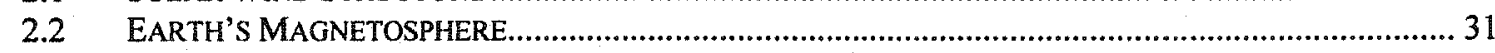

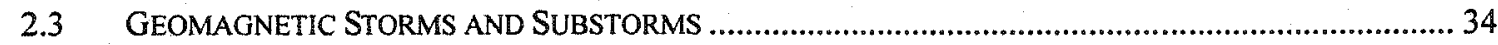

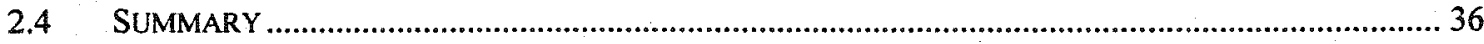

Chapter 3 - Solar Sail Propulsion................................................................................ 38

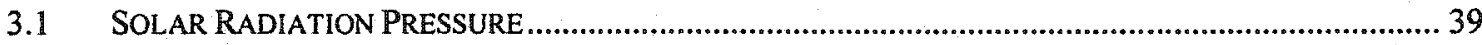

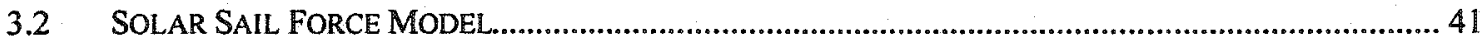

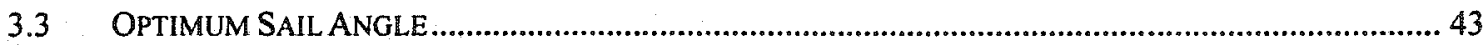

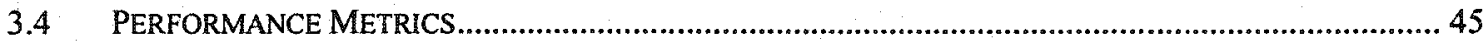

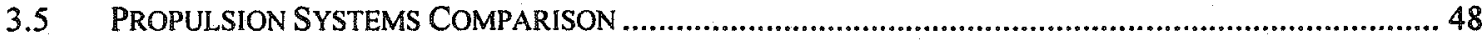

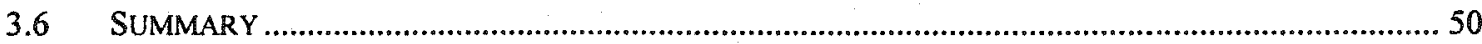

Chapter 4 - Solar Sail Dynamics in a Restricted Three-Body System ................ 52

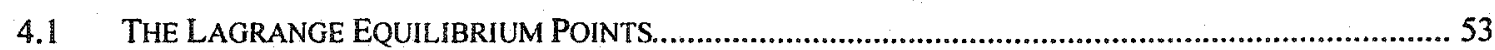

4.2 SOLAR SAILS IN RESTRICTED THREE-BODY SYSTEMS ............................................................59

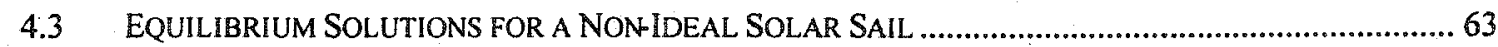

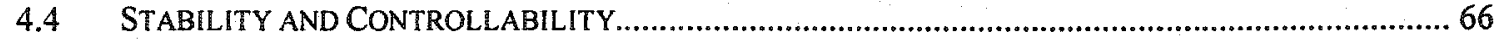

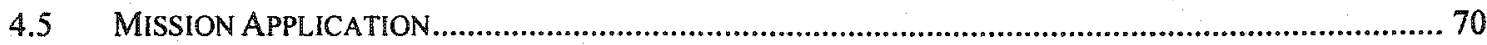

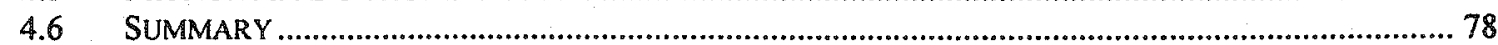




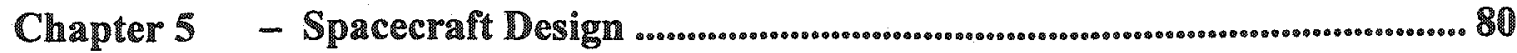

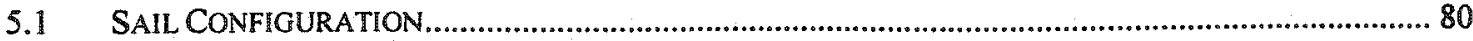

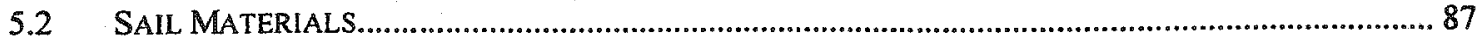

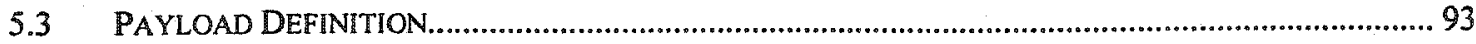

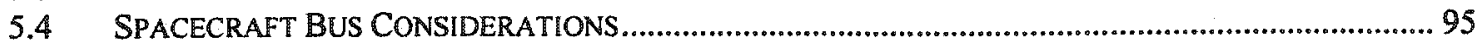

5.5 THE MAGELLANAEROSPACE CORPORATION SCISAT-1 PLATFORM ........................................98

5.6 FROM LAUNCH TO THE EQULLIBRIUM POSITION................................................................ 105

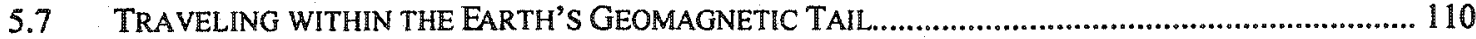

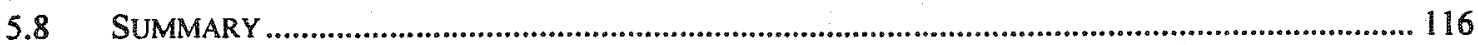

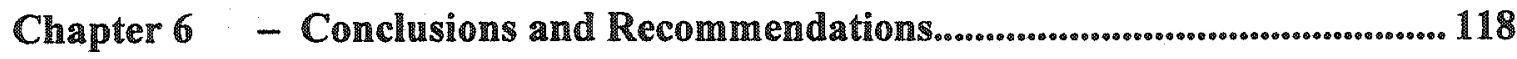

Annex 1 - Schematic of Scisat-1 Spacecraft ...................................................................... 122

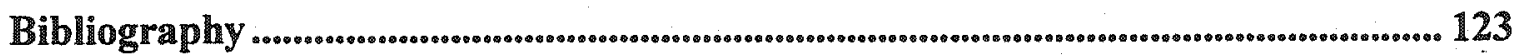




\section{List of Tables}

2.1 Summary of Geomagnetic Tail Dimensions ............... 36

4.1 Performance Metrics for Equilibrium Solutions in the Vicinity of Earth .... 62

4.2 Solar Sail Performance Metrics for an Ideal and Real Sail ... . . . . . . . . 79

5.1 Properties of Substrate Materials . . . . . . . . . . . . . . . . 88

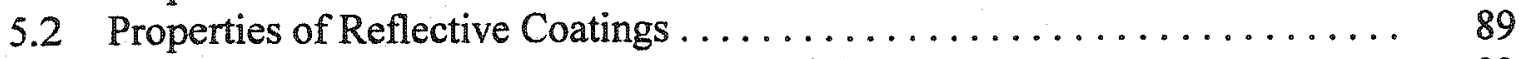

5.3 Properties of Rigidizable Laminate Material . . . . . . . . . . . . . . . . 92

5.4 Summary of Properties for Solar Sail Material Design . . . . . . . . . . . . 93

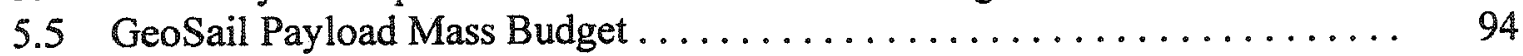

5.6 Mass and Power Budgets for Scisat-1 Spacecraft . . . . . . . . . . . . . . . . 99

5.7 Solar Sail Performance Metrics ........................ 102

5.8 Mass and Power Budgets for Solar Sail Propelled Scisat-1 Platform ....... 105 


\section{List of Figures}

2.1 Spiral Structure of the Interplanetary Magnetic Field . . . . . . . . . . . . . 29

2.2 Solar Magnetic Sector Structure Showing Magnetic Field Direction . . . . . . 29

2.3 Wavy Structure of Heliomagnetic Current Sheet................ 30

2.4 Cross Section of Earth's Magnetosphere ................... 31

2.5 Cross Section of the Magnetosphere Showing Various Current Systems .... . 32

2.6 Noon-midnight Cross Section of Magnetosphere and Geomagnetic Tail .... 33

2.7 Magnetic Field Topology with Neutral Line . . . . . . . . . . . . . . . . 34

2.8 Schematic of Plasma Flow within the Magnetosphere ........... 35

3.1 Light Forces on a Reflecting Sail . . . . . . . . . . . . . . . 42

3.2 Radial and Transverse Components of Solar Sail Force Vector . . . . . . . . . 44

3.3 Solar Sail Force Components . . . . . . . . . . . . . . . . . . . . . . 45

3.4 Comparison of Propulsion Systems with Solar Sail Payload Mass Fraction . $\quad 50$

4.1 Location of the Five Lagrange Equilibrium Points ............. 52

4.2 Restricted Three-body Problem Set Up ..................... 54

4.3 The Location of the Lagrange Equilibrium Points for a Mass Ratio of 0.01 . $\quad 58$

4.4 Vector Representation of a Solar Sail in a Restricted Three-body Problem . 59

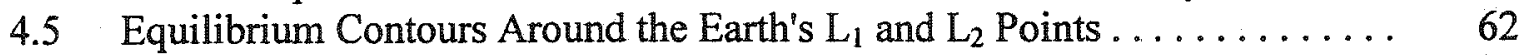

4.6 Sun-Earth Restricted Three-body Problem . . . . . . . . . . . . . . . 64

4.7 Equilibrium Contours of Sail Lightness Numbers ................ 65

4.8 Restricted Three-body Setup for a Solar Sail in the Sun-Earth System . . . . . 70

4.9 Characteristics of Acceleration Along the $x$ Axis . . . . . . . . . . . 73

4.10 Solar Sail Acceleration Characteristics at $100 \mathrm{R}_{\mathrm{E}} \ldots \ldots \ldots \ldots \ldots \ldots \ldots \ldots$

4.11 Solar Sail Accelerations Along the $y$ and $z$ Axes $\ldots \ldots \ldots \ldots \ldots \ldots$

5.1 Artist's Concept and Schematic of a Three-Axis Square Solar Sail Design. . 81

5.2 Deployment of a Three-Axis Stabilized Square Solar Sail . . . . . . . . . . . 81

5.3 Artist's Concept and Schematic of a Heliogyro. ............... 83

5.4 Cloverleaf Sail Geometry ........................... 85

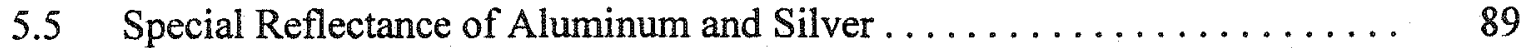

5.6 Plots of Equilibrium Temperature vs. Solar Distance . . . . . . . . . . . . . . 91

5.7 Cross Section of Sail Material for Geomagnetic Tail Mission . . . . . . . . . . 91

5.8 Cross Section of Bonded Solar Sail Film Panels. . . . . . . . . . . . . . . . . . 92

5.9 Artist's Rendering of the Scisat-1 Satellite .................. 98

5.10 Multi-view Schematic of Scisat-1 Spacecraft ................. 99

5.11 Diagram of Saillet Configuration ....................... 102

5.12 Rockot Launch Vehicle with Service Tower Schematic . . . . . . . . . . 106

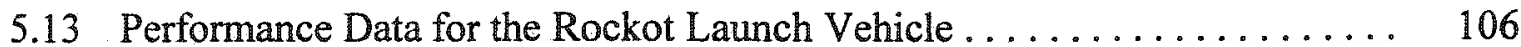

5.14 Solar Sail Trajectory Plot to Reach $L_{2}$ Point . . . . . . . . . . . . . . 108

5.15 Hypothetical Set Up of a Solar Sail Operating at $100 \mathrm{R}_{E} \ldots \ldots \ldots \ldots \ldots \ldots$

5.16 Steering Manoeuvre Used to Generate Transverse Force ........... 113

5.17 Depiction of Earth's Umbra . . . . . . . . . . . . . . . . . 113 
5.18 Depiction of Earth's Penumbra ..................... 114

5.19 Hypothetical Equilibrium Contours from Earth to $L_{2}$ Point ......... 115 


\section{List of Symbols}

\begin{tabular}{|c|c|}
\hline$E$ & Energy \\
\hline$h$ & Planck's constant \\
\hline$v$ & Energy frequency \\
\hline$m$ & Mass \\
\hline$m_{0}$ & Rest mass \\
\hline$c$ & Speed of light \\
\hline$p$ & Momentum \\
\hline$W$ & Energy flux \\
\hline$W_{E}$ & Energy flux at Earth's distance from the Sun \\
\hline$L_{s}$ & Solar luminosity \\
\hline$r$ & Distance from the Sun \\
\hline$r_{E}$ & Earth's distance from the Sun \\
\hline$A$ & Area \\
\hline$t$ & Time \\
\hline$F$ & Force \\
\hline$P$ & Pressure \\
\hline$A_{e f f}$ & Effective area \\
\hline$F_{i}$ & Incident force \\
\hline$F_{r}$ & Reflected force \\
\hline$\alpha$ & Sail tilt angle \\
\hline$F_{R}$ & Radial force \\
\hline$F_{T}$ & Transverse force \\
\hline$\sigma$ & Sail loading \\
\hline$a$ & Acceleration \\
\hline$a_{0}$ & Characteristic acceleration \\
\hline$\eta$ & Sail efficiency factor \\
\hline$\beta$ & Sail lightness number \\
\hline$a_{R}$ & Acceleration due to solar radiation pressure \\
\hline$a_{g}$ & Acceleration due to gravity \\
\hline$G$ & Gravitational constant of the Sun \\
\hline$M_{s}$ & Mass of the Sun \\
\hline$\Delta v$ & Change in speed \\
\hline$g$ & Gravitational acceleration \\
\hline$I_{s p}$ & Specific impulse \\
\hline$m_{0}$ & Mass of rocket with propellant \\
\hline$m_{p}$ & Mass of propellant \\
\hline$T$ & Mission duration \\
\hline & Chapter 4 \\
\hline$L_{1}, L_{2}, L_{3}, L_{4}, L_{5}$ & Lagrange equilibrium points \\
\hline$m_{1}, m_{2}$ & Masses of two bodies \\
\hline$\xi, \eta, \zeta$ & Coordinates for inertial frame \\
\hline$x, y, z$ & Coordinates for rotating frame \\
\hline
\end{tabular}

\section{Chapter 3}

Energy

Planck's constant

Energy frequency

Mass

Rest mass

Speed of light

Energy flux at Earth's distance from the Sun

Solar luminosity

Distance from the Sun

Earth's distance from the Sun

Area

Pressure

Effective area

Incident force

Reflected force

Sail tilt angle

ansverse force

Sail loading

Acceleration

Characteristic acceleration

Sail efficiency factor

to solar radiation pressure

Acceleration due to gravity

Gravitational constant of the Sun

Mass of the Sun

Change in speed

Gravitational acceleration

Specific impulse

Mass of rocket with propellant

Mass of propellant

Mission duration

Chapter 4

Lagrange equilibrium points

Coordinates for inertial frame

Coordinates for rotating frame 
$\vec{\Omega}$

$\vec{r}$

$\overrightarrow{r_{1}}$

$\vec{r}_{2}$

$\vec{a}_{s s}$

$G$

$x, y, z$

$\sigma_{r}$

$a_{s s,}$

$\beta_{r}$

$T$

$\tilde{r}$

$\tilde{\sigma}$

$\varepsilon_{f}$

Particle

Distance from $\mathrm{m}_{l}$ to $\mathrm{P}$

Distance from $m_{2}$ to $P$

Distance from center of mass to $P$

Mass of a body multiplied the gravitational constant Angular velocity

Time

Scalar function

Sail lightness number

Angular velocity

Solar radiation pressure acceleration vector

Gravitational potential

Sail normal unit vector

Vector from center of mass of two body system

Offset vector

Sail cone angle

Offset angle

Sail efficiency coefficient

Ideal sail lightness number

Coordinates of equilibrium positions

Small displacements

$n \times n$ matrix of constants

Displacement vector

Eigenvector

Eigenvalue

$n \times n$ unit matrix

Mass ratio of two body system

Mass of the Sun

Mass of the Earth

Mass of the spacecraft

Angular velocity vector

Vector from center of mass to spacecraft

Vector from Sun to spacecraft

Vector from Earth to spacecraft

Solar sail acceleration vector

Gravitational constant

Cartesian coordinates

Sail loading for a real sail

Characteristic acceleration for a real sail

Sail lightness number for a real sail

Chapter 5

Sail temperature

Reflectivity efficiency

Stefan-Boltzmann constant

Emissivity of front surface 


$\begin{array}{ll}\varepsilon_{b} & \text { Emissivity of back surface } \\ \sigma_{s p} & \text { Sail loading - spacecraft } \\ \sigma_{s s} & \text { Sail loading - solar sail } \\ m_{s p} & \text { Spacecraft mass } \\ m_{s s} & \text { Solar sail mass } \\ A_{s p} & \text { Spacecraft area } \\ A_{s s} & \text { Solar sail area } \\ p & \text { Gas pressure } \\ V & \text { Gas volume } \\ m & \text { Mass of the gas } \\ R & \text { Universal gas constant divided by molecular weight } \\ T & \text { Gas temperature }\end{array}$




\section{Introduction}

Canada has had a leading role in the investigation and research of the space environment since the launch of the Alouette and ISIS satellites in the 1960s. The efforts to date have been supported through funding by both government and industry so that a better understanding of "space weather" can help Canada and the international community safeguard its technological systems. The most visible example for the need to monitor and understand the solar wind and its interaction with the Earth's magnetic field occurred on the $13^{\text {th }}$ of March 1988 when the province of Quebec was "left without electrical power due to the temporary loss of $9450 \mathrm{MW}$ of capacity from the HydroQuebec power companyy grid. This massive power black-out was due to a geomagnetic storm inducing currents in power transmission lines which led to switchgear and transformer failures." Geomagnetic storms have an enormous effect not only on power grids but also on other ground systems, such as pipelines and undersea cables, satellites, radio communications systems, and navigations systems.

In a report submitted to the Canadian Space Agency on the $19^{\text {th }}$ of April, 1999 entitled Space Weather in Canada: A Plan of Action Under Long Term Space Plan III, the authors, William Liu, Robert Rankin and David Boteler, emphasized that scientific work is needed in three key areas to better understand the near-Earth space environment: 1) energy storage and release in the space environment; 2) magnetosphere-ionosphere coupling; and, 3) the electromagnetic response of the Earth. They stated that "In each

\footnotetext{
' Colin R. McInnes, Solar Sailing: Technology, Dynamics and Mission Applications, Praxis Publishing, Chichester, UK, 1999, p. 231.
} 
focal area is embedded some of the most intensely researched space science problems, such as the triggering mechanism for space weather storms, and the origin of auroral arcs and ionospheric disturbances."

Our solar system is infused with solar plasma which originates from the Sun and travels outward at the speed of light. This plasma or "solar wind" interacts with the planets and, more specifically, their magnetic fields. For the Earth, the interaction of the solar wind with its magnetic field creates an elongated, teardrop-shaped structure called the magnetosphere. The magnetosphere is populated by a wide energy range of charged particles which either increase or decrease in intensity depending upon the activities of the Sun. During periods of increased solar wind activities, "large currents flow in the magnetosphere, and large numbers of particles are injected into the ionosphere and at geostationary orbit. These conditions are the main cause of space weather effects on technological systems." Therefore, the study of the magnetosphere and its energy transportation processes would improve our understanding of "space weather" and how it contributes to the energy release phenomenon known as magnetic storms.

There are essentially two methods for gathering information on the space environment: 1) ground based and 2) space based. Canada has a very well established ground magnetic observatory system with CANOPUS and CANMOS, and plans on continuing to improve the system. While ground based measuring systems are very good at gathering information on magnetic field activity in the Earth's atmosphere, they cannot

\footnotetext{
${ }^{2}$ William Liu, Robert Rankin and David Boteler, Space Weather in Canada: A Plan of Action Under LTSP III, April 19, 1999, p. 3.

${ }^{3}$ Ibid. p. 6.
} 
measure activities "down stream" of the solar wind. For those measurements space based payloads must be used to analyze the structure of the magnetosphere and how it interacts with the solar wind. There have been several payloads launched into orbit focused on measuring the magnetic and plasma activities in the near-Earth magnetosphere but there have been very few launched to look at the distant tail section. As scientists discover more about the magnetosphere/ionosphere interaction, they find that greater understanding is required of the geomagnetic tail to get the entire picture of the magnetosphere structure. This is especially true with the formation of magnetic storms. Scientists are currently attempting to model the interaction of ions and magnetic field lines in the magnetosphere to better understand and hopefully predict the mechanisms involved in the development of magnetic storms.

While measurements continue to be taken for the near-Earth magnetosphere environment, there is a need for greater analysis of the geomagnetic tail. Canada is in an excellent position to sponsor a space based observation "platform", given its expertise in space plasma physics theory and in space environment modeling. The problem, however, lies in launching a payload into an orbit such that it remains in the geomagnetic tail. Traditional orbital mechanics dictate that any satellite launched into orbit would be in a continuous free fall about the Earth unless it possessed the ability to generate continuous thrust to counteract the gravitational effects of the Sun and Earth.

Another possibility exists, however, with the five Lagrange equilibrium points of a restricted three-body system. These points exist due to the balance of forces between. gravitational and centrifugal accelerations. For the Earth, the $\mathrm{L}_{2}$ equilibrium point exists 
at approximately 1.01 AU (Astronomical Unit) down stream of the solar wind and potentially within the geomagnetic tail. Unfortunately, the equilibrium point is unstable, meaning a spacecraft would need an active steering mechanism to maintain its position. Also, the spacecraft would be restricted to this position and unable to move without being attracted towards the Sun or Earth.

A propulsion technology that continues to gain greater attention in the space community is solar sailing. While Kepler postulated the existence of solar pressure in 1619, it was not until 1920 that Tsiolkovsky and Tsander conceived the concept of solar sailing. ${ }^{4}$ Solar sailing uses the momentum from the Sun's photons to produce a continuous thrust just as a sailing ship uses the wind to propel it through the water. Although relatively small, this propulsion can allow for some rather unconventional orbits and mission applications. If a solar sail spacecraft were to be positioned near the Earth's $\mathrm{L}_{2}$ equilibrium point, the continuous thrust produced by the sail would allow the spacecraft to move up the Earth-Sun line and further into the geomagnetic tail. Also, since the sail has the ability to change its orientation and therefore direct the thrust either in-plane or out-of-plane, it would be able to manoeuvre throughout a significant portion of the geomagnetic tail. In fact, because solar sailing does not expend any propellant, it has the potential to remain on-station for an indefinite period of time limited only by the component and sail material lifespan.

Over the years, interest has increased and a great deal has been written on solar sailing technology's various aspects and mission applications to the point where it is

\footnotetext{
${ }^{4}$ Colin R. McInnes, "Solar Sail Spacecraft: Historical Development and Mission Applications", Interdisciplinary Science Reviews, Vol. 20, No. 4, 1995, pp. 289-290.
} 
fairly mature as a concept. Proponents of the solar sailing technology do not suggest that it will replace traditional reaction mass-based missions but rather that it may be the answer for certain unique space mission applications. Placing a scientific payload in the Earth's geomagnetic tail for an extended period of time is an ideal application for solar sail technology.

The objective of this thesis is to articulate the basic design criteria required for a mission using a solar sail propelled spacecraft to fly to a parking orbit in the Earth's geomagnetic tail for the purpose of measuring ion interactions as they pertain to magnetic storm formation. The level of detail intended for this thesis is on the order of a Proof of Concept or a Preliminary Design Review. There is no intention to delve into detailed analysis of specific components of the spacecraft. In the following chapters various aspects of the mission application will be examined including: a technology review, the geomagnetic space environment, solar sail propulsion, solar sail dynamics in a restricted three-body system, and the spacecraft design. This thesis will prove that it is possible for a scientific payload to be "parked" in an equilibrium position within the Earth's geomagnetic tail using solar sail propulsion. 


\section{Chapter 1 - Technology Review}

The interest in solar sailing as a propulsion concept has captured the attention of the science community seemingly just in the past decade, however, work on the subject can be traced back to the Russian pioneers of astronautics. In his paper entitled "Solar Sail Spacecraft: Historical Development and Mission Applications," McInnes details the historical perspective of the concept and the key authors responsible for its development.

The theory of solar radiation pressure can be traced back to Johannes Kepler (1619) who proposed that a comet's tail was pushed outward by light from the Sun. However, this was a qualitative description and was not proven in an actual theory until James Maxwell. "Maxwell predicted the existence of radiation pressure in 1873 as a consequence of his theory of electromagnetic radiation." At the same time, Bartoli also proved solar radiation pressure existence through the second law of thermodynamics. The theory was proven experimentally by Lebedew in 1901 using a series of torsion balance experiments. During the early 1920s, two Russian scientists, Tsiolkovsky and Tsander both wrote of "using tremendous mirrors of very thin sheets" and "using the pressure of sunlight to attain cosmic velocities. ${ }^{.96}$ Tsiolkovsky is considered the Soviet father of astronautics and wrote primarily of propulsion using light, while Tsander wrote more specifically of practical solar sailing.

\footnotetext{
${ }^{5}$ Colin R. McInnes, "Solar Sail Spacecraft: Historical Development and Mission Applications", Interdisciplinary Science Reviews, Vol. 20, No. 4, 1995, p. 290.

${ }^{6}$ Colin R. Mclnnes, Solar Sailing: Technology, Dynamics and Mission Applications, Praxis Publishing, Chichester, UK, 1999, p. 2.
} 
Following the initial theoretical work by Tsiolkovsky and Tsander, there were no further developments until the late 1950s. In 1958, Richard Garwin examined solar sailing and demonstrated that it could be used for a round trip to Venus using commercially available materials. He was also the first to coin the phrase "solar sailing." Following this paper, Tsu (1959), and later London (1960), proved that solar sail trajectories were logarithmic spirals. Tsu developed the equations of motion for a solar sail that was only affected by the Sun's gravitational force (heliocentric). He then determined the logarithmic spiral angle and the optimum sail setting. Finally, he calculated the duration of solar sail trips to both Venus and Mars with the Earth as a starting point and compared these trip durations to those of chemical rockets. Tsu's conclusions state, "the solar sail seems to have important performance advantages over either chemical or ionic propulsion for trips to Mars or Venus." London developed more accurate solutions to T'su's equations and introduced the use of conic sections to better describe the orbit transfer time. However, he concluded by stating "neither the logarithmic spiral nor the conic section solution is optimum; other types of trajectories are undoubtedly necessary to utilize the sail most efficiently. ${ }^{9}$

During the 1970 s a solar sail design was being considered for a rendezvous mission with Halley's comet. While working at Battelle laboratories, Jerome Wright "discovered a trajectory which would allow a solar sail rendezvous with Comet Halley at its perihelion in the mid 1980s. The flight time of only 4 years would allow a late 1981

\footnotetext{
${ }^{7}$ T.C. Tsu, "Interplanetary Travel by Solar Sail", American Rocket Society Journal, Vol. 29, June 1959, p. 427.

${ }^{8}$ Howard S. London, "Some Exact Solutions of the Equations of Motion of a Solar Sail with Constant Sail Setting", American Rocket Society Journal, Vol. 30, February 1960, p. 200.
} 
or early 1982 launch." A formal proposal was put to NASA in September 1976 and by November the design project was funded. For the first time solar sailing was being considered as a viable propulsion technology for an actual mission. Over the next ten months the design team studied every facet of solar sailing. Initially the sail configuration was to be square $(800 \mathrm{~m} \times 800 \mathrm{~m})$ but this was discarded due to the expected high risk during sail deployment. Instead a spin-stabilized heliogyro designed by Richard MacNeal was adopted. Earlier, NASA had contracted MacNeal to publish a report entitled "Structural Dynamics of the Heliogyro" which he submitted in May of 1971. The heliogyro design proved to be much less risky during deployment because the "blades" were simply unrolled from rollers. MacNeal's report is also very thorough and covers the heliogyro design in detail including: the equations of motion, uncoupled vibration modes, damping devices, blade retention system, forces due to solar illumination, mechaniçal coupling due to blade deflections, chordwise curl and dynamic response during manoeuvres. ${ }^{10}$ Unfortunately, due to internal politics, NASA chose to pursue a rendezvous mission using solar electric propulsion instead of a solar sail. This occurred in September 1977. "Although discarded by NASA for the near term mission applications, the design studies of the mid 1970s did stimulate worldwide interest in solar sail technology."

The public interest and media exposure generated by a Comet Halley rendezvous mission did a great deal to generate interest for solar sail technology in the scientific

\footnotetext{
${ }^{9}$ Colin R. Mclnnes, "Solar Sail Spacecraft: Historical Development and Mission Applications", Interdisciplinary Science Reviews, Vol. 20, No. 4, 1995, p. 290.

${ }^{10}$ Richard H. MacNeal, Structural Dynamics of the Heliogyro, NASA Contractor Report CR-1745, May 1971, p. iii.

"Mclnnes, p. 291.
} 
community as well. During the 1980 s several groups formed dedicated to the promotion of solar sail technology. The World Space Foundation, based in California and made up of Jet Propulsion Laboratory engineers, attempted to raise private funds to launch a demonstration solar sail flight. The Union pour la promotion de Propulsion Photonique in Toulouse was formed in 1981 and proposed a moon race to promote solar sailing. "Both of these groups were joined by the Solar Sail Union of Japan in 1985 and proposed that the moon race be adopted as a project for the International Space Year in 1992."12

The interest generated by the Comet Halley rendezvous and the moon race proposals has resulted in a great deal of research and development in all aspects of solar sailing technology over the past two decades. Articles and books have been written detailing research in the following categories: solar sail orbital dynamics, sail configurations, trajectory optimization, mission applications and solar sail materials.

\subsection{Solar Sail Orbital Dynamics}

Solar sail orbital dynamics are quite different than the traditional Keplerian dynamics due to the continuous thrust imposed by the Sun's photons. Tsu and others developed equations of motion showing that a solar sail would follow a logarithmic spiral if in a heliocentric orbit. In fact a solar sail can either spiral outwards or inwards depending upon the orientation of the sail. This would make it possible to travel to Mars, as an example, either to fly-by or orbit the planet, and then return.

Forward proposed a very different kind of orbit in his paper entitled "Lightlevitated Geostationary Cylindrical Orbits." He demonstrated that a solar sail could be

\footnotetext{
${ }^{12}$ Mclinnes, p. 291.
} 
"used to levitate a communication satellite in a cylindrical orbit above or below the nominal equatorial earth-synchronous orbit."13 By "levitating" spacecraft above the equatorial plane, it would be possible to stack several of them at the same longitude and, therefore, increase the number of geostationary slots available for communications satellites. Forward went on to expand his concept by suggesting that perforated light sails would decrease the mass to area ratio of the solar sail and, therefore, increase the distance that the spacecraft can be "stacked" poleward. This may also allow geostationary orbiting spacecraft to communicate with Earth's polar regions. ${ }^{14}$ The concept of "levitating" a spacecraft using a solar sail led Forward to his most important work entitled "Statite: A Spacecraft That Does Not Orbit." Using a force balance analysis technique, he derived the equations of motion for a satellite that is located near the Lagrange equilibrium points. Since the equilibrium points are the areas which perfectly balance the Sun's gravitational force, the Earth's gravitational force, and the centrifugal force of the system, a solar sail would add an extra force component, allowing "equilibrium contours" depending upon the sail's mass to area ratio. This unique type of orbit would also allow the spacecraft to essentially fly in formation with the Earth throughout the year. ${ }^{15}$

Perhaps the author who has been most prolific concerning the dynamics of solar sailing is Colin R. McInnes. Beginning in the late 1980s until the present, McInnes has written many articles and most recently a book on the subject of solar sailing and its

\footnotetext{
${ }^{13}$ Robert L. Forward, "Light-levitated Geostationary Cylindrical Orbits", Journal of Astronautical Sciences, Vol. 24, No. 1, January-March 1981, p. 79.

${ }^{14}$ Forward, "Light-levitated Geostationary Cylindrical Orbits Using Perforated Light Sails", Journal of Astronautical Sciences, Vol. 32, No. 2, April-June 1984, p. 221.

${ }^{15}$ Forward, "Statite: A Spacecraft That Does Not Orbit", Journal of Spacecraft and Rockets, Vol. 28, No. 5, September-October, 1991 , p. 609.
} 
potential mission applications. From a primarily vector analysis perspective, he has developed the equations of motion for solar sails in a number of different orbits. Based on the paper by Van der Ha and Modi entitled "Long-term Evaluation of ThreeDimensional Heliocentric Solar Sail Trajectories with Arbitrary Fixed Sail Setting," McInnes has provided detailed analysis for Sun-centered orbits including: circular orbits, elliptical orbits, rectilinear orbits, escape orbits and logarithmic spiral trajectories. He has also addressed the issue of orbit inclination by using a "cranking" orbit to manoeuvre the spacecraft into a polar orbit about the Sun. ${ }^{16}$ McInnes has also analyzed planetcentered orbits and "in particular Earth escape trajectories [were] investigated using a variety of simple sail-steering laws." ${ }^{, 17}$ He also related the steering laws to minimum time escape trajectories from a planet. Finally, he provided a planet-centered orbit solution using the Hamilton-Jacobi method. ${ }^{18}$

McInnes also expanded upon Forward's work in the field of Non-Keplerian orbits. While Forward focused on a solar sail spacecraft balanced within the Earth-Sun system, McInnes developed the equations for a purely Sun-centered "Halo" orbit. He also demonstrated that there was a family of orbits termed Types I, II or III and he discussed the stability for each orbit type showing that there are regions of instability. To address the issue of orbit controllability, McInnes showed that the "system is indeed controllable using state feedback to the sail pitch attitude alone." 19 Finally, he discussed the issue of "patched orbits" and how it is possible for a spacecraft to move from one

\footnotetext{
${ }^{16}$ Colin R. McInnes, Solar Sailing: Technology, Dynamics and Mission Applications, Praxis Publishing, Chichester, UK, 1999, pp.112-150.

${ }_{18}^{17}$ Ibid, p. 151.

${ }^{18}$ Ibid, pp. 150-168.

${ }^{19}$ Ibid, p. 188.
} 
halo orbit to another, and even to a traditional Keplerian orbit if desired. ${ }^{20}$

For planet-centered non-Keplerian orbits, McInnes followed the same logical development as he did for the Sun-centered section. He demonstrated that a family of orbit types also existed for a planet-centered orbit and that there are regions of instability associated with each orbit type. He also discussed the controllability of the spacecraft in the regions of instability and proved that it is indeed controllable for all orbit types. Finally, McInnes discussed "patched orbits" and demonstrated that it is possible to patch to both a Keplerian orbit and a non-Keplerian orbit. ${ }^{21}$

For solar sails in restricted three-body systems, McInnes employed vector analysis to develop the equilibrium solutions first demonstrated by Forward. McInnes has provided the solutions for the Earth-Sun system and demonstrated that the equilibrium solutions are, in general, unstable. He also mentioned that, while the solutions are unstable, they are completely controllable using feedback to the sail attitude. McInnes then demonstrated the effect of a "real" sail model on the equilibrium contours showing that a non-ideal sail would have reduced performance due to the absorption of the sail material. ${ }^{22}$

While McInnes worked predominantly with an ideal solar sail model, Shvartsburg et al. expanded upon the halo orbit concept by introducing a sail with absorption. In a series of papers, Shvartsburg looked at both the heliocentric and geocentric halo orbits and compared an ideal sail with a less efficient one. For the heliocentric case, Molostov

\footnotetext{
${ }^{20}$ Ibid, pp. 173-196.

${ }^{21}$ Ibid, pp. 196-213.

22 Ibid, pp. 214-227.
} 
and Shvartsburg concluded, "absorption causes no qualitative changes in these halos.

The effect of the absorption reduces to a distortion of the ideal case."23 Due consideration must be given to the reflective and absorption qualities of the sail material when considering the mission application. The same is true for the geocentric case. Shvartsburg concluded: "for polar-synchronous halos, especially those with considerable elevations,...it is appropriate to provide the sail with the best reflective properties. All the same, many orbits cannot be obtained in principle or in practice (because of the extremely high sail efficiency required). ${ }^{, 24}$ Therefore, sail absorption may play a role in limiting the possible halo orbits that may be achieved, depending on the required sail efficiency. In a more recent paper entitled "Three-Dimensional Solar Sails," Shvartsburg explored the stability of a compound solar sail, one that incorporates both plane and cylindrical sections. He concluded that "the proposed compound sail is characterized by the passive stability of the levitation regime in any heliocentric orbit. ... The plane section determines the thrust and the cylindrical section provides the stability. ${ }^{, 25}$ He goes on to state that "A three-dimensional sail stabilized at the Lagrange point $L_{1}$ or other equilibrium points located along the Sun-Earth direction could have numerous applications." 26

\subsection{Sail Configurations}

Solar sail configurations are another area of research that has captured the interest

\footnotetext{
${ }^{23}$ A.A. Molostov and A.A. Shvartsburg, "Heliocentric Halos for a Solar Sail with Absorption", Soviet Physics Doklady, Vol. 37, No. 3, March 1992, p. 152.

${ }^{24}$ A.A. Shvartsburg, "Geocentric Halos for a Solar Sail with Absorption", Soviet Physics Doklady, Vol. 38 , No. 2, February 1993, p. 88.

${ }^{25}$ Shvartsburg, "Three-Dimensional Solar Sails: Passive Stability of Levitation and Slow Orbital Motion", Cosmic Research, Vol. 35, No. 2, 1997, p. 187.

${ }^{26}$ Ibid, p. 187.
} 
of the scientific community. Sail configurations come in a wide variety of styles depending upon the mission application. In his book entitled Space Sailing, Jerome Wright describes a variety of possible sail configurations from a square-rigged style to a heliogyro design. Although most designs are described from a qualitative viewpoint, he does mention that "only the square-rigged ship and the heliogyro have undergone substantial design evaluations," 27 referring to the Jet Propulsion Lab's efforts for the Comet Halley rendezvous.

As mentioned previously, MacNeal was the inventor of the heliogyro design and provided a great deal of information on its characteristics and control. In the conclusion to his report, MacNeal states that there are "no structural dynamic reasons to doubt the feasibility of the Heliogyro in sizes up to $10,000 \mathrm{ft}$ radius. ${ }^{928}$ Another very detailed heliogyro design was conducted by Blomquist as part of MIT's submission to the Christopher Columbus Quincentenary Race to Mars. This design incorporated a unique method of controlling the blade pitch using piezo film. The report considered many different parameters for the heliogyro and concluded with two suggested spacecraft design choices: one for the Pegasus launch vehicle and one for the Ariane launch vehicle. ${ }^{29}$ The amount of research into this configuration is very extensive which suggests that the heliogyro may be the most mature solar sail design and will need to be given due consideration for the geomagnetic tail mission application.

\footnotetext{
${ }^{27}$ Jerome L. Wright, Space Sailing, Gordon and Breach Science Publishers, The Netherlands, 1992, p.58.

${ }^{28}$ Richard H. MacNeal, "Structural Dynamics of the Heliogyro", NASA Contractor Report 1745, MacNealSchwendler Corporation, May 1971, p. 60.

${ }^{29}$ Richard S. Blomquist, "Design Study of a Solid-State Heliogyro Solar Sail", Master of Science Thesis, Massachusetts Institute of Technology, August 1990, pp. 151-152.
} 
P.D. Fieseler published a paper outlining a sail configuration that allows solar sailing in low earth orbit. Due to the fact that a solar sail must be oriented towards the Sun, it is usually affected a great deal by atmospheric drag. In fact, a minimum altitude for solar sail operation in the Earth's outer atmosphere is 1000 kilometers. ${ }^{30}$ Fieseler's design incorporates small ramps or slats in the sail material so that the sail may be placed in a low drag configuration and still generate some "along-track" thrust to enable a slow acceleration to Earth escape velocity. His analysis has demonstrated that it may be possible to deploy a solar sail as low as 350 kilometers and still achieve Earth escape. ${ }^{31}$

Another solar sail design was submitted for the Christopher Columbus 500 Space Sail Cup by the Canadian Space Society. This unique configuration looks like a large square or hexagon with centralized payload and four or six sail sections. The sail sections are made up of strips of material, called vanes, joined together to work like large Venetian blinds. To steer the sail, one or more of the sail section vanes are turned parallel to the Sun's incident radiation thereby creating a torque from the remaining sections. Although at a very preliminary stage of development, the design allows the spacecraft to be turned very quickly due to the high attitude authority from the individual section control. ${ }^{32}$

One technology that may become very beneficial for solar sailing is the concept of chemically rigidized expandables. In their paper entitled "Lobed Solar Sails for a

\footnotetext{
${ }^{30}$ P.D. Fieseler, "A. Method for Solar Sailing in a Low Earth Orbit", Acta Astronautica, Vol. 43, No. 9-10, 1998, p. 532.

${ }^{31}$ Ibid, p. 541.

${ }^{32} \longrightarrow$ Supplementary Notes for AER407F Spacecraft Design Course adapted from the interim design notes of the Canadian Space Society Solar Sail Design Team, September 19, 1989, Rev. 1.02, p. 16.
} 
Small Mission to the Asteroids," Bernasconi and Zurbuchen proposed a new type of sail design. The design incorporates four lobes or saillets connected to a central control unit/payload where each lobe is held rigid by an expandable hoop. The spacecraft is essentially in the shape of a cloverleaf where each "leaf" is independently controlled, allowing for optimum manoeuvrability. ${ }^{33}$ Given the recent successful launch of the Inflatable Antenna Experiment (IAE) on space shuttle Mission 77, this rigidized expandables technology may prove to be quite advantageous for solar sailing if it helps to minimize the sail deployment risks. ${ }^{34}$

Genta and Brusa proposed two other types of sail configurations which used a hydrostatic beam. Based on an article by Cassenti, Matloff and Strobl entitled "The Structural Response and Stability of Interstellar Solar Sails," Genta and Brusa suggested a modification to the "parachute" and "pillow" type of solar sail designs. For both configurations, a pressurized hydrostatic beam is used to keep the sail rigid and to relieve all compressive stresses, since excessive compression could lead to sail collapse. The authors were also concerned with designing the lightest sail structure possible. Using an inflatable hydrostatic beam helps to fulfill that design requirement. The advantages of the parachute sail are: simple structure, easy deployment, not affected by temperature variations, easily controlled by shifting its center of mass and fast roll velocities are possible. The only disadvantage is that the payload is exposed to the sunlight and,

\footnotetext{
${ }^{33}$ Marco C. Bernasconi \& Thomas Zurbuchen, "Lobed Solar Sails for a Small Mission to the Asteroids", Acta Astronautica, Vol. 35, Supplement, 1995, p. 648.

${ }^{34}$ Michael A. Dornheim, "Inflatable Structures Taking Flight", Aviation Week and Space Technology, Vol. 150 No. 4, 25 Jan 99, pp. 60-62.
} 
therefore, a close solar pass would not be possible." The "pillow" solar sail has the same advantages as the parachute design except that it helps to protect the payload so that a close solar pass is possible, but the control response is much slower. ${ }^{36}$

\subsection{Trajectory Optimization}

Although solar sailing produces a continuous thrust, there has been some concern that the amount of acceleration would be so small that mission times could become excessive. Therefore, several methods have been investigated to attempt to optimize the interplanetary trajectories and to demonstrate that a solar sail could be viable for planetary exploration missions.

In his paper entitled, "Optimum Solar-Sail Interplanetary Trajectories", Sauer built on the work of Zhukov and Lebedev ${ }^{37}$ by using the calculus of variations to optimize trajectories for solar sail missions to Venus, Mars, Mercury and an asteroid flyby. Sauer was the first researcher to optimize the trajectories in three-dimensions and "include inclined, elliptic orbits for the launch and target planets." ${ }^{38}$ In his conclusion, Sauer stated that "solar -sail spacecraft represents a viable option for the exploration of the solar system in the future." 39

Building on Sauer's work and a journal article entitled "Escape from Planetary

\footnotetext{
${ }^{35}$ G. Genta and E. Brusa, "The Parachute Sail with Hydrostatic Beam: A New Concept for Solar Sailing", Acta Astronautica, Vol. 44, Nos. 2-4, 1999, pp. 133-140.

${ }^{36}$ G. Genta and E. Brusa, "The Aurora Project: A New Sail Layout", Acta Astronautica, Vol. 44, Nos. 2-4, 1999, pp. 141-146.

${ }^{37}$ Zhukov and Lebedev, Variational Problem of Transfer between Heliocentric Orbits by Means of SolarSail", Cosmic Research, Vol. 2, No. 1, 1964, pp. 41-44.

${ }^{38}$ Carl G. Sauer Jr., "Optimum Solar-Sail Interplanetary Trajectories", AIAA/AAS Astrodynamics Conference, San Diego, CA, August 18-20, 1976, p. 1.

${ }^{39}$ Ibid. p. 8.
} 
Gravitational Fields by Use of Solar Sails" by Sands, Sackett and Edelbaum produced a computer program called SUNSPOT to determine optimal escape trajectories for solar sails. The computer program "can calculate solar sail planetocentric orbit transfers and trajectories to near escape condition.... Features include the ability to calculate trajectories about the four inner planets, and to include shadowing and Earth's oblateness. ${ }^{40}$ Sackett and Edelbaum also included some realistic considerations in their program such as an accurate solar sail model and a subroutine to ensure the spacecraft did not impact the planet to calculate the most optimized but "realistic" trajectory.

Since trajectory optimization may be solved by different techniques, Subba Rao and Ramanan have presented an alternative method in their paper entitled "Optimal Three-Dimensional Heliócentric Solar-Sail Rendezvous Transfer Trajectories”. Like Sauer, they follow the work of Zhukov and Lebedev by formulating the problem through a set of autonomous variables and then converting to a two-point boundary value problem utilizing Pontryagin's principle. However, the solution is "attempted through a version of the Controlled Random Search (CRS) optimization technique." ${ }^{41}$ While Subba Rao and Ramanan considered their technique to be an alternative to Sauer's, they also stated that it "offers a better understanding of the evolution of the transfer trajectory." ${ }^{A 2}$ Yet another method is offered by Simon and Zakharov in their paper entitled "Optimization of Interplanetary Trajectories with Solar Sail." The paper "describes the application of a modified indirect method to the solution of an optimal interplanetary solar-sail transfer.

\footnotetext{
${ }^{40}$ Lester L. Sackett and Theodore N. Edelbaum, "Optimal Solar Sail Spiral to Escape", AAS/AIAA Astrodynamics Conference, Grand Teton National Park, Wyoming, September 7-9, 1977, p. 60.

${ }^{41}$ P.V. Subba Rao and R.V. Ramanan, "Optimal Three-Dimensional Heliocentric Solar-Sail Transfer Trajectories", Acta Astronautica, Vol. 29, No. 5, 1993, p. 341.

${ }^{42} \mathrm{Ibid}$, p. 341.
} 
This method is related to the class of successive approximation methods and based on the continuation imbedding concept. ${ }^{943}$ This method attempts to solve the sensitive twopoint boundary value problem so that optimal control angles of the sail may be used to steer the spacecraft from Earth escape to an interplanetary trajectory and rendezvous effectively with the target planet. According to Simon and Zakharov, the method is "recognized as one of the most effective numerical techniques for the solution of numerically sensitive boundary-value problems of trajectory optimization and optimal control., ${ }^{, 44}$

Several authors have proposed optimized solutions for specific mission applications. Koblik et al. have proposed optimized trajectories into near-Sun orbits with a restriction on the sail temperature as a main consideration. Their work described several types of trajectories and the resulting duration in days required to obtain the desired orbit (e.g. circular, near-heliostationary etc.). ${ }^{45}$ Fekete et al. have presented a trajectory design for a solar sail launch from low-Earth orbit to an intercept location behind the moon. This paper was prepared as part of the MIT design team's efforts to produce a solar sail design for the Columbus 500 Space Sail Cup. The design included each stage of the transfer from a low Earth orbit to an intercept trajectory behind the moon. ${ }^{46}$

\footnotetext{
${ }^{43} \mathrm{~K}$. Simon and Y. Zakharov, "Optimization of Interplanetary Trajectories with Solar Sail",Space Technology, Vol. 16, No. 5/6, 1996, p. 382.

${ }^{44}$ Ibid, p. 382.

${ }^{45}$ V.V. Koblik, E.N. Polyakhova, L.L. Sokolov and A.S. Shmyrov, "Controlled Solar Sailing Transfer Flights into Near-Sun Orbits under Restrictions on Sail Temperature", Cosmic Research, Vol. 34, No. 6, 1996, pp. 572-578.

${ }^{46}$ Thomas A. Fekete, Lester L. Sackett and Andreas H. von Flotow, "Trajectory Design for Solar Sailing from Low-Earth Orbit to the Moon", AAS-92-184, AIAA Spaceflight Mechanics Meeting, February 1992, pp. 1083-1094.
} 
In an article entitled, "Near Minimum-Time Trajectories for Solar Sails," Otten and McInnes demonstrate that complex steering laws are not necessary for minimumtime trajectories. Using fixed attitude steering from an Earth to Mars trajectory means that very simple steering laws would be required to achieve the objective. In fact, Otten and McInnes concluded that "using four fixed-sail attitudes during the entire trajectory, the transfer time is increased by only 10 days. ${ }^{, 47}$ This means that solar sail steering does not have to be continuous changes in sail position to optimize the trajectory. A finite number of fixed sail positions can obtain nearly the same results with a minimal penalty.

One article that pertains directly to this thesis is entitled "Minimum Time Solar Sailing From Geosynchronous Orbit to the Sun-Earth $\mathrm{L}_{2}$ Point"' by Hur and Bryson. The authors break the trajectory problem down and "a combined method of local optimization and single shooting was used to solve the minimum-time transfer problem." ${ }^{\text {48 }}$ The local optimization portion of the trajectory involves a slow spiral away from Earth and an increase in the spacecraft's energy state. The single shooting method refers to the transfer from the last perigee to the $\mathrm{L}_{2}$ equilibrium point. Hur and Bryson found that with a given sail characteristic acceleration of $0.1 \mathrm{~mm} / \mathrm{s}^{2}$, the transfer time to the $\mathrm{L}_{2}$ point would be 2.1 years. They also discovered that a new equilibrium point could also be obtained $15.6 \%$ closer to the Earth and that the transfer time would reduce to 1.86 years. $^{49}$ Therefore, as a starting point for a transfer from geosynchronous orbit to an equilibrium point ahead of the $\mathrm{L}_{2}$ point, 1.86 years must be assumed initially for this

\footnotetext{
${ }^{47}$ Michiel Otten and Colin R. McInnes, "Near Minimum-Time Trajectories for Solar Sails", Journal of Guidance Control and Dynamics, Vol. 24, No. 3, 2001, p. 634.

${ }^{48}$ Sun H. Hur and Arthur E. Bryson Jr., "Minimum Time Solar Sailing From Geosynchronous Orbit to the Sun-Earth L2 Point", AIAA-92-4657, AAS/AIAA Astrodynamics Conference, August 1992, p. 542.

${ }^{49}$ Ibid p. 542.
} 
thesis. However, as the characteristic acceleration becomes more defined for the geomagnetic tail mission application, the transfer time will be reinvestigated.

\subsection{Solar Sail Mission Applications}

Solar sailing is a very different form of propulsion compared with conventional chemical rockets because there is a continuous thrust applied to the spacecraft from the Sun's photons interacting with the sail. As well, there is no requirement for additional on-board fuel as the spacecraft derives all of its manoeuvring thrust from the solar sail. Therefore, this form of propulsion can perform certain unique missions that could be considered prohibitive for chemical propulsion.

Leipold et al. have written extensively on the concept of a Mercury orbiter that uses a solar sail for propulsion. Due to Mercury's location, use of a chemical rocket to insert a spacecraft into orbit around the planet would be very prohibitive because of the large amount of fuel that is required. In fact, Leipold estimates that to "deliver two identical spacecraft, each of $800 \mathrm{~kg}$ dry mass with a scientific payload of about $100 \mathrm{~kg}$, to highly elliptical orbits about Mercury a total mass of about $6600 \mathrm{~kg}$ for [a] 1997 launch, and about $5900 \mathrm{~kg}$ for [a] 1999 launch, respectively, would be injected by a Titan IV/Centaur launch vehicle in this scenario." ${ }^{90}$ Therefore a ballistic launch could run between $\$ 60$ million and $\$ 120$ million depending upon the launch vehicle.

A solar sail mission, by comparison, could be considerably less expensive due to the reduced weight of the overall spacecraft. Leipold et al. have proposed two different spacecraft sizes for the mission to Mercury: a $500 \mathrm{~kg}$ spacecraft including a $50 \mathrm{~kg}$ 
scientific payload and a $240 \mathrm{~kg}$ spacecraft including a $20 \mathrm{~kg}$ scientific payload. ${ }^{5152}$ Both spacecraft are considerably smaller than the proposed ballistic mission. In related papers a Mercury orbit has been designed in detail showing how solar sail propulsion can be used to maintain a Sun-synchronous polar orbit. ${ }^{53}$ The advantage of a Sun-synchronous orbit is that the spacecraft is not affected by any thermal shock since it would not pass into Mercury's shadow. Leipold et al. have also provided detailed trajectory analysis, a spacecraft conceptual design, details of the scientific payload instruments including a thorough discussion on remote sensing, a basic thermal analysis and launch options. As a mission concept, a Mercury orbiter using solar sail propulsion has been thoroughly investigated and appears to be very viable.

Another mission for a solar sail propelled spacecraft would be a solar polar orbiter. The Ulysses mission, a joint ESANASA project launched in the early 1990 s, is the only solar mission designed to explore the structure of the upper latitudes. Using a gravity assist by Jupiter, it managed to attain a very highly elliptical orbit up to $70^{\circ}$ in latitude. However, a solar sail could provide a circular orbit and up to $90^{\circ}$ latitude. Using a "cranking" orbit, a solar sail can increase its inclination incrementally until a pure polar orbit is attained. Also, the possibility exists with a solar sail to spiral in towards the Sun for a tighter circular orbit, thereby allowing the period of each orbit to be

\footnotetext{
${ }^{50}$ M. Leipold, E. Borg, S. Lingner, A. Pabsch, R. Sachs and W. Seboldt, "Mercury Orbiter with a Solar Sail Spacecraft", Acta Astronautica, Vol. 35, Supplemental, 1995, p. 642.

${ }^{51}$ Ibid, pp. 635-644.

${ }^{52}$ M. Leipold, W. Seboldt, S. Lingner, E. Borg, A. Herrmann, A. Pabsch, O. Wagner and J. Brückner, "Mercury Sun-Synchronous Polar Orbiter with a Solar Sail, Acta Astronautica, Vol. 39, No. 1-4, 1996, pp. 143-151.

${ }^{53}$ Manfred E. Leipold and Otto Wagner, "Mercury Sun-Synchronous Polar Orbits Using Solar Sail Propulsion", Journal of Guidance, Control and Dynamics, Vol. 19, No. 6, Nov-Dec 1996, pp. 1337-1341.
} 
a matter of months rather than years. ${ }^{54}$ McInnes described this smaller orbit in his article entitled "Near-Term, Low Cost Missions for Solar Sails" where "a shorter 3:1 resonance obtained with an orbit radius of $0.48 \mathrm{AU}$ provides good solar imaging while keeping the spacecraft close to $90^{\circ}$ from the Sun-Earth line for much of the mission." ${ }^{955}$ This would allow for the imaging equipment to not only take measurements of the Sun's properties but also monitor for any Coronal Mass Ejections (CMEs) that may be headed towards Earth. McInnes concludes by stating "the mission is truly enabled by solar sailing and makes optimum use of the technology by tackling a high energy mission which is essentially impossible for conventional propulsion."

With CMEs playing a very critical role in the formation of magnetic storms, NASA launched the Advanced Composition Explorer (ACE) spacecraft on 25 August 1997 to the $L_{1}$ equilibrium point to give an early warning in the event of an approaching CME. Since the distance of the $L_{1}$ point is 1.5 million kilometers sunward of the Earth, this gives an indication of an incoming CME roughly one hour ahead of its arrival. By comparison, warning time can be roughly doubled by using solar sail propulsion and positioning a science payload even further forward than the $L_{1}$ point. ${ }^{57}$ A couple of authors have publications describing this kind of solar sail mission application. In Solar Sailing: Technology, Dynamics and Mission Applications, McInnes has detailed a "Geostorm" mission including the orbital dynamics and basic spacecraft design while

\footnotetext{
${ }^{54}$ M. Leipold, "To the Sun and Pluto with Solar Sails and Micro-sciencecraft", Acta Astronautica, Vol. 45, Nos. 4-9, 1999, pp. 549-555.

${ }^{55}$ Colin R. McInnes, "Near-Term, Low Cost Missions for Solar Sails", Journal of the British Interplanetary Society, Vol. 53, No. 1-2, 2000, p. 52.

${ }^{56}$ Ibid, p. 53.

${ }^{57}$ Colin R. McInnes, Solar Sailing: Technology, Dynamics and Mission Applications, Praxis Publishing Ltd., Chichester, UK, 1999, p. 232.
} 
another early warning design has been proposed by Prado et al. from CNES (Centre National d'Études Spatiales) called VIGIWIND. Whereas both designs have the spacecraft operating further Sunward of the $L_{1}$ point, the only difference between them is the solar sail and payload design. Geostorm uses a $130 \mathrm{~kg}$ square solar sail spacecraft with inflatable rigidized diagonal struts while VIGIWIND uses a $160 \mathrm{~kg}$ "rigidized inflatable torus structure which is connected to the bus and payload through four inflatable radial booms." 98 McInnes states that this particular mission application is not demanding from a solar sail technology development aspect and, therefore, could be the best mission by which to launch an inaugural solar sail spacecraft.

As mentioned previously, Forward's "statite" concept described a solar sail propelled spacecraft that seemed to hover above the Earth along an equilibrium contour. In the same article Forward also suggested a "polar observer" mission application due to the fact that "the solar sail thrust would be controlled so that the statite traveled in a slightly elliptical orbit around the Sun with a period equal to the Earth orbital period of one year." ${ }^{, 59}$ Forward considered using the equilibrium contours around the $L_{2}$ point for his concept of a polar observer, however, this may prove to be impractical once the effects of a "real" sail are realized. ${ }^{60}$ In a complimentary study, Dynacon Enterprises Limited (Kieran Carroll et al.) has investigated the possibility of positioning a solar sail propelled spacecraft, called POLARES, high above the Earth along one of the $\mathrm{L}_{1}$

\footnotetext{
${ }^{58}$ Prado, J.Y., Perret, A., Pignolet, G. \& Dandouras, I., "Using a Solar Sail for a Plasma Storm Early Warning System", IAA-96-IAA.3.3.06, 47 International Astronautical Congress, October 1996, p. 7. 59 Robert L. Forward, "Statite: A Spacecraft That Does Not Orbit", Journal of Spacecraft and Rockets, Vol. 28, No. 5, 1991 , p. 609.

${ }^{60}$ Colin R. McInnes, Solar Sailing: Technology, Dynamics and Mission Applications, Praxis Publishing Ltd., Chichester, UK, 1999, p. 253.
} 
equilibrium contours. The POLARES concept includes a $90 \mathrm{~kg}$ communications satellite launched from a Pegasus XL launch vehicle which would be capable of providing communications to the northern polar region. Due to the significant distance from Earth of the equilibrium position ( 2.5 million $\mathrm{km}$ ), voice communication would be impractical but broadcast, data and fax would all be possible. Carroll et al. conclude the study by stating that the POLARES concept is very feasible and would be "highly beneficial for Canada, both in terms of its practical use and the experience gained in a developing field of space technology.

For a final category under solar sail mission applications, several authors have investigated the possibility of using solar sails to propel a spacecraft to the outer solar system and beyond. Since the effect of a solar sail diminishes by an inverse square relationship with distance from the Sun, proceeding with a logarithmic spiral trajectory to any distance beyond the asteroid belt would prove to be impractical. As well, the solar radiation pressure is too weak for braking manoeuvres around the outer planets if solar sailing is the sole propulsion system. However, a different technique to achieve relatively high velocities would be to complete a solar fly-by with a solar sail and thereby use the Sun's photons to their fullest momentum transfer potential.

Leipold and Wagner detailed a mission to Pluto in their article entitled "Solar Photonic Assist Trajectory Design for Solar Sail Missions to the Outer Solar System and Beyond" whereby Pluto can be reached within 13.2 years using a single "solar photonic assist" (solar fly-by) or 10.4 years using a dual solar photonic assist. They also compared

\footnotetext{
${ }^{61}$ Kieran A. Carroll, Peter C. Hughes \& Donald F. Simmons, "Solar Sail Applications, Dynamics and Control Study," Dynacon Report 29-501/01-1, Dynacon Enterprises Ltd., March 1995, p 39.
} 
the calculated solar sail parameters with the "Pluto Express" chemical propulsion concept which would arrive at Pluto in 11.8 years. The solar sail options were very comparable with the "Pluto Express" baseline with the advantage that the solar sail options "could be performed on a low-cost launch vehicle, avoiding also the radiation hazards of a Jupiter gravity assist. ${ }^{962}$

Another intensive study being pursued uses a solar sail to deliver a radio telescope to the focus of the solar gravitational lens. This mission concept is entitled the Aurora Project. Led by Vulpetti and Maccone, the mission uses a high-performance all-metal sail and a unique trajectory characterized by orbital angular momentum reversal capable of achieving a cruise speed in the order of 10 to $20 \mathrm{AU}$ per year. ${ }^{63}$ The goal is to deliver the telescope to the gravitational lens, located at $550 \mathrm{AU}$, within 10 years after launch. Due to this time constraint, the Aurora Project is perhaps the most challenging mission being investigated due to the very advanced technologies required for sail configuration, deployment, sail materials and innovative sail trajectories.

\subsection{Summary}

The concept of solar sailing was originally viewed as a rather fantastic form of propulsion which could normally be found in a science fiction novel. However, with chemical rockets quickly reaching their mission application limits, innovative propulsion concepts are being given very serious thought for rather unique missions. In the past decade alone, interest in solar sailing has increased dramatically such that various

\footnotetext{
${ }^{62}$ M. Leipold and O. Wagner, "Solar Photonic Assist Trajectory Design for Solar Sail Missions to the Outer Solar System and Beyond", AAS/GSFC $13^{\text {th }}$ International Symposium on Space Flight Dynamics, Goddard Space Flight Center, Greenbelt, Maryland, May 11-15, 1998, p. 1041.

${ }^{63}$ Giovanni Vulpetti, “3D High-Speed Escape Heliocentric Trajectories By All-Metallic-Sail Low-Mass Sailcraft", Acta Astronautica, Vol. 39, No. 1-4, pp. 161-170.
} 
international groups are pursuing the development of solar sail technology and all its aspects.

The existing literature on solar sailing is extensive for an "unflown" technology covering such subjects as: orbital dynamics, sail configurations and deployment, trajectory optimization, sail and support structure materials, and mission applications. There is definitely sufficient information on the various aspects of the solar sailing technology to be able to detail the spacecraft specifications required for the mission application envisioned by this thesis. 


\section{Chapter 2 - The Earth's Geomagnetic Environment}

The space environment contains a very complex assortment of ionized plasmas with electromagnetic properties that greatly influence the Earth's space weather. Since the objective of this thesis is to use solar sail propulsion to "park" a scientific payload in the Earth's geomagnetic tail for the purpose of monitoring geomagnetic storm activity, a fundamental understanding of the space environment is required. Therefore, it is important to understand the structure of the solar wind and how it interacts with the Earth's magnetic field so that mission parameters may be determined for spacecraft design criteria.

\subsection{Solar Wind Structure}

The Sun is a fairly "cool" G2V class star which produces a phenomenon called the solar wind. ${ }^{64}$ The solar wind is produced due to the pressure differential between the base of the solar corona and the interstellar medium. This phenomenon can be characterized as a "proton-electron gas that streams past the Earth with a mean velocity of $400-500 \mathrm{~km} / \mathrm{sec} . " 65$ Since the solar wind is an extension of the solar corona and exists out past $150 \mathrm{AU}$, any change in solar activity will be transmitted along it and influence terrestrial magnetospheres.

Another characteristic of the solar wind is the existence of a weak Interplanetary Magnetic Field (IMF). Since the IMF remains attached to the Sun, it forms a spiral shape

\footnotetext{
${ }^{64}$ Tamas 1. Gombosi, Physics of the Space Environment, Cambridge University Press, USA, 1998, p. 211. The Sun is considered a cool star relative to the entire spectrum of stars in the universe.

${ }^{65}$ Thomas F. Tascione, Introduction to the Space Environment, Orbit Book Company, USA, 1988, p. 24.
} 
due to the Sun's orbital rotation of 25 days. The IMF is predominantly within the ecliptic plane "although, at times, [it] can have a substantial component perpendicular to the ecliptic plane." ${ }^{.66}$ The direction of the perpendicular component is indicative of the potential for magnetic storm activity.

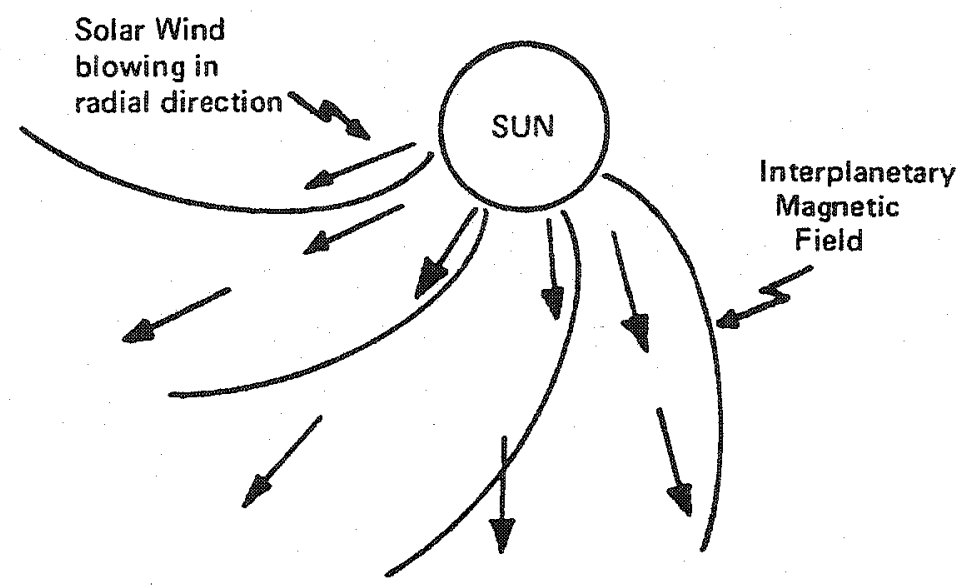

Figure 2.1: Spiral Structure of the Interplanetary Magnetic Field. (From Tascione, 1988.)

The manner in which the solar wind develops a perpendicular component is due to its magnetic structure. Since the solar wind is part of the solar corona, its structure will depend on solar activity. Figure 2.2 shows that the solar wind tends to have uniform sectors of magnetic field direction.

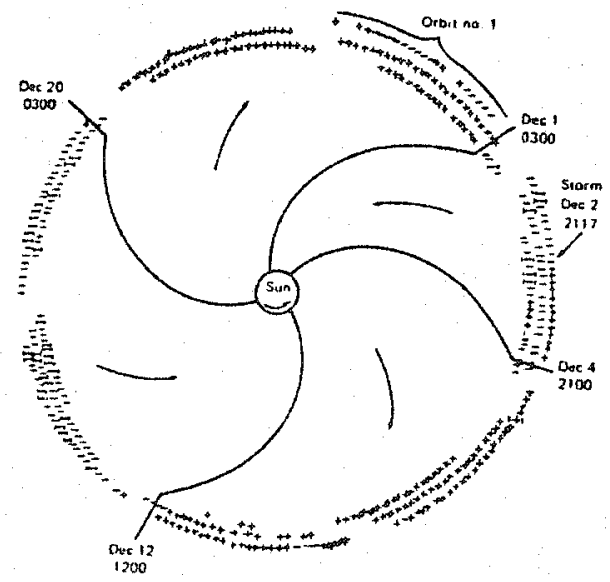

Figure 2.2 Solar magnetic sector structure showing magnetic field direction. (From Tascione, 1988.)

${ }^{66}$ Tascione, p. 26. 
Along with the magnetic sectors, the dipole nature of the solar magnetic field "adds latitudinal structure to the interplanetary medium."67 This means that there is a magnetic field directed away from the Sun in one hemisphere and an opposite one in the other hemisphere. These two fields must be separated by a "current sheet" or "neutral sheet" otherwise the two oppositely charged fields could nullify each other. Since the current sheet is inclined to the solar rotational equator by $7^{\circ}$, this tends to give it a wavy structure as shown in Figure 2.3. ${ }^{68}$

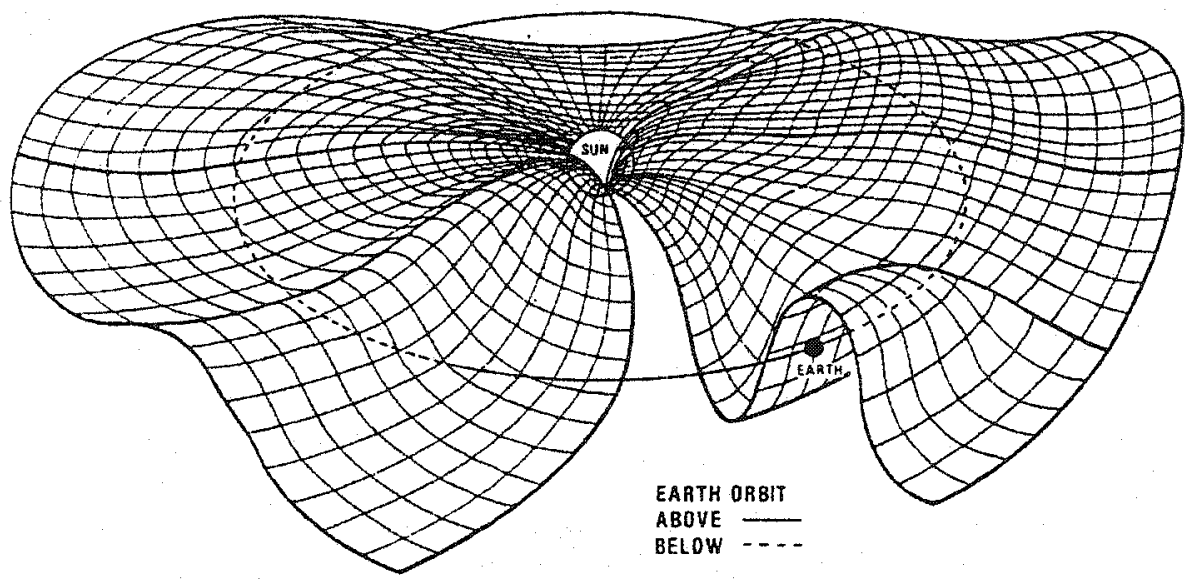

Figure 2.3: Wavy structure of heliomagnetic current sheet. (From Tascione 1988.)

This wavy structure necessitates that Earth will cross the current sheet at least twice per solar rotation. The current sheet crossings are "observed at Earth as crossings of interplanetary magnetic sector boundaries, when the polarity of the interplanetary magnetic field changes its sign." formation of geomagnetic storms and substorms.

\footnotetext{
${ }^{67}$ Tascione, p. 28.

${ }^{68}$ Ibid, p. 28.

${ }^{69}$ Gombosi, p. 251.
} 


\subsection{Earth's Magnetosphere}

The Earth's magnetic field produces a "semipermeable obstacle to the solar wind and the resulting interaction produces a cavity around which most of the plasma flows.,, 70 This obstacle is called the magnetosphere and is illustrated in Figure 2.4.

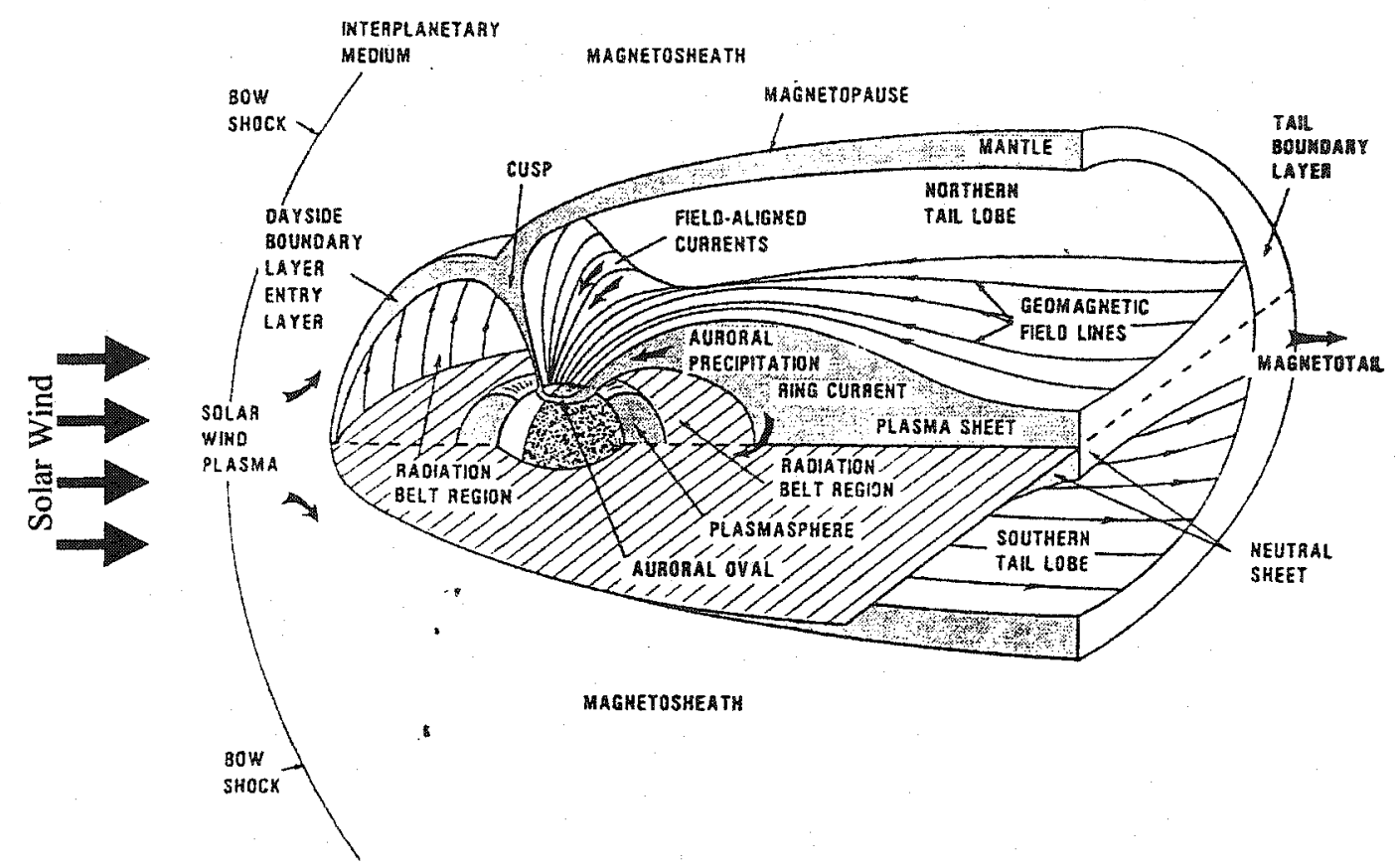

Figure 2.4 Cross section of Earth's magnetosphere. (From Tascione, 1988.)

The magnetosphere is bullet shaped on the sunward side and nearly cylindrical for the tail section. The shape will change depending upon solar activity, solar wind density, solar wind velocity and IMF strength and variation. The magnetopause is the surface where "the outward force of the compressed geomagnetic field (along with the gas pressure of the magnetospheric plasma) is balanced by the force of the solar wind plasma."71 Essentially the majority of the solar wind plasma flows around the magnetosphere and does not get past the magnetopause. Since the solar wind is considered to be supersonic, a bow shock forms in front of the magnetosphere. The area 
in between the bow shock and the magnetopause is an area of rather chaotic magnetic fields and irregularly distributed plasma called the magnetosheath.

The geomagnetic tail is the region that streams behind the Earth away from the Sun. This region is of great importance for the magnetosphere because it acts as a reservoir for plasma and energy. "The energy and plasma are released into the inner magnetosphere aperiodically during magnetically disturbed episodes called magnetospheric substorms." ${ }^{, 72}$ Therefore, the geomagnetic tail would be a very valid observation objective for a scientific payload. The geomagnetic tail also has a current sheet that separates the tail lobes which are regions of oppositely aligned magnetic fields.

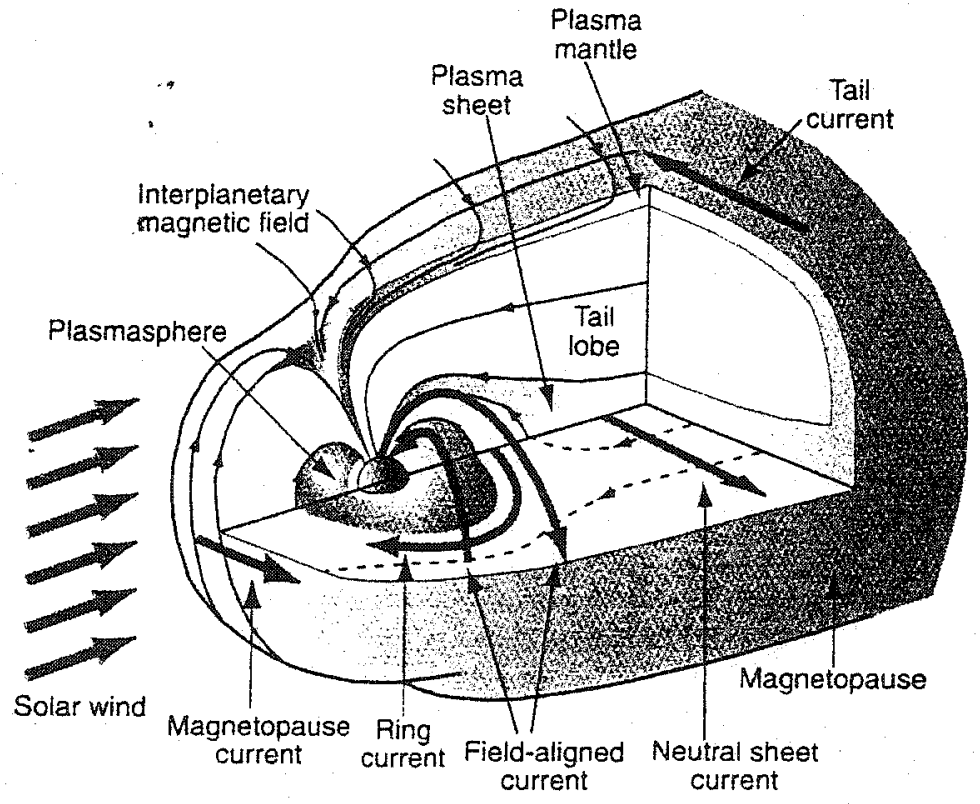

Figure 2.5 Cross section of the magnetosphere showing the various current systems. (From Gombosi, 1998.)

Along with plasma, the magnetosphere has a very complex system of currents which exist and interact with the IMF. Figure 2.5 shows that there are four principle

\footnotetext{
${ }^{70}$ Tascione, p. 45.

${ }^{71}$ Ibid, p. 45.

${ }^{72}$ W.J. Hughes, "The Magnetopause, Magnetotail, and Magnetic Reconnection", Introduction to Space Physics, Kivelson and Russell, Ed., Cambridge University Press, USA, 1995, p. 232.
} 
currents: the magnetopause current, the ring current, the field-aligned current and the neutral sheet current. The interaction of these current systems with the IMF has been a very important research subject for scientists because of how the systems play a critical role in the formation of magnetic storms.

Dimensions for the magnetosphere, and more specifically the geomagnetic tail, are depicted in figure 2.6. As the diagram shows, the front of the magnetopause starts at about $10 R_{E}$ with the bow shock sitting at $13 R_{E}$. The tail itself stretches for a great

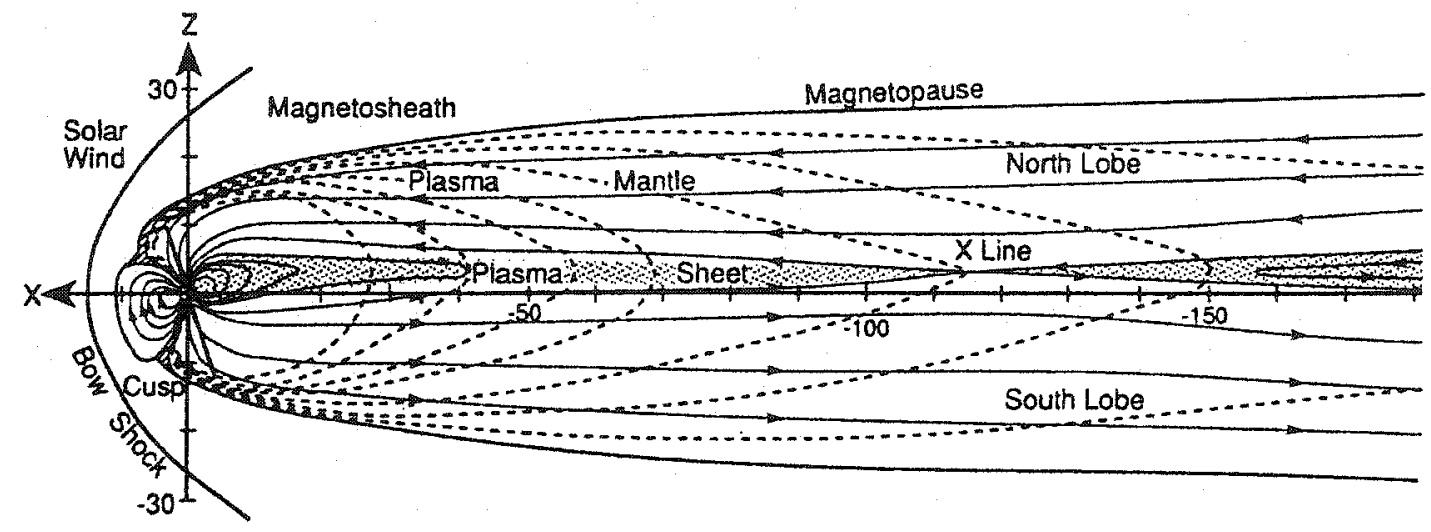

Figure 2.6: Noon-midnight cross-section of magnetosphere and geomagnetic tail with dimensions of Earth Radii $\left(R_{E}\right)$. The moon's orbit is at $60 R_{E}$. (From Kivelson \& Russell, 1995.)

distance behind the Earth, although its limiting distance has yet to be determined by observations. The radius of the magnetosphere starts at $15 \mathrm{R}_{\mathrm{E}}$ just above the poles and "flares" to about $27 R_{E}$ downstream. The flaring of the geomagnetic tail reaches its maximum radius at approximately $140 \mathrm{R}_{\mathrm{E}}{ }^{73}$ For an ultimate length of the geomagnetic tail, one author has suggested $600 \mathrm{R}_{\mathrm{E}}$, although he states "tail field lines that have disconnected from the Earth will retain a tail geometry several thousand $R_{E}$ beyond the Earth., ${ }^{, 74}$ Therefore the true length of the geomagnetic tail has yet to be determined.

\footnotetext{
${ }^{73}$ Ibid, p. 235.

${ }^{74}$ Ibid, p. 245.
} 
One other very important point on the diagram is the magnetic $x$-line which is a neutral point for the plasma or neutral sheet. In this diagram, the $x$-line is depicted at 115 $R_{E}$, however, it may be parabolic in shape such that it could be "as close as $100 R_{E}$ at the center of the tail, but probably is $200 \mathrm{R}_{\mathrm{E}}$ distant at the flanks." ${ }^{.75}$ A phenomenon called magnetic reconnection is believed to be taking place at the $\mathrm{x}$-line and this phenomenon is also associated with magnetic storms and substorms.

\subsection{Geomagnetic Storms and Substorms}

The formation of magnetospheric storms and substorms results from magnetic reconnection of the IMF with the magnetopause current which is attributed to a "southward" IMF component. Figure 2.7 depicts the reconnection process which allows magnetic flux to transfer from the dayside magnetosphere to the geomagnetic tail.

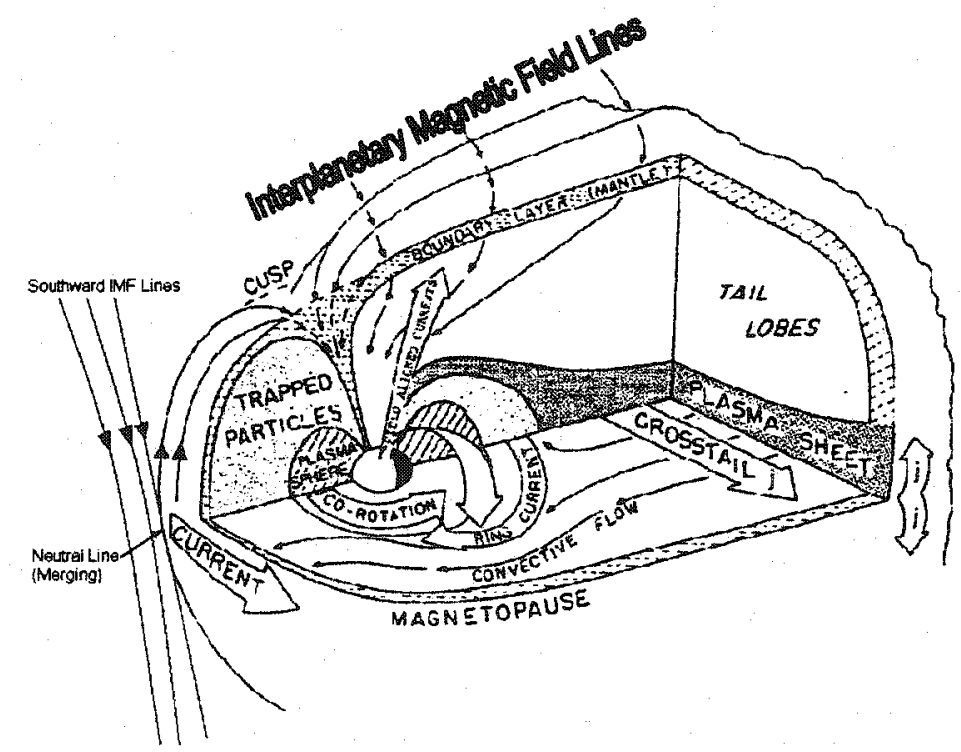

Figure 2.7 Magnetic field topology with neutral line (merging) region depicted for dayside reconnection. (From Tascione, 1988.)

\footnotetext{
${ }^{75}$ R.L. McPherron, "Magnetospheric Dynamics", Introduction to Space Physics, Kivelson and Russell, Ed., Cambridge University Press, USA, 1995, p. 433.
} 
Magnetic storms are "large, prolonged disturbances of the magnetosphere caused by variations in the solar wind," usually following solar flares or coronal mass ejections. ${ }^{76}$ The main requirement for a magnetic storm is a prolonged period of time when the IMF has a significant southward component. Isolated substorms, however, are characterized by "relatively brief (about one hour) periods of southward IMF.",77

The geomagnetic tail performs a critical function during storm development by acting as a reservoir for magnetic flux and plasma. As the magnetic flux increases, "the magnetic field strength in the tail increases in conjunction with the plasma sheet becoming gradually thinner in its north-south extent, shrinking toward the equatorial plane. ${ }^{, 78}$ The plasma or current sheet becomes thinner due to increased pressure in the tail lobes.

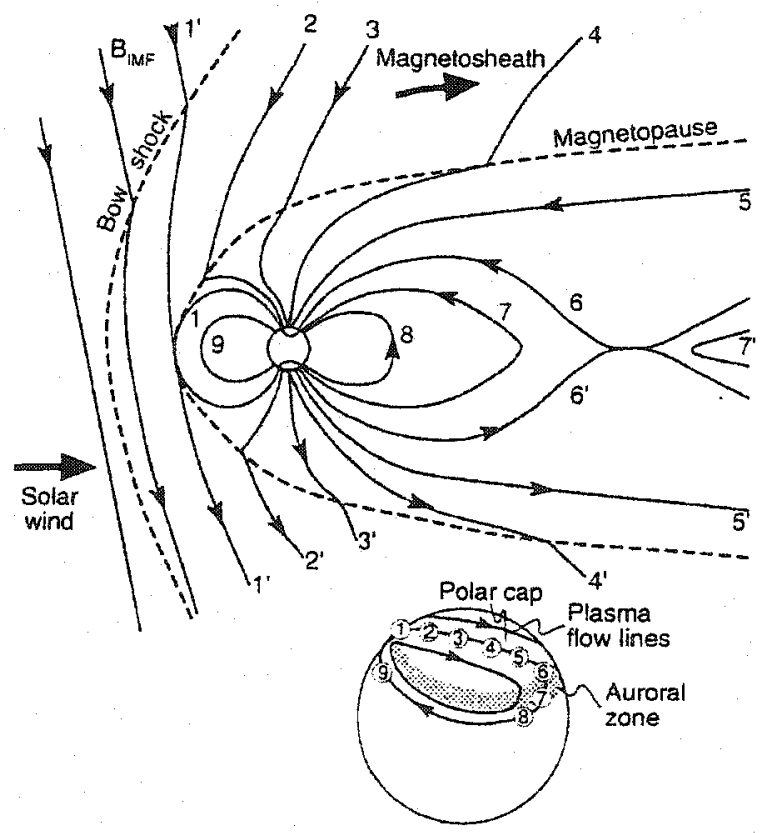

Figure 2.8 Schematic of plasma flow within the magnetosphere driven by magnetic reconnection. Note the southward directed IMF. (From Kivelson \& Russell, 1995.)

\footnotetext{
${ }^{76}$ Gombosi, p. 308.

${ }^{77}$ Ibid, p. 309.

${ }^{78}$ Tascione, p. 55.
} 
Eventually the cross tail current sheet becomes unstable and reconnection occurs in the geomagnetic tail. "This reconnection disrupts the cross-tail current and part of the current is diverted toward the ionosphere along magnetic field lines connecting the reconnection region to the polar region of Earth." $" 79$ It is this mechanism that generates the auroral electrojet which then becomes the physical manifestation of a geomagnetic storm called the aurora borealis.

\subsection{Summary}

The space environment is a complex structure made up of magnetic fields and plasmas interacting with the Earth's intrinsic magnetic field. The magnetosphere is the semipermeable structure which is formed from the solar wind interacting with Earth's magnetic field. The geomagnetic tail forms the portion of the magnetosphere that streams behind the Earth away from the Sun and also performs a significant role in the formation of magnetospheric storms and substorms.

Several authors have described the size and shape of the magnetosphere and, more specifically, the geomagnetic tail. The following table captures those parameters.

\begin{tabular}{|l|c|c|c|}
\hline Geomagnetic Tail Parameter & Earth Radii $\left(\mathrm{R}_{\mathrm{E}}\right)$ & Meters & Astronomical Units $(\mathrm{AU})$ \\
\hline Maximum Radius & 27 & $1.72206 \times 10^{8}$ & 0.00115 \\
X-line - minimum & 100 & $6.378 \times 10^{8}$ & 1.004266 \\
X-line - maximum & 200 & $12.756 \times 10^{8}$ & 1.008532 \\
Tail Flare Limit & 140 & $8.9292 \times 10^{8}$ & 1.00601 \\
Maximum Length & 600 & $38.268 \times 10^{8}$ & 1.0256 \\
\hline
\end{tabular}

Table 2.1: Summary of geomagnetic tail dimensions.

\footnotetext{
${ }^{79}$ Gombosi, p. 310.
} 
Since the objective of this thesis is to launch a solar sail propelled spacecraft into the geomagnetic tail, the parameters specified previously will be used as mission parameters. The design considerations for the solar sail will be based on attempting to position and steer the spacecraft within the geomagnetic tail parameters. In the forthcoming chapters, equations of motion for a solar sail in a restricted three-body system will be developed to determine if the dimensions specified in table 2.1 can be attained. 


\section{Chapter 3 - Solar Sail Propulsion}

The premise behind solar sail propulsion is that light has a pressure associated with it which imparts a momentum to a surface and, that this transfer of momentum results in a force applied to an object. Since a "mirrored" surface would also reflect the light, the total force applied would be a combination of the incident force and the reflected force. If the reflecting surface is considered ideal, meaning that all of the light is reflected and there is neither absorption nor diffusion, then the incident and reflecting forces are equal and the total force is double the incident force. This is the concept behind generating propulsion by use of a solar sail. It must be noted that this issue is not new for spacecraft designers since they have been concerned in the past with solar pressure as a perturbing force for large cross section spacecraft. In fact, some satellite designs actually use small reflecting tabs attached to solar panels to help stabilize the spacecraft with solar radiation pressure.

While the effects of solar radiation pressure on satellites and spacecraft have been known, propulsion systems have continued to primarily be focused on chemical rockets. However, chemical propulsion relies on the use of on-board propellants for traveling to the required orbit as well as station keeping. Once the propellants are expended, the spacecraft is considered useless.

Solar sail spacecraft, by comparison, could remain active for much longer periods of time due to the limitless supply of propulsion from the Sun. Since the velocity change $(\Delta V)$ of a spacecraft is dependent upon the quantity of propellant, a solar sail spacecraft 
would have an extremely high $\Delta V$ due to the inexhaustible radiation energy from the Sun. This high $\Delta V$ means that solar sail propulsion would be able to accomplish certain missions unattainable by chemical propulsion. Also, a savings in mass could be realized since sail structures and material weigh less than chemical propellants and engines. ${ }^{80}$ Any mass savings could mean either an increased payload mass allowed within the same spacecraft mass budget or a cost savings from the launch costs if a smaller and, therefore, cheaper launch vehicle could be used.

\subsection{Solar Radiation Pressure}

The theory of solar radiation pressure was proposed by Maxwell but further investigation into the characteristics of light was completed by Planck and Einstein. Planck proposed "the energy of the [black body thermal] cavity must be quantised at discrete levels," however, he still believed that light propagated as a wave. ${ }^{81}$ Einstein argued that "the radiative energy was itself emitted in discrete packets, preserving its identity as a photon as it propagated. ${ }^{982}$ Therefore, from Planck's law:

$$
E=h v
$$

a photon of light will have energy proportional to its frequency (v) multiplied by Planck's constant $(h)$. Einstein provided an energy equation from his theory of special relativity:

$$
E^{2}=m_{0}^{2} c^{4}+p^{2} c^{2}
$$

\footnotetext{
${ }^{80}$ Kieran A. Carroll, Peter C. Hughes \& Donald F. Simmons, "Solar Sail Applications, Dynamics \& Control Study", Dynacon Report 29-501/01-1, March 1995, p.6.

${ }^{81}$ Colin R. McInnes, Solar Sailing: Technology, Dynamics and Mission Applications, Praxis Publishing Ltd., Chichester, UK, 1999, p. 34. The development of the solar radiation pressure equations is taken directly from this text.

${ }^{82}$ Ibid. p. 34.
} 
where $m_{0}$ is the rest mass of the body, $c$ is the speed of light and $p$ is its momentum. The first term represents the energy of the body at rest while the second term represents the energy of the body in motion. Given that a photon has zero rest mass, (3.2) becomes:

$$
E=p c
$$

Substituting in (3.1) gives:

$$
h v=p c \text { or } p=\frac{h v}{c}
$$

Therefore the momentum transported by a photon of light is proportional to the frequency and inversely proportional to the speed of light.

To determine the solar radiation pressure, the momentum of a flux of photons must now be considered. The energy flux $(W)$ is defined as the energy crossing unit area in unit time and is proportional to the solar luminosity $\left(L_{s}\right)$ and inversely proportional to the square of the distance from the Sun $(r)$. The energy flux at the Earth's distance from the Sun (1 AU) is written as:

$$
W_{E}=\frac{L_{s}}{4 \pi r_{E}^{2}}
$$

and the energy flux at different distances may be written as:

$$
W=W_{E}\left(\frac{r_{E}}{r}\right)^{2}
$$

Using the definition for energy flux, it is possible to write an equation for the change in energy.

$$
\Delta E=W A \Delta t
$$


where $A$ is the area of a surface normal to the incident radiation and $\Delta t$ is the change in time that the energy is transported across the surface. From (3.3), this energy transports a momentum:

$$
\Delta p=\frac{\Delta E}{c}=\frac{W A \Delta t}{c}
$$

Since pressure is defined as the force per unit area and force can be defined as the momentum per unit time:

$$
\begin{gathered}
P=\frac{F}{A} \text { where } F=\frac{\Delta p}{\Delta t} \\
P=\frac{1}{A}\left(\frac{\Delta p}{\Delta t}\right)
\end{gathered}
$$

Then substituting in (3.8) for $\Delta p$ in (3.10) gives:

$$
P=\frac{W}{c}
$$

At the Earth's distance, the energy flux, $W_{E}=1368 \mathrm{~J} / \mathrm{s} \mathrm{m}^{2}$. Therefore, the pressure exerted on a perfectly reflecting solar sail at $1 \mathrm{AU}$ is $\mathrm{P}=4.56 \times 10^{-6} \mathrm{~N} / \mathrm{m}^{2}$ which is considered very small. However, although the solar radiation pressure is small, it is enough to affect a large cross section spacecraft like a solar sail and provide propulsion.

\subsection{Solar Sail Force Model}

To develop some performance metrics for a solar sail it is important to analyze the force exerted on a perfectly reflecting surface. For the development of this force model, the solar sail will be represented by a simple flat surface that reflects all incident light. 
Since the sail can be oriented, the acceleration of the spacecraft is dependent upon the sail's attitude.

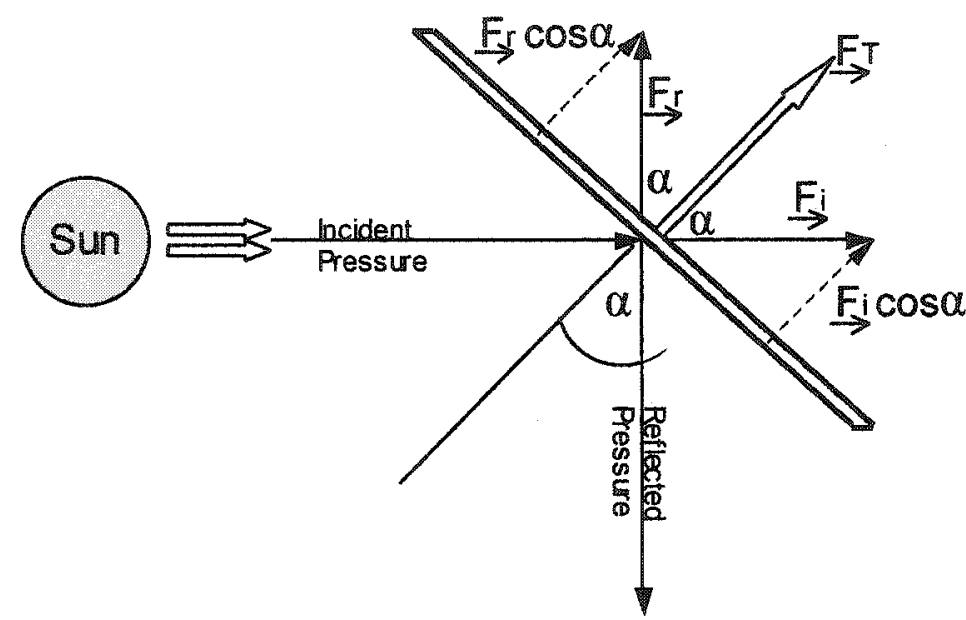

Figure 3.1 Light forces on a reflecting sail.

Figure 3.1 depicts the forces exerted on a perfectly reflecting surface by the Sun's radiation pressure. ${ }^{83}$ The light is reflected through an angle $(\alpha)$ which is measured from the sail normal to the direction of the reflected sunlight. Given that the force is equal to the solar radiation pressure multiplied by the effective area:

$$
F=P A_{e f f}
$$

and $A_{\text {eff }}$ is equal to the total sail area multiplied by the cosine of the angle $(A \cos \alpha)$, then:

$$
F_{i}=P A \cos \alpha
$$

Substituting in (3.11) for $P$ becomes:

$$
F_{i}=\frac{W A}{c} \cos \alpha
$$

\footnotetext{
${ }^{83}$ Robert L. Forward, "Light-Levitated Geostationary Cylindrical Orbits: Correction and Expansion", Journal of the Astronautical Sciences, Vol. 38, No. 3, July-September 1990, pp. 335-338. The development of the force model has been adapted from Forward's work.
} 
Since the sail is assumed to reflect all light perfectly, then:

$$
F_{r}=F_{i}=\frac{W A}{c} \cos \alpha
$$

As depicted in Figure 3.1, the incident and the reflected forces have both normal and transverse components. In fact, the transverse components cancel each other out and the normal components add together to get the total force. Therefore:

$$
F=F_{i} \cos \alpha+F_{r} \cos \alpha
$$

Substituting (3.15) into (3.16) gives:

$$
F=\left(\frac{W A}{c} \cos \alpha\right) \cos \alpha+\left(\frac{W A}{c} \cos \alpha\right) \cos \alpha=\frac{2 W A}{c} \cos ^{2} \alpha
$$

Therefore, the magnitude of the total force that a solar sail can generate is a function of the cosine squared of the "tilt" angle and this force is directed in the sail normal direction.

\subsection{Optimum Sail Angle}

Now that the magnitude and direction of the force have been determined, it is useful to break the force vector down into its radial and transverse components. If the spacecraft is traveling in the $\hat{\jmath}$ direction (figure 3.2), then the transverse force of the simplified planar sail is the component of force used to change the energy state of the spacecraft and, therefore, increase or decrease the orbit depending upon orientation. A brief analysis will show that there is an optimum sail tilt angle to maximize the transverse force. 


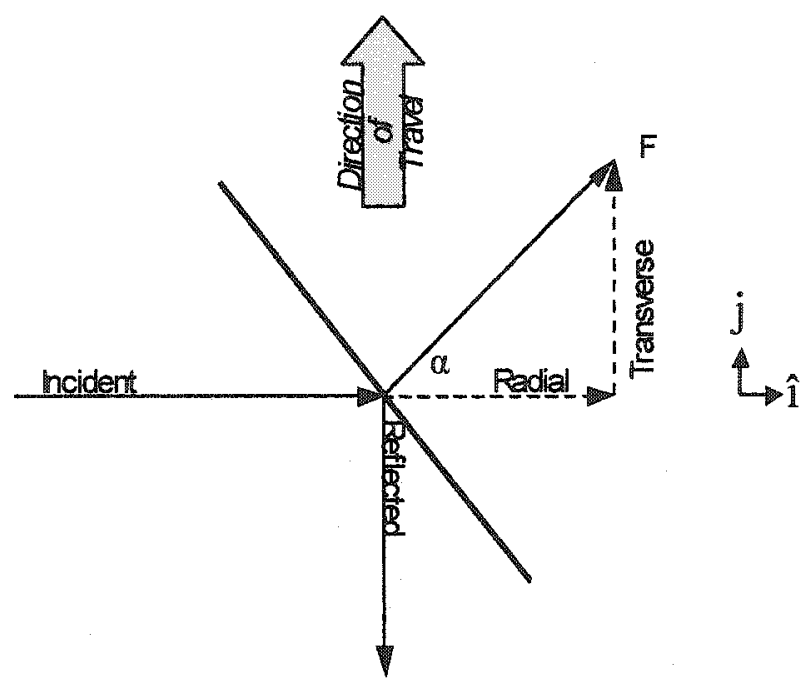

Figure 3.2 Radial and transverse components of solar sail force vector.

Since $F \propto \cos ^{2} \alpha$, it is possible to determine the radial and transverse components of the radiation pressure force from figure 3.2 such that:

$$
F \propto\left(\cos ^{2} \alpha\right)[\cos \alpha \hat{i}+\sin \alpha \hat{j}
$$

Therefore, for the radial component which is in the $\hat{\imath}$ direction:

$$
F_{R} \propto \cos ^{3} \alpha \hat{i}
$$

And the transverse component which is in the $\hat{\jmath}$ direction:

$$
F_{T} \propto \cos ^{2} \alpha \sin \alpha \hat{j}
$$

Now consider that the force has been normalized. To determine the optimum sail angle, take the derivative of the transverse force with respect to $a$ and set it equal to zero.

$$
\begin{gathered}
\frac{d F_{T}}{d \alpha}=2 \cos \alpha(-\sin \alpha) \sin \alpha+\cos ^{2} \alpha \cos \alpha=0 \\
-2 \sin ^{2} \alpha+\cos ^{2} \alpha=0
\end{gathered}
$$

Given that $\cos ^{2} \alpha=1-\sin ^{2} \alpha$,

$$
1-3 \sin ^{2} \alpha=0
$$




$$
\begin{aligned}
& \sin \alpha=\frac{1}{\sqrt{3}} \\
& \alpha=35.26^{\circ}
\end{aligned}
$$

The solar radiation pressure force and its components are depicted in figure 3.3 below.

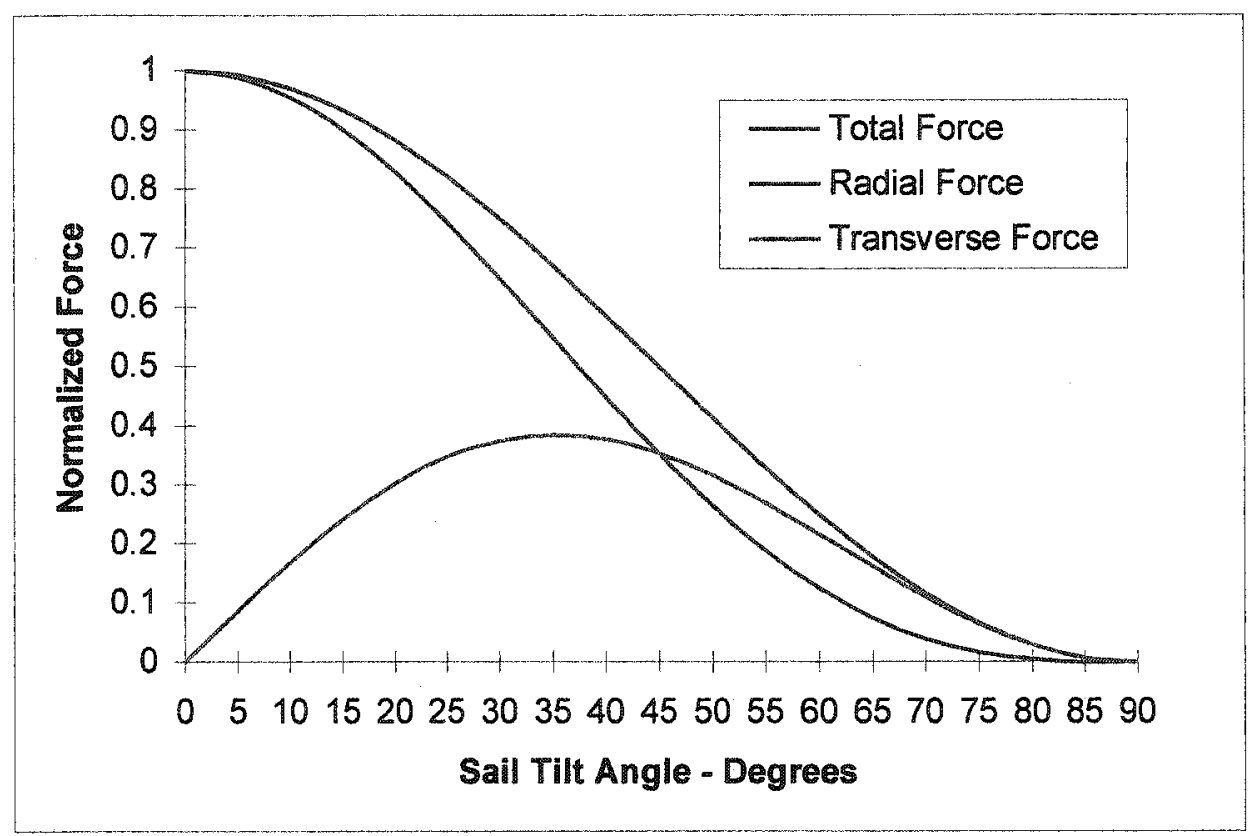

Figure 3.3 Solar sail force components.

While the transverse force component is that which increases the orbital energy in a logarithmic spiral trajectory, it is also the component used to steer the solar sail once on station. If the mission application is to travel within the Earth's geomagnetic tail, a more detailed analysis would be necessary to determine if the sail configuration, proposed in chapter 5 , will be able to steer and control the overall spacecraft effectively. The issue of controlling the spacecraft will be addressed, however, an in-depth analysis is out of scope for this thesis.

\subsection{Performance Metrics}

As the development of solar sailing technologies progressed throughout the last four decades, performance metrics were established to effectively compare various 
aspects of solar sail characteristics. These metrics helped to simplify the unique solar sail orbital dynamics and to demonstrate whether a mission application was even possible.

The first metric is called the sail loading which is represented by $\sigma$ and is simply the mass per unit area of the overall spacecraft.

$$
\sigma=\frac{m}{A} \text { in }\left[\frac{g}{m^{2}}\right]
$$

The second metric is called the characteristic acceleration and is represented by $a_{0}$. The characteristic acceleration is considered the most common metric and is defined as "the solar radiation pressure acceleration experienced by a solar sail facing the Sun at a distance of one astronomical unit (AU)." ${ }^{94}$ Beginning with (3.17) and then substituting $F=m a$ and $A=m / \sigma$ gives:

$$
a=\frac{1}{\sigma} \frac{2 W}{c} \cos ^{2} \alpha
$$

Then by definition, the characteristic acceleration is at $1 \mathrm{AU}$ with $\alpha=0$. It is also possible at this time to introduce the sail efficiency as $\eta$.

$$
a_{0}=\frac{\eta}{\sigma} \frac{2 W}{c}=\frac{9.12 \eta}{\sigma}\left[\frac{m m}{s^{2}}\right]
$$

The efficiency of a sail is dependent upon the type of material used to reflect the sunlight as well as manufacturing accuracy of the sail film and deployments issues such as creases. Given that aluminum has a reflectivity of 0.88 to 0.9 , an efficiency of 0.85

\footnotetext{
${ }^{84}$ Colin R. McInnes, Solar Sailing: Technology, Dynamics and Mission Applications, Praxis Publishing Ltd., Chichester, UK, 1999, p. 13.
} 
may be quite probable. ${ }^{85}$

The third and final metric is called the sail lightness number and is represented by $\beta$. The sail lightness number is considered to be a dimensionless sail loading parameter and is defined as "the ratio of the solar radiation pressure acceleration to the solar gravitational acceleration." Defining $a_{R}$ as acceleration due to radiation pressure and $a_{g}$ as acceleration due to gravity:

$$
\begin{gathered}
a_{R}=\frac{2 W_{E}}{\sigma c}\left(\frac{r_{E}}{r}\right)^{2} \text { where } W_{E}=\frac{L_{s}}{4 \pi r_{E}^{2}} \\
a_{R}=\frac{L_{s}}{2 \pi c r^{2} \sigma}
\end{gathered}
$$

For gravitational acceleration:

$$
a_{g}=\frac{G M_{s}}{r^{2}}
$$

where $G$ is the gravitational constant and $M_{s}$ is the mass of the Sun. Since $\beta$ has been defined as $a_{R} / a_{g}$ :

$$
\begin{gathered}
\beta=\frac{L_{s}}{2 \pi c G M_{s}} \times \frac{1}{\sigma} \\
\beta=\frac{1.529}{\sigma}
\end{gathered}
$$

The significance of the lightness number is that it is dimensionless which means it is a performance characteristic independent of the distance from the Sun. Also, the numerator $\left(1.529 \mathrm{~g} / \mathrm{m}^{2}\right)$ is considered the "critical sail loading", meaning that if a solar

\footnotetext{
${ }^{85} \mathrm{Ibid}, \mathrm{p} .63$.
}

${ }^{86} \mathrm{Ibid}, \mathrm{p} .40$. 
sail could generate a sail loading of $1.529 \mathrm{~g} / \mathrm{m}^{2}$ then $\beta=1$ and the spacecraft would float independent of the Sun's gravitation because the solar radiation pressure acceleration would be equal to the gravitational acceleration. Obviously, if the sail had a sail loading greater than $1.529 \mathrm{~g} / \mathrm{m}^{2}$ then it would actually accelerate away from the Sun. It must be noted that a sail loading of $1.529 \mathrm{~g} / \mathrm{m}^{2}$ 'is an extremely challenging requirement but can enable unique orbits and mission applications for high-performance solar sails. ${ }^{987}$

\subsection{Propulsion Systems Comparison}

Although performance metrics specific to solar sailing have been developed, they cannot be used to compare other types of propulsion systems. Chemical rockets use the specific impulse as defined by the classic rocket equation to compare performance. The definition "states that the specific impulse of a propulsion system is equal to the momentum gained by the rocket per unit weight of propellant consumed." ${ }^{988}$ Since a solar sail does not consume propellant, this definition appears unsuitable. However, by using the rocket equation:

$$
\Delta v=g \times I_{s p} \times \log \left(\frac{m_{0}}{m_{0}-m_{p}}\right)
$$

where $\Delta v$ is the change in speed, $g$ is the gravitational acceleration, $I_{s p}$ is the specific impulse, $m_{0}$ is the rocket's initial mass with propellant, and $m_{p}$ is the propellant mass, it may be possible to compare solar sailing with other propulsion systems. While it could be concluded that solar sails have infinite specific impulse because their mass does not change, during a finite mission duration, the sail will deliver a finite total impulse. In

\footnotetext{
${ }^{87}$ Ibid, p.40.

${ }^{88} \mathrm{Ibid}, \mathrm{p} .17$. The development of the propulsion comparison equations is adapted from this text.
} 
fact, if a solar sail is used to deliver a payload, the "mass of the solar sail itself becomes redundant after the payload is delivered."${ }^{, 89}$

To try to give a representative specific impulse delivered by a solar sail, consider the mass of the propellant in (3.26) to be the mass of a payload carried by a solar sail spacecraft. Rearranging (3.26) will give the effective specific impulse as:

$$
I_{s p}=\frac{\Delta v}{g} \ln \left(\frac{m_{0}}{m_{p}}\right)
$$

where $m_{0}$ is considered the mass of the solar sail and the payload and $m_{p}$ is the mass of the payload only.

The effective change in velocity can also be determined for a solar sail given the characteristic acceleration $\left(a_{0}\right)$ and the mission duration $(T)$ such that $\Delta v \approx a_{0} T$. Therefore (3.27) becomes:

$$
I_{s p} \cong \frac{a_{0} T}{g} \ln \left(\frac{m_{0}}{m_{p}}\right)
$$

Since (3.28) uses the characteristic acceleration, the equation is only accurate close to the Earth. For distances closer to the Sun, the actual acceleration will increase significantly and, therefore, so will the specific impulse. As (3.28) also depicts, the specific impulse increases linearly with mission duration.

This relationship is depicted in figure 3.4 which also compares the specific impulse of a solar sail, that has a payload mass fraction of $1 / 3$, with other propulsion systems.

${ }^{89}$ Ibid, p. 18. 


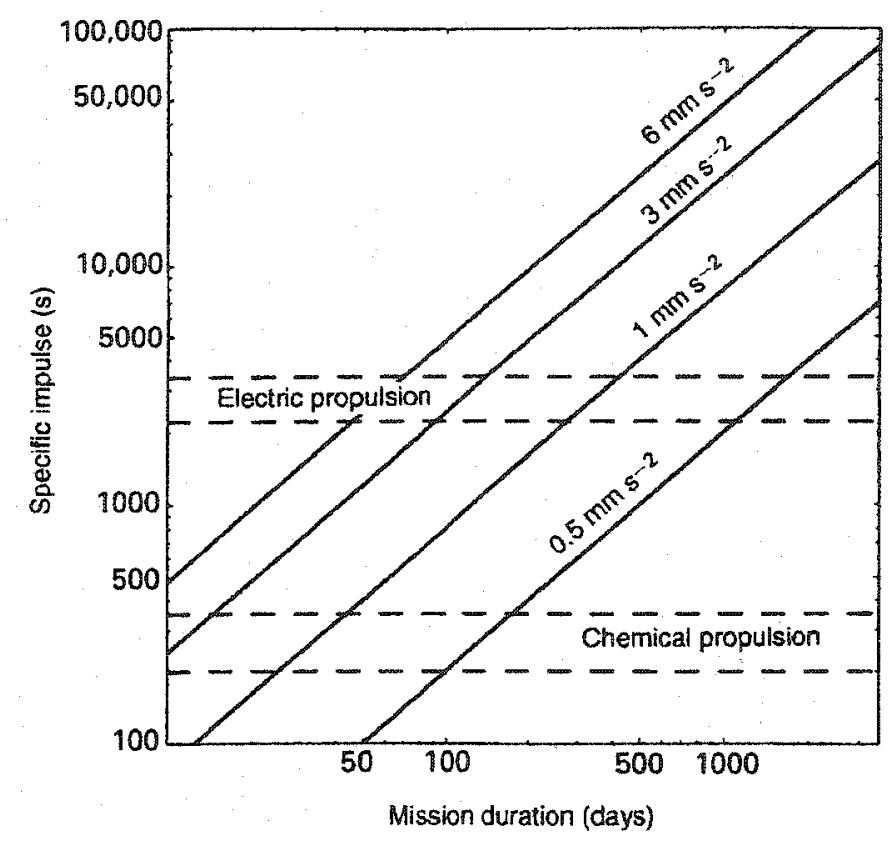

Figure 3.4 Comparison of propulsion systems with solar sail with payload mass fraction of 1/3. (From McInnes, 1999.)

Although very general in nature, figure 3.4 demonstrates that even a modest solar sail with a characteristic acceleration of $0.5 \mathrm{~mm} / \mathrm{s}^{2}$ would be advantageous over chemical propulsion if the mission duration were longer than approximately 200 days. Also, given the requirement for constant thrust to "park" a spacecraft in the Earth's geomagnetic tail, chemical propulsion could become very time limited leaving solar sailing as one of the only alternatives.

\subsection{Summary}

This chapter developed the basic solar sailing force model and performance metrics required to understand the technology. In section 3.5 , it was also demonstrated that solar sailing could be compared with other propulsion systems in relatively general terms. The development of these rudimentary equations lays the foundation for the specific mission application proposed by this thesis. 
In the forthcoming chapter solar sailing in a restricted three-body system will be investigated and equations developed to determine if it is possible to explore the Earth's geomagnetic tail in a parking orbit sunward of the $\mathrm{L}_{2}$ Lagrange equilibrium point. As well, the issue of stability and controllability within this orbit will also be discussed to determine if there will be any problems with station keeping. 


\section{Chapter 4 - Solar Sail Dynamics in a Restricted Three-Body System}

Several authors have developed the equations of motion for a solar sail propelled spacecraft and demonstrated that very unique orbits are possible. While the traditional Keplerian orbits will always exist for a solar sail, McInnes and Forward have shown that non-Keplerian orbits are also possible, since the force generated from the solar radiation pressure can allow the solar sail to "hover" out of plane in a "halo" type of orbit. These novel non-Keplerian orbits could be the vehicle for a new genre of mission applications should solar sailing reach its potential.

The objective of this thesis is to determine if it is possible to place a solar sail spacecraft in the Earth's geomagnetic tail and to manoeuvre within that region. The basis of the orbital dynamics for this mission application is the classic restricted three-body problem. While many great mathematicians worked on this problem and its various aspects, Lagrange showed that there exists five equilibrium points where all the various forces acting on a body would balance each other so that the body could theoretically remain at those specific locations. Figure 4.1 shows these equilibrium points.

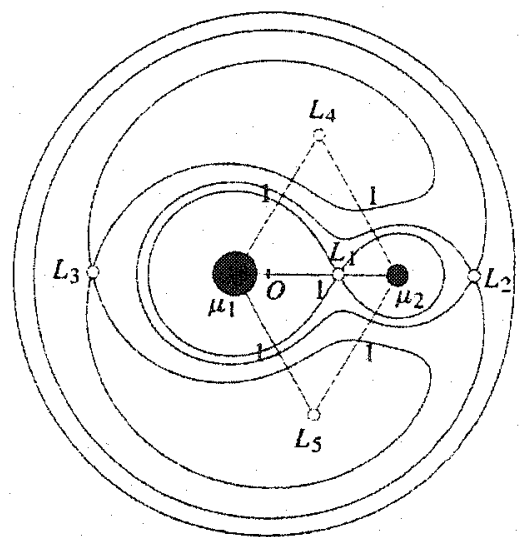

Figure 4.1 Location of the five Lagrange equilibrium points. (From Murray and Dermott, 1999.) 
Since the geomagnetic tail streams anti-sunward behind the Earth, the $\mathrm{L}_{2}$ equilibrium point could be an ideal position for observing the interactions inside the tail. However, as previously mentioned in chapter 2 , the $\mathrm{L}_{2}$ equilibrium point is but one position along the geomagnetic tail and, therefore, would have limited value as an observation position. A better scenario is to be able to travel sunward of the $L_{2}$ point and even out of plane to observe the interactions of the geomagnetic tail. McInnes has demonstrated that this may be possible since the additional thrust generated from solar sailing creates equilibrium contour lines originating from the $L_{2}$ point.

This chapter will determine the location of the Lagrange equilibrium points, develop the equations of motion for a solar sail spacecraft specifically around the $\mathrm{L}_{2}$ point, and then discuss the stability and controllability along the equilibrium contours. Finally, the mission objectives will be applied to determine the solar sail performance metrics required to observe the geomagnetic tail.

\subsection{The Lagrange Equilibrium Points}

In a three-body system, "consider the motion of a small particle of negligible mass moving under the gravitational influence of two masses $m_{l}$ and $m_{2} .900$ There are several assumptions which must be made to simplify the system. First, the masses have circular orbits with a common center of mass. Second, the masses exert a force on the particle but the particle is too small to influence the other two masses. Finally, there are two sets of coordinates for the system: a set of axes in the inertial frame $(\xi, \eta, \zeta)$, and a set of axes in the rotating frame $(x, y, z)$. These coordinates are depicted in Figure 4.2.

\footnotetext{
${ }^{90}$ C.D. Murray and S. F. Dermott, Solar System Dynamics, Cambridge University Press, USA, 1999, p. 64. The development of the Lagrange Equilibrium points is taken directly from this text.
} 


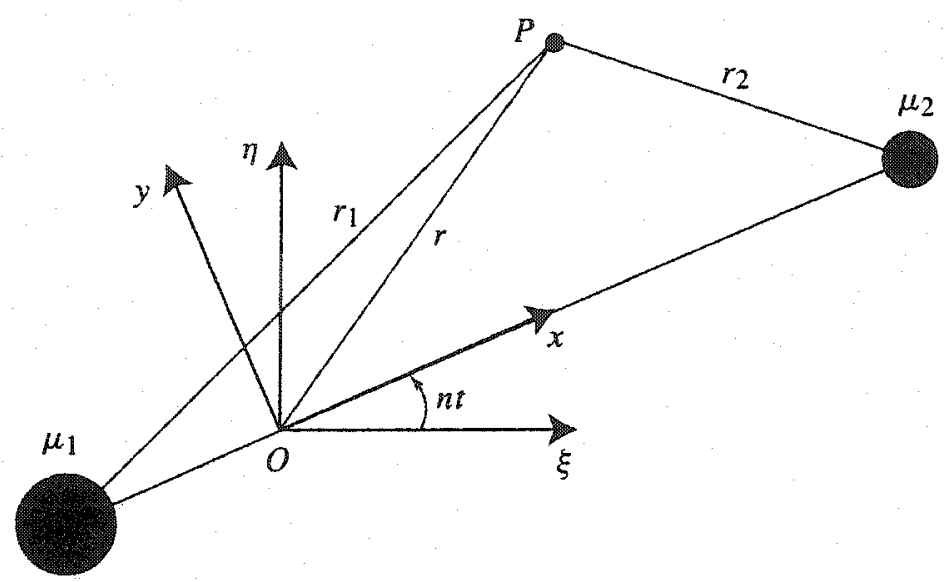

Figure 4.2. Restricted three-body problem set up showing inertial coordinates and rotating coordinates. (From Murray \& Dermott, 1999.)

Let the $\xi$ axis lie along the line from $m_{1}$ to $m_{2}$ at time $t=0$. The $\eta$ axis is perpendicular to the $\xi$ axis in the orbital plane of the two masses and the $\zeta$ axis is perpendicular to the $\xi-\eta$ plane along the angular momentum vector.

Let $\mu_{1}=G m_{1}$ and $\mu_{2}=G m_{2}$ where $G$ is the gravitational constant. Also, the mass ratio would be: $\mu=\frac{m_{2}}{m_{1}+m_{2}}$. To simplify the system even further, consider the sum of the two masses to be unity and the separation between them also unity. Therefore, because $m_{1}+m_{2}=1$, then $\mu_{1}=1-\mu$ and $\mu_{2}=\mu$. Also, the distance from $m_{1}$ to the center of mass $(0)$ would be $\mu$, and the distance from the center of mass to $m_{2}$ would simply be $1-\mu$.

Beginning with the inertial coordinate system and applying the vector form of the inverse square law, the equations of motion of the particle become:

$$
\begin{aligned}
& \ddot{\xi}=\mu_{1} \frac{\xi_{1}-\xi}{r_{1}^{3}}+\mu_{2} \frac{\xi_{2}-\xi}{r_{2}^{3}} \\
& \ddot{\eta}=\mu_{1} \frac{\eta_{1}-\eta}{r_{1}^{3}}+\mu_{2} \frac{\eta_{2}-\eta}{r_{2}^{3}} \\
& \ddot{\zeta}=\mu_{1} \frac{\zeta_{1}-\zeta}{r_{1}^{3}}+\mu_{2} \frac{\zeta_{2}-\zeta}{r_{2}^{3}}
\end{aligned}
$$


Consider also that the two masses are moving about the common center of mass at a fixed angular velocity $n$. Now consider the system from the rotating reference frame which is rotating at the uniform rate $n$ in the positive direction. As depicted in figure 4.1, the rotating frame coordinates have the same origin as the inertial frame. The rotating frame coordinates are related to the coordinates in the inertial system by the rotation:

$$
\left(\begin{array}{l}
\xi \\
\eta \\
\zeta
\end{array}\right)=\left(\begin{array}{ccc}
\cos n t & -\sin n t & 0 \\
\sin n t & \cos n t & 0 \\
0 & 0 & 1
\end{array}\right)\left(\begin{array}{l}
x \\
y \\
z
\end{array}\right)
$$

If each component in (4.4) is differentiated:

$$
\left(\begin{array}{c}
\dot{\xi} \\
\dot{\eta} \\
\dot{\zeta}
\end{array}\right)=\left(\begin{array}{ccc}
\cos n t & -\sin n t & 0 \\
\sin n t & \cos n t & 0 \\
0 & 0 & 1
\end{array}\right)\left(\begin{array}{c}
\dot{x}-n y \\
\dot{y}+n x \\
\dot{z}
\end{array}\right)
$$

and

$$
\left(\begin{array}{c}
\ddot{\xi} \\
\ddot{\eta} \\
\ddot{\zeta}
\end{array}\right)=\left(\begin{array}{ccc}
\cos n t & -\sin n t & 0 \\
\sin n t & \cos n t & 0 \\
0 & 0 & 1
\end{array}\right)\left(\begin{array}{c}
\ddot{x}-2 n \dot{y}-n^{2} x \\
\ddot{y}+2 n \dot{x}-n^{2} y \\
\ddot{z}
\end{array}\right)
$$

By substituting the above equations into (4.1), (4.2) and (4.3), the equations of motion in the rotating frame become:

$$
\begin{gathered}
\ddot{x}-2 n \dot{y}-n^{2} x=-\left[\mu_{1} \frac{x+\mu_{2}}{r_{1}^{3}}+\mu_{2} \frac{x-\mu_{1}}{r_{2}^{3}}\right], \\
\ddot{y}+2 n \dot{x}-n^{2} y=-\left[\frac{\mu_{1}}{r_{1}^{3}}+\frac{\mu_{2}}{r_{2}^{3}}\right] y, \\
\ddot{z}=-\left[\frac{\mu_{1}}{r_{1}^{3}}+\frac{\mu_{2}}{r_{2}^{3}}\right] z
\end{gathered}
$$


These equations can also be written as the gradient of a scalar function $U$ :

$$
\begin{gathered}
\ddot{x}-2 n \dot{y}=\frac{\partial U}{\partial x}, \\
\ddot{y}+2 n \dot{x}=\frac{\partial U}{\partial y}, \\
\ddot{z}=\frac{\partial U}{\partial z},
\end{gathered}
$$

where the function $U$ is given by:

$$
U=\frac{n^{2}}{2}\left(x^{2}+y^{2}\right)+\frac{\mu_{1}}{r_{1}}+\frac{\mu_{2}}{r_{2}} .
$$

It is important to note from (4.13) that the $x^{2}+y^{2}$ term is representative of centrifugal force while the $\mu_{1} / r_{1}$ and $\mu_{2} / r_{2}$ terms represent the gravitational forces exerted on the particle from the two masses.

To determine the locations of the equilibrium points, the equations of motion must be analyzed to determine if there are any points where the "particle has zero velocity and zero acceleration in the rotating frame."91 From figure 4.1 it is possible to write equations for $r_{1}$ and $r_{2}$. The direction of the $x$ axis is such that the two masses always lie along it with coordinates $\left(x_{1}, y_{1}, z_{1}\right)=\left(-\mu_{2}, 0,0\right)$ and $\left(x_{2}, y_{2}, z_{2}\right)=\left(\mu_{1}, 0,0\right)$. Hence:

$$
\begin{aligned}
& r_{1}^{2}=\left(x+\mu_{2}\right)^{2}+y^{2}+z^{2}, \\
& r_{2}^{2}=\left(x-\mu_{1}\right)^{2}+y^{2}+z^{2} .
\end{aligned}
$$

From (4.14) (4.15) and given that $\mu_{1}+\mu_{2}=1$, which is the definition for the unit mass, it

${ }^{91}$ Ibid, p. 77. 
is possible to write:

$$
\mu_{1} r_{1}^{2}+\mu_{2} r_{2}^{2}=x^{2}+y^{2}+\mu_{1} \mu_{2},
$$

and, therefore, it is possible to rewrite (4.13) in a different form as:

$$
U=\mu_{1}\left(\frac{1}{r_{1}}+\frac{r_{1}^{2}}{2}\right)+\mu_{2}\left(\frac{1}{r_{2}}+\frac{r_{2}^{2}}{2}\right)-\frac{1}{2} \mu_{1} \mu_{2} .
$$

Since the equilibrium positions are points where there is neither acceleration nor velocity of the particle, then $\ddot{x}=\ddot{y}=\dot{x}=\dot{y}=0$ so that (4.10) and (4.11) become:

$$
\begin{aligned}
& \frac{\partial U}{\partial x}=\frac{\partial U}{\partial r_{1}} \frac{\partial r_{1}}{\partial x}+\frac{\partial U}{\partial r_{2}} \frac{\partial r_{2}}{\partial x}=0 \\
& \frac{\partial U}{\partial y}=\frac{\partial U}{\partial r_{1}} \frac{\partial r_{1}}{\partial y}+\frac{\partial U}{\partial r_{2}} \frac{\partial r_{2}}{\partial y}=0
\end{aligned}
$$

By solving the partial derivative equations above using (4.17), it is possible to determine the equations for the location of the equilibrium points. After some reduction, the solution takes the form:

$$
\begin{gathered}
\mu_{1}\left(-\frac{1}{r_{1}^{2}}+r_{1}\right) \frac{x+\mu_{2}}{r_{1}}+\mu_{2}\left(-\frac{1}{r_{2}^{2}}+r_{2}\right) \frac{x-\mu_{1}}{r_{2}}=0, \\
\mu_{1}\left(-\frac{1}{r_{1}^{2}}+r_{1}\right) \frac{y}{r_{1}}+\mu_{2}\left(-\frac{1}{r_{2}^{2}}+r_{2}\right) \frac{y}{r_{2}}=0 .
\end{gathered}
$$

From these two equations, the locations of the five equilibrium points may be determined. Murray and Dermott explain in Solar System Dynamics in great detail how the solutions are derived from (4.20) and (4.21), however, it is intended here to only summarize their findings for the five points.

For the $L_{4}$ and $L_{5}$ points, the following solutions determine their position:

$$
x=\frac{1}{2}-\mu_{2} \text { and } y= \pm \frac{\sqrt{3}}{2} \text {. }
$$


Since $r_{1}=r_{2}=1$, each of the two points defined by the above equations forms an equilateral triangle with the two masses.

For the $\mathrm{L}_{1}$ point, the solution for the location becomes:

$$
\begin{gathered}
r_{2}=\alpha-\frac{1}{3} \alpha^{2}-\frac{1}{9} \alpha^{3}-\frac{23}{81} \alpha^{4}+\ldots \ldots . \\
\text { where } \alpha=\left(\frac{\mu_{2}}{3 \mu_{1}}\right)^{1 / 3}
\end{gathered}
$$

For the $L_{2}$ point:

$$
r_{2}=\alpha+\frac{1}{3} \alpha^{2}-\frac{1}{9} \alpha^{3}-\frac{31}{81} \alpha^{4}+\ldots \ldots
$$

Finally, for the $L_{3}$ point:

$$
r_{1}=1-\frac{7}{12}\left(\frac{\mu_{2}}{\mu_{1}}\right)+\frac{7}{12}\left(\frac{\mu_{2}}{\mu_{1}}\right)^{2}-\frac{13223}{20736}\left(\frac{\mu_{2}}{\mu_{1}}\right)^{3}+\ldots .
$$

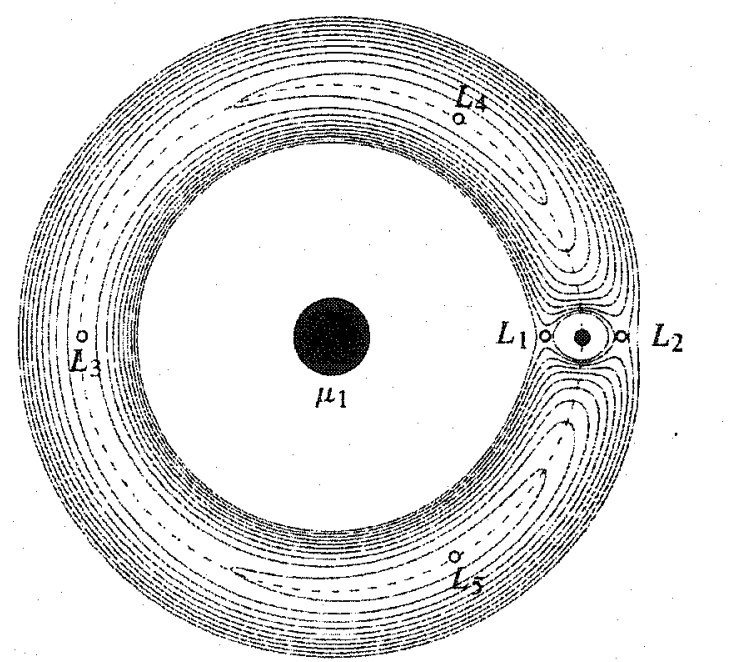

Figure 4.3. The location of the Lagrange equilibrium points for a mass ratio of 0.01 . The dashed line denotes the circle of unit radius centered on the mass $\mu_{1}$. (From Murray \& Dermott, 1999.)

Figure 4.3 depicts the location of the equilibrium points for a system with a mass ratio of 0.01 . Although this mass ratio is much larger than the Earth-Sun system, which is $3.003 \times 10^{-6}$, the diagram gives a pictorial representation of the location of the 
equilibrium points for such a system. Using the Earth-Sun mass ratio and (4.24), the location of the $\mathrm{L}_{2}$ equilibrium point can be calculated and is, therefore, located at $1.0100369 \mathrm{AU}$

\subsection{Solar Sails in Restricted Three-Body Systems}

With the Lagrange equilibrium points now determined, it is possible to adapt solar sailing to this problem. It is important to note that the sail attitude may be oriented in any direction and, therefore, equilibrium solutions will be possible in all three dimensions. Also, since the magnitude of the solar radiation pressure is reflected in the various performance metrics, multiple equilibrium solutions will also be possible. "Therefore, since these parameters can be selected, it is clear that rich new possibilities will arise for artificial equilibrium points. 992

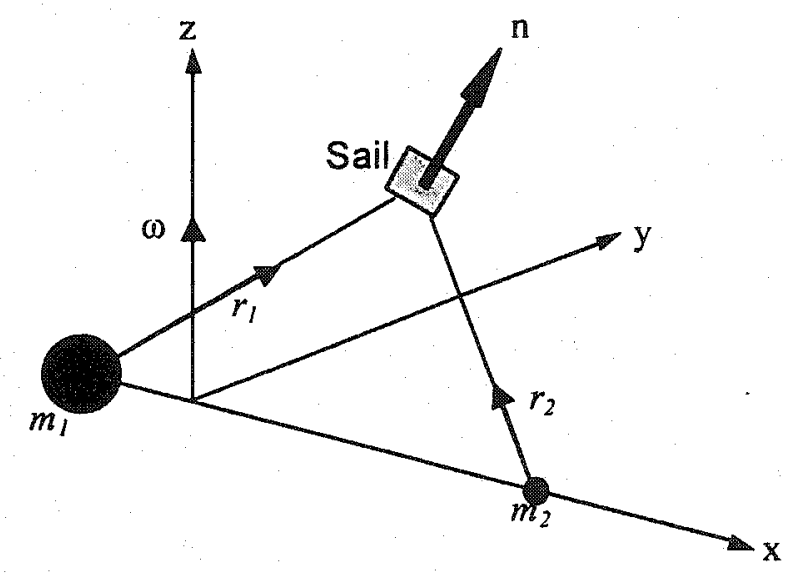

Figure 4.4 Vector representation of a solar sail in a restricted three-body problem. (From McInnes, 1999.)

The geometry of the restricted three-body problem, as depicted in figure 4.4 , is identical to the previous section with the exception of the angular velocity represented by $\omega$. The motion of the two masses about the center of mass is defined by the rotating 
frame of reference $(x, y, z)$. Again the distance between the primary masses and the sum of the masses will be unity to simplify the problem. McInnes has chosen to express the solar sail performance metrics in terms of the sail lightness number $\beta$, however, it is possible to show the equilibrium solutions using any of the other solar sail parameters.

The angular velocity of the reference frame is defined as $\omega=1$ so that the frame co-rotates with the primary masses. Also, the sail attitude is defined by the unit vector $\mathbf{n}$ which is fixed in the rotating frame of reference. Since the sail attitude is fixed to the rotating frame of reference, then the solar sail must rotate about the $z$ axis in time $2 \pi / \omega .{ }^{93}$ This means that as the three-body system rotates, the sail will continue to maintain its attitude with the Sun $\left(m_{l}\right)$ due to the solar radiation pressure and, therefore, there is no requirement to actively steer the sail to maintain this attitude.

The vector equation of motion for a solar sail in this rotating frame of reference may now be written as:

$$
\frac{d^{2} \mathbf{r}}{d t^{2}}+2 \omega \times \frac{d \mathbf{r}}{d t}+\omega \times(\omega \times \mathbf{r})=\mathbf{a}-\nabla V
$$

where the solar radiation pressure acceleration a and the gravitational potential $V$ are defined by:

$$
\begin{gathered}
\mathbf{a}=\beta \frac{1-\mu}{\mathrm{r}_{1}^{2}}\left(\hat{\mathrm{r}_{1}} \cdot \mathbf{n}\right)^{2} \mathbf{n}, \\
V=-\left(\frac{1-\mu}{r_{1}}+\frac{\mu}{r_{2}}\right) .
\end{gathered}
$$

${ }^{92}$ Colin R. McInnes, Solar Sailing: Technology, Dynamics and Mission Applications, Praxis Publishing, Chichester, UK, 1999, p. 214. The development of this problem is taken directly from this text.

${ }^{93}$ Ibid. p. 215 
The equation of motion used by McInnes is simply a different form of the equations developed in the previous section, with the addition of the solar radiation pressure acceleration. The first term of the equation represents the radial acceleration, the second term is the acceleration due to the Coriolis force and the third term is the centrifugal acceleration. As mentioned previously, for equilibrium solutions, the radial and Coriolis force accelerations would equal zero. Therefore, the solution to this problem involves a balance of forces between the solar radiation pressure force, the centrifugal force and the gravitational forces of the two masses on the solar sail.

Through his analysis, McInnes has provided solutions for the sail attitude and the sail lightness number required for equilibrium in the rotating frame of reference. The sail attitude is described in terms of two angles: the cone angle and the clock angle. The sail lightness number is of the form:

$$
\beta=\frac{r_{1}^{2}}{1-\mu} \frac{\nabla U \cdot \mathbf{n}}{\left(\hat{\left.\mathbf{r}_{1} \cdot \mathbf{n}\right)^{2}},\right.}
$$

where $U$ is the same as (4.13) but of a slightly different form. ${ }^{94}$

The solutions provided by McInnes produce regions where equilibrium solutions exist and other regions where they cannot exist. Figure 4.4 depicts the regions and geometry of the equilibrium contours for the region around the Earth $\left(m_{2}\right)$.

${ }^{94}$ Ibid, p. 217 

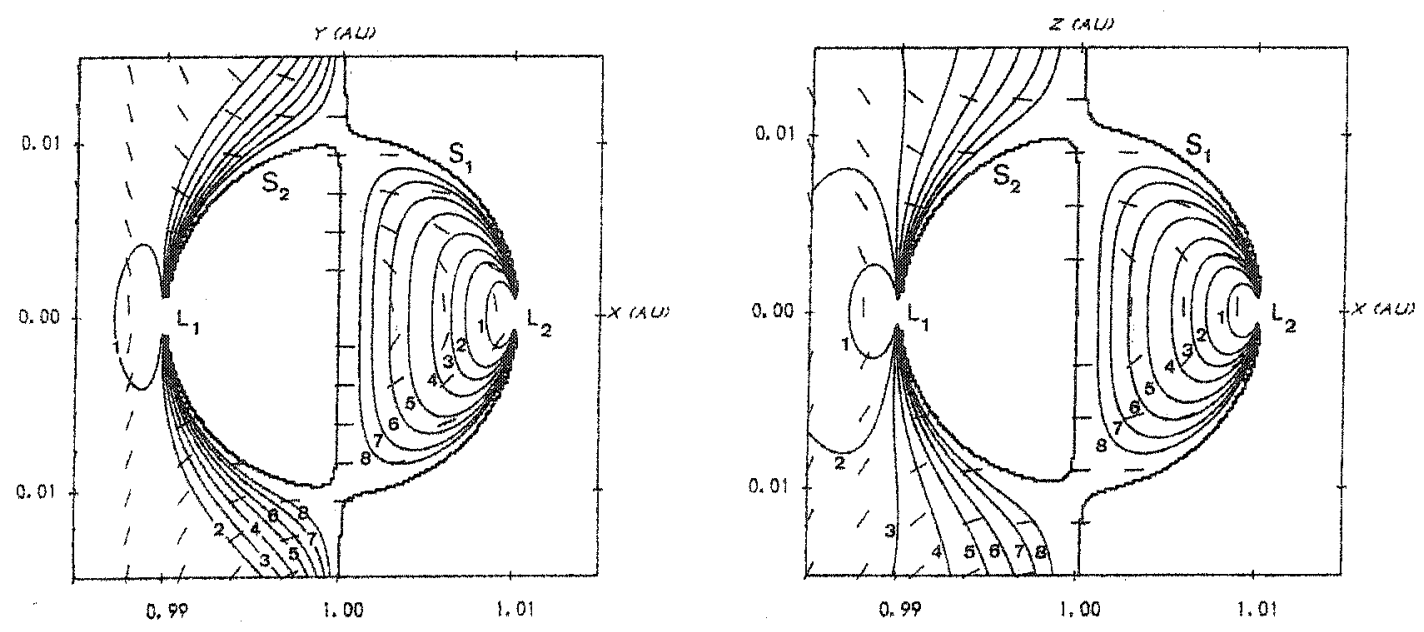

Figure 4.5 Equilibrium contours around the Earth's $\mathrm{L}_{1}$ and $\mathrm{L}_{2}$ points in the $\mathrm{xy}$ and $\mathrm{x}-\mathrm{z}$ planes. (From McInnes, 1999.)

The contours in figure 4.5 are numbered and the respective performance metrics are listed in the table below.

\begin{tabular}{|c|c|c|c|c|c|c|c|}
\hline Contour & 1 & 2 & 3 & 4 & 5 & 6 & 7 \\
\hline$\beta$ & 0.02 & 0.04 & 0.06 & 0.1 & 0.2 & 0.4 & 1.0 \\
$\mathrm{a}_{0}\left(\mathrm{~mm} / \mathrm{s}^{2}\right)$ & 0.12 & 0.24 & 0.36 & 0.59 & 1.19 & 2.37 & 5.93 \\
$\sigma\left(\mathrm{g} / \mathrm{m}^{2}\right)$ & 76.50 & 38.25 & 25.50 & 15.30 & 7.65 & 3.83 & 1.53 \\
\hline
\end{tabular}

Table 4.1 Performance metrics for equilibrium solutions in the vicinity of the Earth. (From McInnes, 1999.)

There are some very important aspects about figure 4.5 which must be noted.

First, the equilibrium solutions are confined to two specific regions, which are defined by the surfaces $S_{1}$ and $S_{2}$ These regions are sunward of the $L_{1}$ point and between the Earth and the $L_{2}$ point. Also, the dash marks represent the rough orientation of the solar sail along the contours. In addition, the performance of a solar sail must increase the further away from the Lagrange equilibrium point that the spacecraft travels so as to maintain 
equilibrium. In theory, a solar sail equipped spacecraft should be able to position itself along these equilibrium contours, including out of plane positions, and maintain equilibrium. Finally, "as the sail lightness number increases, larger volumes of space are accessible for artificial equilibrium points. ${ }^{.95}$

As a rough estimate for the proposed mission application to the geomagnetic tail, a solar sail would be required to travel between the Earth and the $\mathrm{L}_{2}$ point at around contour 4. However, a more precise calculation will be carried out in section 4.4.

\subsection{Equilibrium Solutions for a Non-Ideal Solar Sail}

Now that the equilibrium solutions for an ideal sail have been investigated, it would be prudent to consider the performance of a sail that does not reflect all sunlight perfectly. Since the substance that possesses the best characteristics for a solar sail material is aluminum coated Kapton, then the reflectivity efficiency of aluminum is already known. Obviously any inefficiencies of the sail material will serve to reduce the overall acceleration of the spacecraft, but McInnes has proposed that they may also limit the range of travel along the equilibrium contours.

McInnes has used vector analysis to analyze a sail that is not ideal. To keep the solution of a closed-form nature, "the solar sail will be assumed to have perfect specular reflectivity and no thermal re-emission but will still have an overall reflectivity $\eta$ less than unity. ${ }^{.96}$

\footnotetext{
${ }^{95}$ Colin R. McInnes, "Artificial Lagrange Points for a Partially Reflecting Flat Solar Sail", Journal of Guidance, Control and Dynamics, Vol. 22, No. 1, 1999, p. 186.

${ }^{96}$ Ibid.
} 


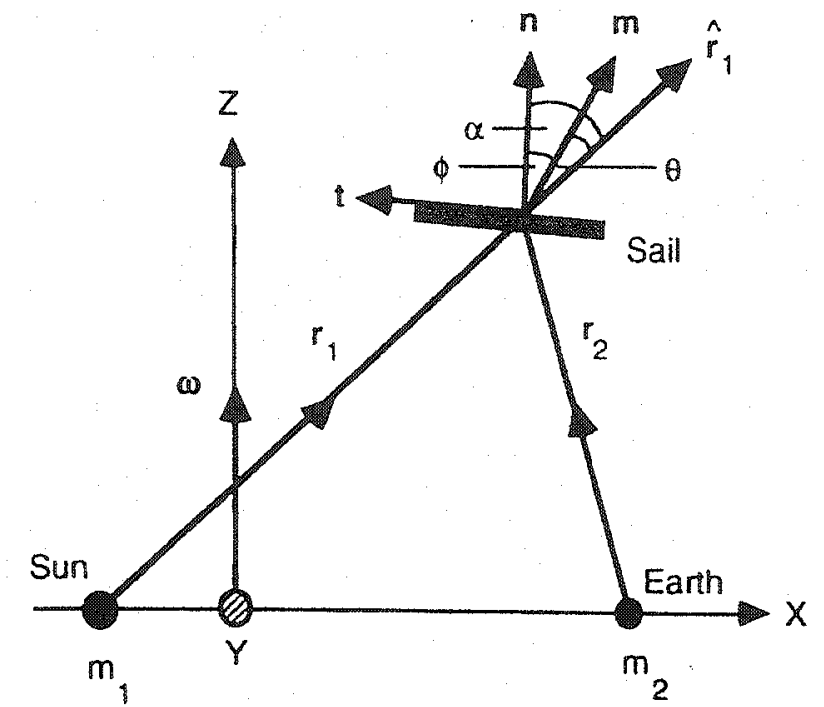

Figure 4.6 Sun-Earth restricted three-body problem with a partially reflecting solar sail. (From McInnes, 1998.)

The solar radiation pressure acceleration due to reflectivity will act in the direction of the sail normal $\mathbf{n}$, while the acceleration due to absorption will act in the direction of the incident force from the sunlight $\mathbf{r}_{1}$. As depicted in figure 4.6, the summation of these two vectors produces an offset vector $\mathbf{m}$ which is the direction in which the total radiation pressure will act. McInnes has shown that the "main effect of the non-perfect reflectivity of the sail is to reduce the acceleration magnitude and to introduce an offset in the direction of the radiation pressure acceleration." ${ }^{.97}$ The angle $\phi$ is called the centerline angle and is that which defines the offset vector, while $\theta$ is defined as the sail cone angle.

Using the same method of vector analysis as for an ideal sail, McInnes has provided a couple of key equations for a non-perfect reflecting solar sail. The first is an equation for the centerline angle calculated directly from the cone angle of the form:

${ }^{97}$ Ibid. 


$$
\tan \phi=\frac{\eta}{(1+\eta) \tan \theta}\left[1-\left(1-\frac{1-\eta^{2}}{\eta^{2}} \tan ^{2} \theta\right)^{\frac{1}{2}}\right]
$$

The second equation is for the sail lightness number $\beta$ in terms of the lightness number for an ideal sail $\tilde{\beta}$ :

$$
\beta=\frac{2}{1+\eta} \frac{\sqrt{1+\tan ^{2} \phi}}{(1-\tan \theta \tan \phi)},
$$

where $\tilde{\beta}$ is derived from (4.29). The results of these equations are depicted below.

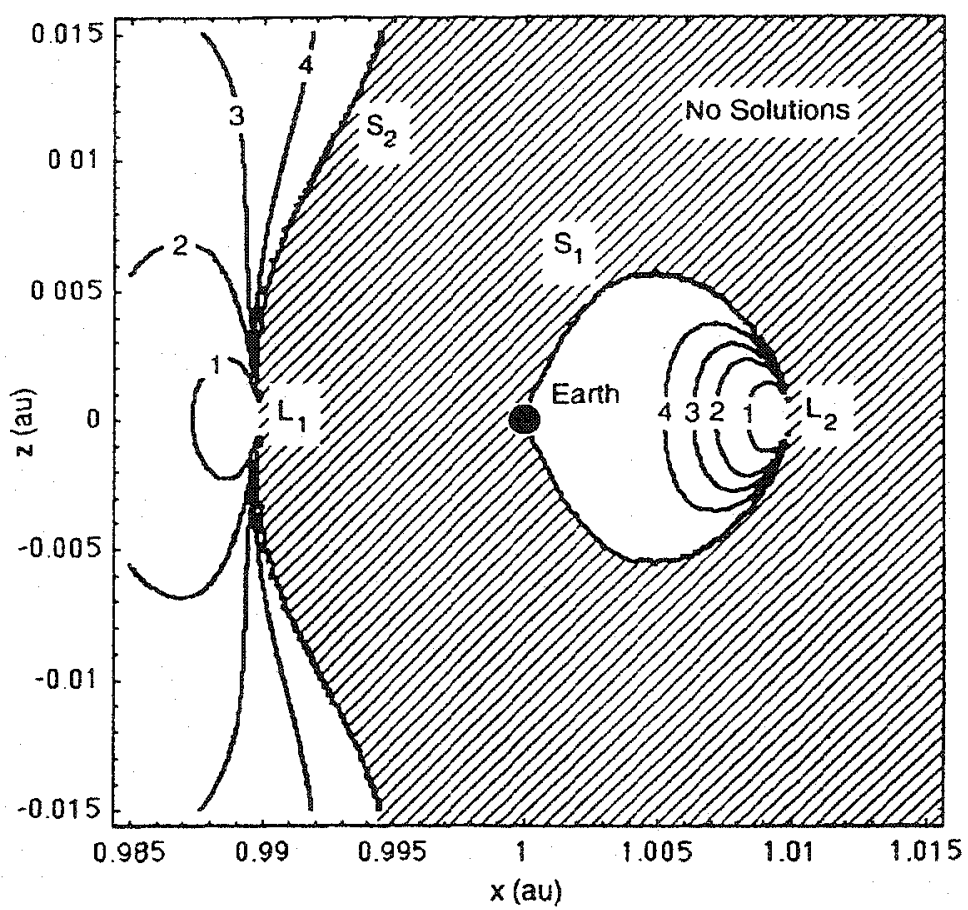

Figure 4.7 Equilibrium Contours of sail lightness numbers in the $x z$ plane with $\eta=0.9$. (From Mclnnes, 1998.)

The most significant effect of a non-ideal solar sail is the reduction in volume near $L_{2}$ of space available for equilibrium solutions. This reduction in space is not anticipated to have a significant impact on the proposed mission application to the Earth's geomagnetic tail, since the out of plane travel will be relatively limited (approximately $30 R_{E}$ ). 


\subsection{Stability and Controllability}

Now that the existence of equilibrium contours have been described, the stability of these contours will be discussed to determine if they are either stable or unstable. If an object is placed in a "stable" region, then it will "remain in the vicinity despite small displacements." ${ }^{" 98}$ Obviously an object placed in an "unstable" region would move away from the equilibrium point or contour and additional propulsion would be required to steer it back into position. While a stable environment would be an ideal solution, a solar sail produces continuous thrust so that controlling the spacecraft position in an unstable region may not be problematic.

The method for examining the stability of a system is by "linearising the equations of motion and carrying out a linear stability analysis. ${ }^{, 99}$ Details of this process are described by Murray \& Dermott in Solar System Dynamics but, for the purposes of this chapter, the main points will be summarized. Referring to (4.10) and (4.11), the equations of motion are modified to include a small displacement $(\mathrm{X}, \mathrm{Y})$ from an equilibrium point denoted by $\left(x_{0}, y_{0}\right)$. After some manipulation and with $n=1$, the result is a set of linear differential equations of the form:

$$
\ddot{X}+2 \dot{Y}=X U_{x x}+Y U_{x y} \text { and } \ddot{Y}+2 \dot{X}=X U_{x y}+Y U_{y y},
$$

where the following quantities are all constants:

$$
U_{x x}=\left(\frac{\partial^{2} U}{\partial x^{2}}\right)_{0}, U_{x y}=\left(\frac{\partial^{2} U}{\partial x \partial y}\right)_{0}, U_{y y}=\left(\frac{\partial^{2} U}{\partial y^{2}}\right)_{0} .
$$

\footnotetext{
${ }^{98}$ C.D. Murray and S.F. Dermott, Solar System Dynamics, Cambridge University Press, USA, 1999, p. 83.

${ }^{99}$ Ibid. p. 85. The development of the stability of equilibrium points is taken directly from this text.
} 
It is possible to write the equations in a matrix form which will help to determine the stability of the system:

$$
\left(\begin{array}{c}
\dot{X} \\
\dot{Y} \\
\ddot{X} \\
\ddot{Y}
\end{array}\right)=\left(\begin{array}{cccc}
0 & 0 & 1 & 0 \\
0 & 0 & 0 & 1 \\
U_{x x} & U_{x y} & 0 & 2 \\
U_{x y} & U_{y y} & -2 & 0
\end{array}\right)\left(\begin{array}{c}
X \\
Y \\
\dot{X} \\
\dot{Y}
\end{array}\right)
$$

The equations are now simplified into four first-order differential equations of the form $\dot{X}=\mathbf{A X}$ where $\mathbf{A}$ is an $n \times n$ matrix of constants and $\mathbf{X}$ is a vector. From theory, if any vector $\mathbf{x}$ satisfies the equation:

$$
A x=\lambda x
$$

where $\lambda$ is a scalar constant, then $\mathbf{x}$ is the eigenvector of the matrix $\mathbf{A}$ and $\lambda$ is the corresponding eigenvalue. It is now possible to rewrite (4.35) of the form:

$$
(\mathbf{A}-\lambda \mathbf{I}) \mathrm{x}=0
$$

where $\mathbb{I}$ is the $n \times n$ unit matrix. The criterion for being able to solve the simultaneous linear differential equations so that the solutions are non-trivial is that the determinant of (4.36) be equal to zero. Therefore,

$$
\operatorname{det}(\mathbf{A}-\lambda \mathbf{I})=0
$$

This will produce the "characteristic equation" which will determine the stability or instability of the system. After further steps and definitions, it is possible to write (4.37) in a form which will produce the characteristic equation:

$$
\operatorname{det}(\mathbf{A}-\lambda \mathbb{I})=\left|\begin{array}{cccc}
-\lambda & 0 & 1 & 0 \\
0 & -\lambda & 0 & 1 \\
U_{x x} & U_{x y} & -\lambda & 2 \\
U_{x y} & U_{y y} & -2 & -\lambda
\end{array}\right|=0
$$


which reduces to the polynomial equation:

$$
\lambda^{4}+\left(4-U_{x x}-U_{y y}\right) \lambda^{2}+U_{x x} U_{y y}-U_{x y}^{2}=0 .
$$

The general solution of the eigenvalues from (4.39) takes the form:

$$
\lambda_{1,2}= \pm\left(j_{1}+\mathrm{i} k_{1}\right), \quad \lambda_{3,4}= \pm\left(j_{2}+\mathrm{i} k_{2}\right),
$$

where $j_{l}, k_{1}, j_{2}$ and $k_{2}$ are real quantities and $i=\sqrt{-1}$ meaning that each eigenvalue could have both a real and/or an imaginary part. If $j=0$, then the eigenvalues would have sin and cosine terms which means that the solution would be oscillatory and, therefore, stable. If $j$ is positive, however, then the eigenvalues would have an exponential growth component which means a non-oscillatory solution and, therefore, an unstable system. Essentially, for a stable point, all eigenvalues must be purely imaginary. For the Lagrange equilibrium points, the triangular points $\left(\mathrm{L}_{4}, \mathrm{~L}_{5}\right)$ are stable with eigenvalues being purely imaginary while the collinear points $\left(\mathrm{L}_{1}, \mathrm{~L}_{2}, \mathrm{~L}_{3}\right)$ are unstable with the eigenvalues being both real and imaginary.

McInnes et al., using the same methodology described above, have conducted an analysis of the stability of the equilibrium contours. ${ }^{100}$. The problem is broken down into two types of stability analysis: asymptotic stability and Lyapunov stability. For the case of asymptotic stability, "it is required that all of the system eigenvalues [be] in the lefthand complex plane." ${ }^{\prime 101}$ Lyapunov stability is that which was described previously in that all of the eigenvalues must be purely imaginary for the system to be stable.

\footnotetext{
${ }^{100}$ McInnes, C. R., McDonald, A. J. C., Simmons, J. F. L., MacDonald, E. W., "Solar Sail Parking in Restricted Three-Body Systems," Journal of Guidance, Control and Dynamics, Vol. 17, No. 2, 1994, pp. 402-403.

101 Ibid. p.402.
} 
McInnes et al. show that solar sailing in a restricted three-body system will produce a characteristic polynomial equation with six roots of the form:

$$
P_{(s)}=\sum_{j=0}^{6} a_{6-j} s^{j}
$$

where the coefficients of the polynomial $a$ were also calculated showing that $a_{1}=0$. Given the fact that $a_{1}=0$, "an application of the Routh-Hurwitz criterion implies that at least one eigenvalue will not lie in the left-hand complex plane." 102 Therefore, since all eigenvalues do not lie in the left-hand complex plane, the system is not asymptotically stable.

To determine whether the system is stable in the Lyapunov sense, McInnes et al. analyzed the eigenvalues to determine whether they were all purely imaginary. They found that stability might be possible but only under certain restrictive conditions. "The required conditions for Lyapunov-type stability are that [the cone angle] $\alpha=0$ or [the sail lightness number] $\beta=0 . " 103$ The first condition means that the solar sail would be oriented along the sun line which means that any other orientation would produce an unstable environment. The second condition would mean the removal of the solar radiation pressure acceleration which is not the intention of the proposed mission application to the geomagnetic tail. Therefore, for practical purposes, the system is considered to be unstable both asymptotically and from the Lyupanov sense.

Although solar sailing in a restricted three-body system does not have a natural stability, it is important to note that the sail produces continuous propulsion which can be used to effectively control the spacecraft. McInnes et al. have outlined a control scheme ${ }^{102}$ Ibid. 
using "proportional and derivative feedback to the sail attitude." 104 An analysis was performed on the controllability of the system with feedback and it was discovered that the system is fully controllable. Also, feedback control allows the inclusion of "gain constants" that can be designed such that all eigenvalues will be in the left-hand complex plane and, therefore, the system will be asymptotically stable.

\subsection{Mission Application}

For the proposed mission application to the Earth's geomagnetic tail, the distances listed in table 2.1 will be used to calculate the performance metrics required for a solar sail to manoeuvre in the Sun-Earth system. Once the performance metrics have been produced for an ideal sail, the effects of a real sail will be included to determine an overall sail loading. Figure 4.8 represents a solar sail manoeuvring in a restricted threebody system with a mass ratio of:

$$
\mu=\frac{m_{2}}{m_{1}+m_{2}}
$$

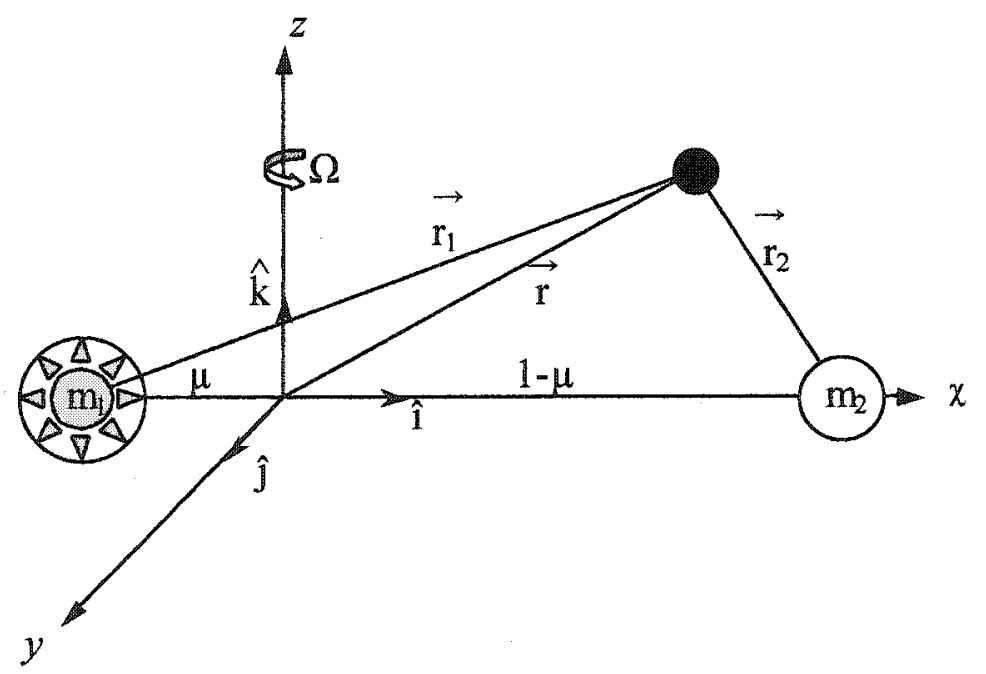

Figure 4.8 Restricted three-body setup for a solar sail in the Sun-Earth system.

103 Tbid. p. 403 .

104 Ibid. 
For simplification, the sum of the mass and the sum of the distances are equal to unit. Therefore, for an equilibrium solution of the system, a balance of forces approach may be used such that:

$$
m_{s} \frac{d^{2} \vec{r}}{d t^{2}}+2 m_{s}\left(\vec{\Omega} \times \frac{d \vec{r}}{d t}\right)+m_{s}(\vec{\Omega} \times(\vec{\Omega} \times \vec{r}))=-\frac{G m_{1} m_{s} \vec{r}_{1}}{\left|r_{1}\right|^{3}}-\frac{G m_{2} m_{s} \vec{r}_{2}}{\left|r_{2}\right|^{3}}+\vec{a}_{s s}
$$

On the left-hand side of the equation the first term represents the radial force, the second term the Coriolis force and the third the centrifugal force. On the right hand side the first two terms refer to the gravitational force from the Sun and the Earth respectively while the final term represents the solar sail acceleration required for equilibrium.

Given that the spacecraft mass $m_{s}$ is significantly smaller than that of the Sun or Earth, it can be neglected. Also, since the spacecraft will be stationary in the Sun-Earth reference coordinates, then the time-dependent components would equal zero. Therefore, the equation for the solar sail acceleration reduces to:

$$
\vec{a}_{s s}=\vec{\Omega} \times(\vec{\Omega} \times \vec{r})+\frac{G m_{1} \vec{r}_{1}}{\left|r_{1}\right|^{3}}+\frac{G m_{2} \vec{r}_{2}}{\left|r_{2}\right|^{3}}
$$

To analyze (4.44) further, each vector entity will be broken down into Cartesian coordinates so that the total acceleration can be calculated. Beginning with the centrifugal acceleration, the angular velocity of the system acts in the $\hat{\mathrm{k}}$ direction and the vector $\vec{r}$ can be represented in Cartesian coordinates as $\vec{r}=x \hat{i}+y \hat{j}+z \hat{\mathrm{k}}$ such that:

$$
\vec{\Omega}=\left(\begin{array}{l}
0 \\
0 \\
\Omega
\end{array}\right)=\Omega \hat{\mathrm{k}} \text { where }|\Omega|=2 \pi \text { radians/year. }
$$


Therefore, the cross product of the angular velocity with the vector $\vec{r}$ is:

$$
\begin{gathered}
\vec{\Omega} \times \vec{r}=\Omega x \hat{j}-\Omega y \hat{i} \text {, and } \\
\vec{\Omega} \times(\vec{\Omega} \times \vec{r})=-\Omega^{2}(x \hat{i}+y \hat{j}) .
\end{gathered}
$$

Note that there is no component in the $\hat{\mathrm{k}}$ direction so that the angular acceleration is a projection in the $x-y$ plane.

For the gravitational acceleration terms, it is important to define the vectors $\vec{r}_{1}$ and $\vec{r}_{2}$ in terms of Cartesian coordinates. From figure 4.7 it can be determined that:

$$
\begin{aligned}
& \vec{r}_{1}=(x+\mu) \hat{\mathrm{i}}+\hat{y} \hat{\mathrm{j}}+z \hat{\mathrm{k}} \text {, and } \\
& \bar{r}_{2}=(x-(1-\mu) \hat{\mathrm{i}}+y \hat{\mathrm{j}}+z \hat{\mathrm{k}}
\end{aligned}
$$

Therefore, given that $\left|r_{1}\right|=\sqrt{(x+\mu)^{2}+y^{2}+z^{2}}$, then the gravitational acceleration due to the Sun is:

$$
\frac{G m_{1} \vec{r}_{1}}{\left|r_{1}\right|^{3}}=\frac{G m_{1}}{\left[(x+\mu)^{2}+y^{2}+z^{2}\right]^{3 / 2}}\left(\begin{array}{c}
x+\mu \\
y \\
z
\end{array}\right)
$$

For the gravitational acceleration due to the Earth:

$$
\frac{G m_{2} \vec{r}_{2}}{\left|r_{2}\right|^{3}}=\frac{G m_{2}}{\left[(x-(1-\mu))^{2}+y^{2}+z^{2}\right]^{3 / 2}}\left(\begin{array}{c}
x-(1-\mu) \\
y \\
z
\end{array}\right) \text {. }
$$

Therefore, the total acceleration required for the solar sail to maintain equilibrium is:

$\vec{a}_{s s}=-\Omega^{2}\left(\begin{array}{l}x \\ y \\ 0\end{array}\right)+\frac{G m_{1}}{\left[(x+\mu)^{2}+y^{2}+z^{2}\right]^{3 / 2}}\left(\begin{array}{c}x+\mu \\ y \\ z\end{array}\right)+\frac{G m_{2}}{\left[(x-(1-\mu))^{2}+y^{2}+z^{2}\right]^{3 / 2}}\left(\begin{array}{c}x-(1-\mu) \\ y \\ z\end{array}\right)$ 
It is now possible to calculate the required acceleration for the solar sail using the distances listed in table 2.1. Using the minimum $x$-line distance of $100 R_{E}$ as a desired position along the $x$ axis from which to observe the geomagnetic tail and the maximum tail radius of $27 \mathrm{R}_{\mathrm{E}},(4.52)$ can now be solved. As a point of clarification, $x$ represents the distance from the Sun-Earth barycenter to the solar sail. Therefore, $x+\mu$ is the distance from the Sun to the solar sail position along the $x$ axis and $x-(1-\mu)$ is the distance from the Earth to the solar sail position.

For the case where the solar sail is at the desired minimum distance of $100 R_{E}$ on the $x$ axis but does not manoeuvre out of plane, (4.52) reduces to:

$$
\begin{gathered}
a_{s s}=\left[-\Omega^{2} x+\frac{G m_{1}}{(x+\mu)^{2}}+\frac{G m_{2}}{(x-(1-\mu))^{2}}\right] \hat{\mathrm{i}}, \\
a_{s s}=0.9039 \mathrm{~mm} / \mathrm{s}^{2} .
\end{gathered}
$$

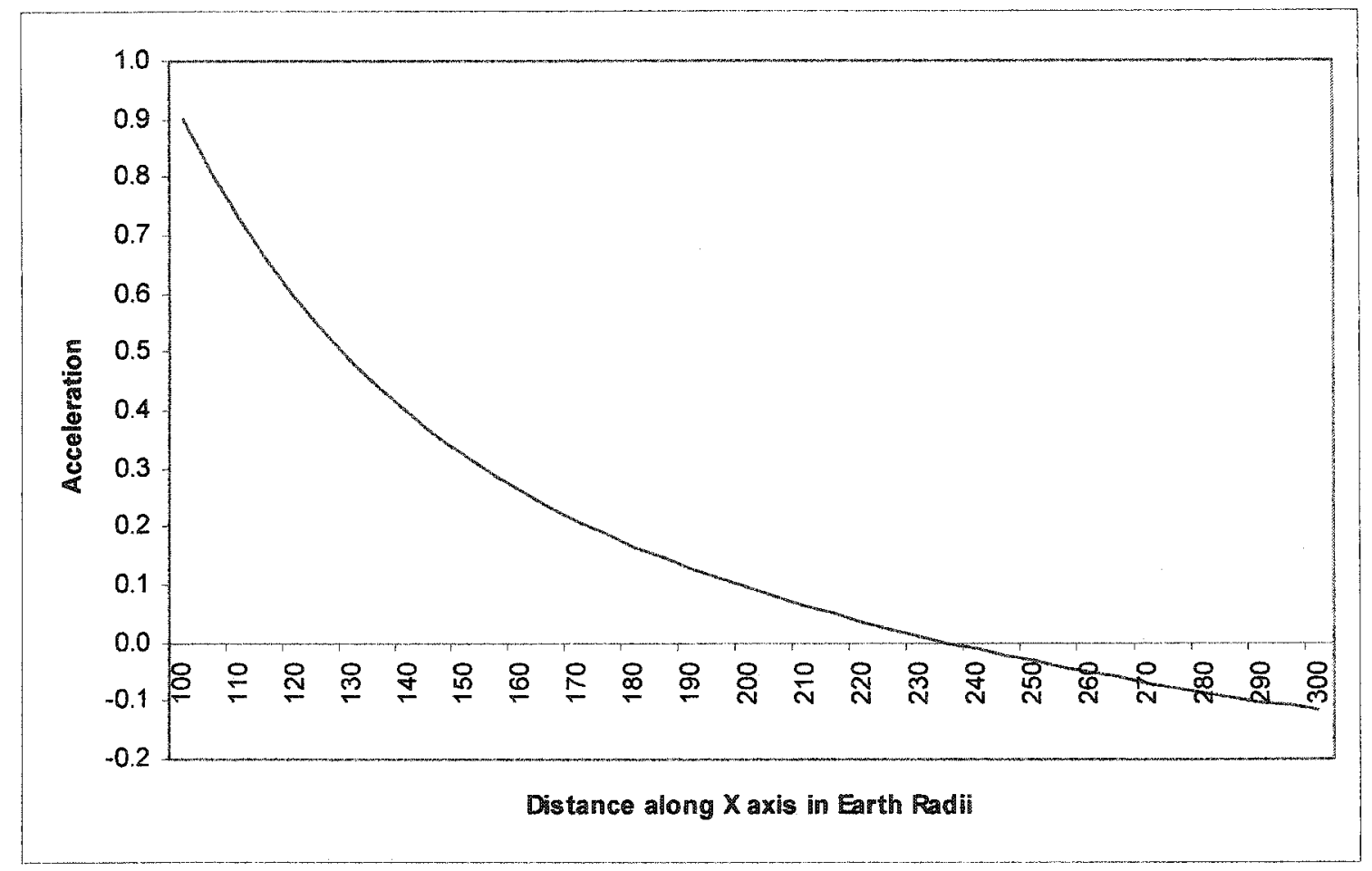

Figure 4.9 Characteristics of acceleration along the $\mathrm{x}$ axis required to maintain equilibrium in the SurEarth system. 
As expected, the acceleration required to maintain equilibrium will decrease as the solar sail gets closer to the $\mathrm{L}_{2}$ equilibrium point as depicted in figure 4.9. Once past the equilibrium point, a negative acceleration is required to counteract primarily the centrifugal acceleration. Since a solar sail can only derive an acceleration away from the Sun and not towards it, generating a negative acceleration is impossible and, therefore, the $L_{2}$ point becomes the maximum distance from which the solar sail may operate.

The next scenario to investigate is to keep the solar sail at $100 \mathrm{R}_{\mathrm{E}}$ along the $x$ axis and to manoeuvre out of plane along the $\mathrm{z}$ axis. Since there is no $y$ component, then (4.52) becomes:

$$
\vec{a}_{s s}=-\Omega^{2}\left(\begin{array}{l}
x \\
0
\end{array}\right)+\frac{G m_{1}}{\left[(x+\mu)^{2}+z^{2}\right]^{3 / 2}}\left(\begin{array}{c}
x+\mu \\
z
\end{array}\right)+\frac{G m_{2}}{\left[(x-(1-\mu))^{2}+z^{2}\right]^{3 / 2}}\left(\begin{array}{c}
x-(1-\mu) \\
z
\end{array}\right),
$$

where the component in the $\hat{\mathrm{i}}$ direction is:

$$
a_{s s_{x}}=\left[-\Omega^{2} x+\frac{G m_{1}(x+\mu)}{\left[(x+\mu)^{2}+z^{2}\right]^{3 / 2}}+\frac{G m_{2}(x-(1-\mu))}{\left[(x-(1-\mu))^{2}+z^{2}\right]^{3 / 2}}\right] \hat{\mathrm{i}} .
$$

If the desired $z$ distance of $27 R_{E}$ is substituted in (4.55), then:

$$
a_{s s_{x}}=0.80577 \mathrm{~mm} / \mathrm{s}^{2},
$$

and the component in the $\hat{\mathrm{k}}$ direction is:

$$
\begin{gathered}
a_{s s_{z}}=\left[\frac{G m_{1} z}{\left[(x+\mu)^{2}+z^{2}\right]^{3 / 2}}+\frac{G m_{2} z}{\left[(x-(1-\mu))^{2}+z^{2}\right]^{3 / 2}}\right] \hat{\mathrm{k}}, \\
a_{s s_{z}}=0.24478 \mathrm{~mm} / \mathrm{s}^{2} .
\end{gathered}
$$

Therefore the total acceleration at the position where $x=100 \mathrm{R}_{\mathrm{E}}$ and $z=27 \mathrm{R}_{\mathrm{E}}$ is:

$$
a_{s s}=\sqrt{{a_{s s_{x}}{ }^{2}+a_{s s_{z}}{ }^{2}}_{2}}=0.84213 \mathrm{~mm} / \mathrm{s}^{2} .
$$


The solar sail acceleration characteristics are depicted in the figure below.

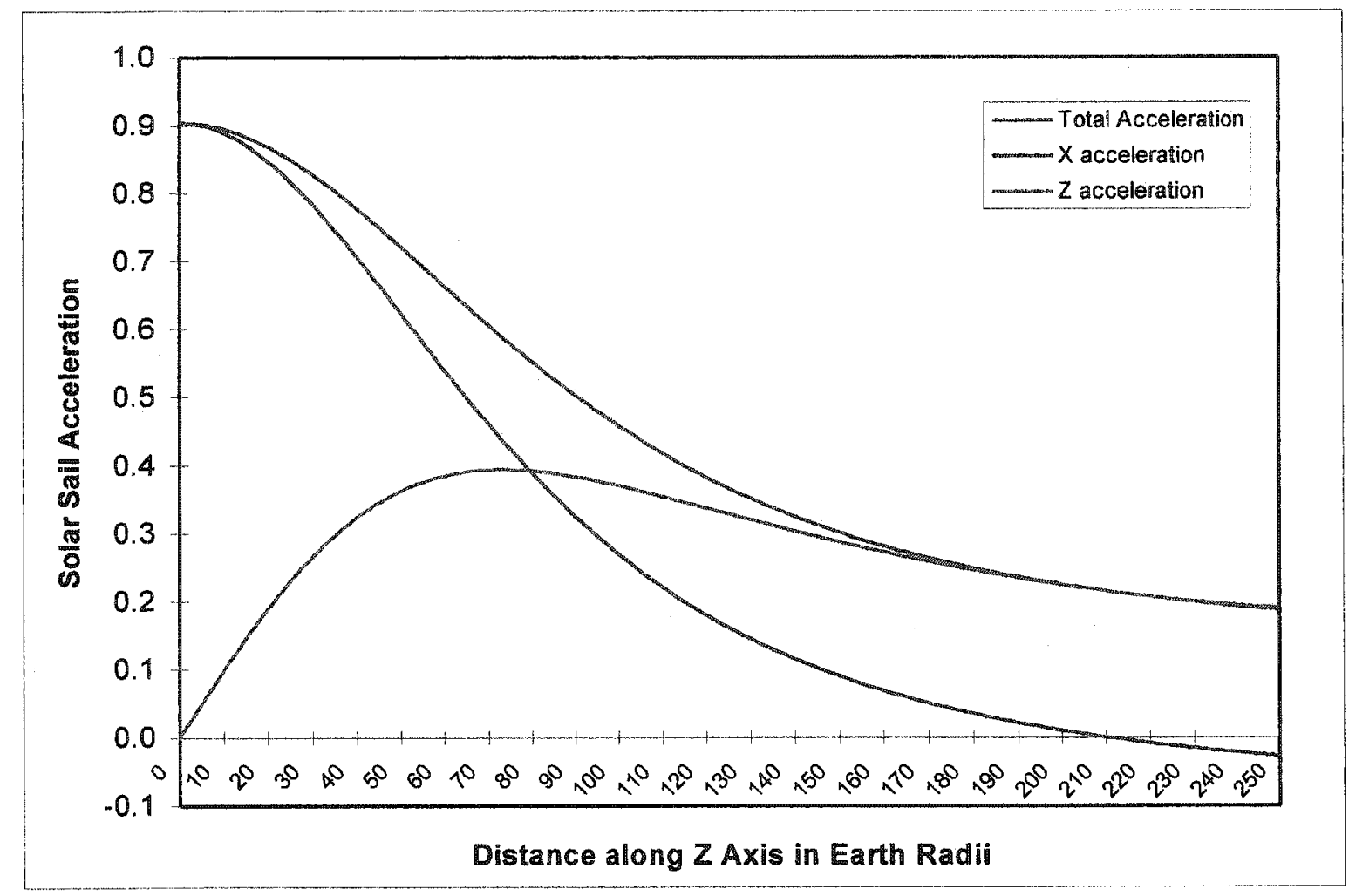

Figure 4.10 Solar sail acceleration characteristics at $100 \mathrm{R}_{\mathrm{E}}$ along the $x$ axis and increasing along the $z$ axis from 0 to $250 \mathrm{R}_{\mathrm{E}}$.

If the same process were repeated for analyzing the acceleration of the solar sail as it manoeuvres along the $y$ axis, the results would be fairly similar to figure 4.10 with the exception that there is a component of angular acceleration included along the $y$ axis which was not present when the solar sail manoeuvred along the $z$ axis. This can be seen in (4.52) which takes the form:

$$
\vec{a}_{s s}=-\Omega^{2}\left(\begin{array}{l}
x \\
y
\end{array}\right)+\frac{G m_{1}}{\left[(x+\mu)^{2}+y^{2}\right]^{3 / 2}}\left(\begin{array}{c}
x+\mu \\
y
\end{array}\right)+\frac{G m_{2}}{\left[(x-(1-\mu))^{2}+y^{2}\right]^{3 / 2}}\left(\begin{array}{c}
x-(1-\mu) \\
y
\end{array}\right)
$$

The angular acceleration component in the $\hat{j}$ direction has the effect of further reducing the required solar sail acceleration as the spacecraft manoeuvres along the $y$ 
axis. A comparison of acceleration characteristics for both the $z$ axis and $y$ axis is shown in figure 4.11 below.

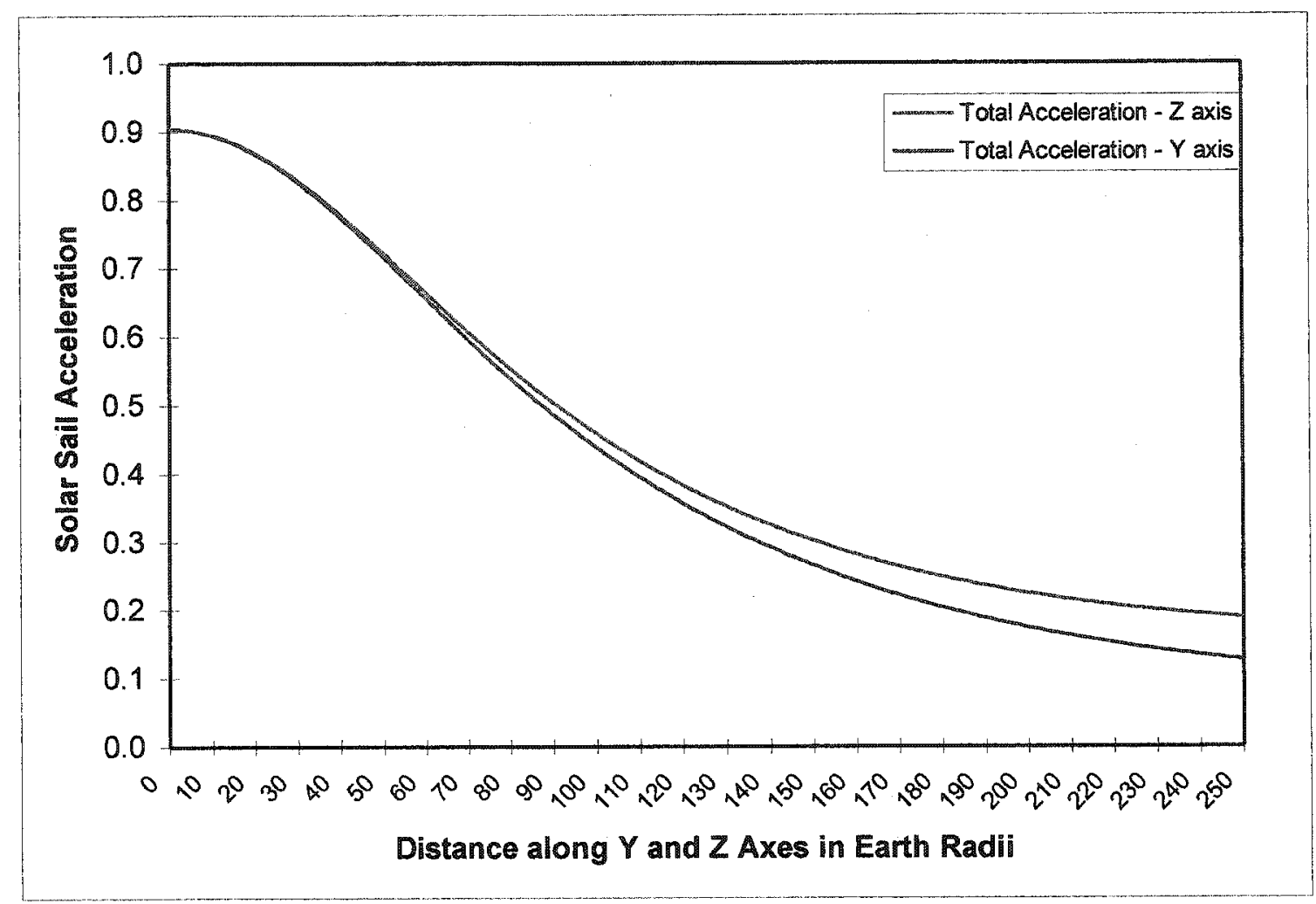

Figure 4.11 Solar sail accelerations along the $y$ and $z$ axes with the distance along the $x$ axis fixed at $100 R_{E}$.

The reduction in required solar sail acceleration as the spacecraft moves further along the $y$ and $\mathrm{z}$ axes can be explained by examining (4.52). From a balance of forces perspective, the solar sail is affected by three forces in the restricted three-body problem: the centrifugal force propelling it away from the Sun, and the gravitational forces of the Sun and Earth attracting it towards them. When considering the individual components of (4.52), both the angular acceleration and the Sun's gravitational acceleration are dominated mathematically by the large distance from the Sun to the Earth. Therefore, it appears on the surface that the attractive force of the Earth plays a minor role. However, the two primary forces (angular acceleration and the Sun's gravitational acceleration) 
tend to balance each other making the attractive force of the Earth the key component when determining the required solar sail acceleration. Therefore, the point of closest approach to the Earth would translate to the largest value of required acceleration. This point occurs at $100 \mathrm{RE}$ along the $x$ axis with the $y$ and $z$ components equal to zero as depicted in figures 4.10 and 4.11

The solar sail acceleration required to maintain equilibrium at $100 R_{E}$ was calculated to be $a_{s s}=0.9039 \mathrm{~mm} / \mathrm{s}^{2}$ for an ideal sail. To determine the sail loading $(\sigma)$, a form of (3.24) is used:

$$
\sigma=\frac{2 W_{E}}{a_{s s} c}\left(\frac{r_{E}}{r}\right)^{2},
$$

where $r$ represents the distance from the Sun to the solar sail at $100 \mathrm{R}_{\mathrm{E}}$ and $r_{E}$ represents the distance from the Sun to the Earth:

$$
\sigma=10.004 \mathrm{~g} / \mathrm{m}^{2}
$$

Substituting $\sigma$ into (3.23) and (3.26) will yield the characteristic acceleration and the sail lightness number respectively:

$$
\begin{gathered}
a_{0}=\frac{9.12}{\sigma}=0.9116 \mathrm{~mm} / \mathrm{s}^{2}, \\
\beta=\frac{1.529}{\sigma}=0.15284
\end{gathered}
$$

So far the performance metrics have been determined for an ideal solar sail with a reflectivity of $100 \%$. However, even highly reflective materials cannot reflect all light and there must be an efficiency attributed to the sail material. As previously mentioned in chapter 3 , Aluminum has a fairly high efficiency of 0.88 to 0.90 , making it the desired 
material for a solar sail. Given this fact, an efficiency of $\eta=0.88$ will be used to calculate the performance metrics for a real sail. Therefore, using the above metrics:

$$
\begin{gathered}
\sigma_{r}=\eta \times \sigma=8.8035 \mathrm{~g} / \mathrm{m}^{2} \\
a_{s s_{r}}=\frac{a_{s s}}{\eta}=1.02716 \mathrm{~mm} / \mathrm{s}^{2} \\
\beta_{r}=\frac{\beta}{\eta}=0.1737
\end{gathered}
$$

\subsection{Summary}

Throughout this chapter, solar sailing in a restricted three-body system was developed to analyze its viability for the proposed mission application of exploring the Earth's geomagnetic tail. The Lagrange equilibrium points were explored and then adapted to solar sailing showing the existence of a multitude of equilibrium contours depending upon the sail performance. The equilibrium contours would allow a solar sail propelled spacecraft to position itself out of plane and maintain its position as well as travel to other equilibrium positions within the envelope of its performance metrics.

The stability of the equilibrium contours was discussed and it was determined that they are fundamentally unstable, however, using a simple feedback control scheme would make the spacecraft completely controllable. The effects of the sail materials were also discussed showing that a reflectivity efficiency needs to be built in to account for imperfections in the reflective surface.

Finally, a concept for the mission application was developed from a force balance model to determine the acceleration that the solar sail would need to develop so that the desired distances from table 2.1 could be met. The resulting performance metrics are 
summarized in the following table. As expected, the performance of the solar sail must increase to maintain the desired distances of the mission application.

\begin{tabular}{|c|c|c|c|}
\hline & Acceleration & Sail Loading $\sigma$ & Lightness Number $\beta$ \\
\hline Ideal Sail & 0.9116 & 10.004 & 0.1528 \\
Real Sail & 1.02716 & 8.8035 & 0.1737 \\
\hline
\end{tabular}

Table 4.2 Solar sail performance metrics for an ideal and real sail for exploring the Earth's geomagnetic tail.

The forthcoming chapter will use the performance metrics provided in table 4.2 to develop further design parameters of the spacecraft including sail configuration, sail materials, spacecraft mass budget, as well as launching the spacecraft to the Earth's geomagnetic tail and manoeuvring within it. 


\section{Chapter 5 - Spacecraft Design}

With the development of the orbital dynamics for a solar sail in a restricted threebody system complete, further definition and analysis of the spacecraft design can now be provided. This chapter will cover the spacecraft structure including: potential solar sail configurations, sail materials, payload definition, spacecraft bus considerations and the Scisat-1 spacecraft as a possible platform on which to mount solar sails. As well, the Scisat-1 mass budget will be modified to allow for solar sail propulsion. It is important to note that Scisat-1 is a Low Earth Orbit (LEO) spacecraft and, therefore, extensive modifications would be required to actually adapt it to the geomagnetic tail environment.

From the development of a proposed mass budget, it will be possible to look at a launch vehicle for the spacecraft and discuss how the solar sails will propel the spacecraft from a circular Earth orbit to the equilibrium contours near the $\mathrm{L}_{2}$ point. Finally, the issue of controlling and steering the spacecraft within the Earth's geomagnetic tail will be investigated to determine a strategy for conducting the proposed mission application.

\subsection{Sail Configuration}

Throughout the history of solar sail development, a variety of sail configurations have been explored for various missions within the solar system and beyond. Each configuration has both pros and cons related to its design, depending upon the sail performance, deployment strategy and mission application. An exploration of each type of sail design will be carried out to determine the suitability for the mission application to the Earth's geomagnetic tail. Solar sails can be grouped under two main design 
categories: three-axis stabilized designs and spin stabilized designs. The three-axis stabilized designs require a support structure by which the solar sail material is kept in tension, while the spin stabilized designs use the centrifugal force of a spinning spacecraft to keep the sail material taut.

Several sail shapes have been proposed for a three-axis stabilized type of sail design but perhaps the most studied configuration is the square sail. JPL based the Halley comet rendezvous mission originally on this type of design but later decided to switch to the heliogyro due to the high risk associated with the sail deployment of the square sail. The following diagram shows an example of a three-axis stabilized square solar sail design.
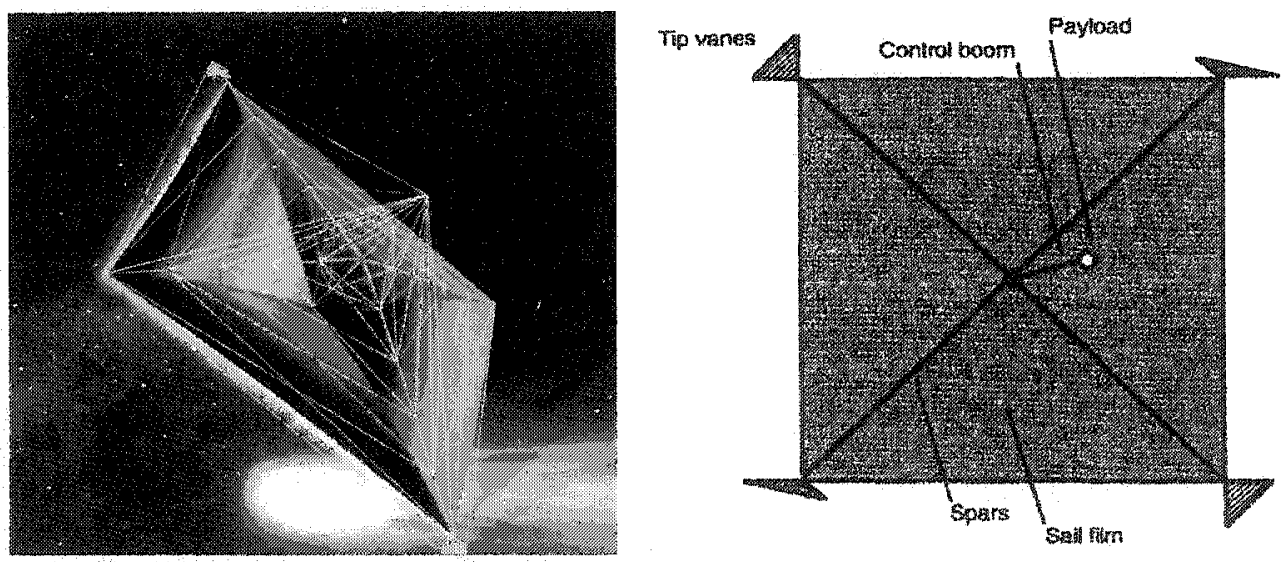

Figure 5.1 Artist's concept and schematic of a three-axis stabilized square solar sail design. (Courtesy NASA/JPL)

This sail design's main disadvantage resulted from the deployment of the sail structure and sail material. The sail spars would be deployed first followed by any supporting stays and then finally by the sail material itself as depicted in Figure 5.2.

Since each step in the deployment process relied on the success of the preceding step, any failure in the process would result in the sail being unable to be deployed. 

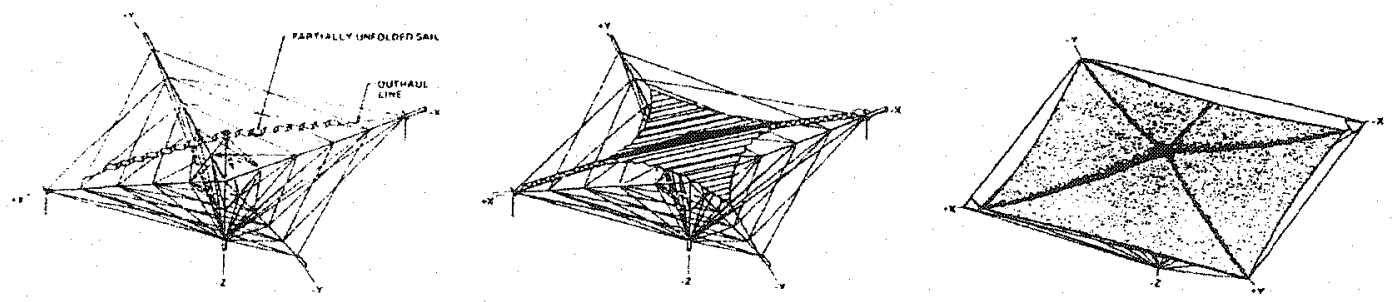

Figure 5.2 Deployment of a three-axis stabilized square solar sail. If any step in the deployment failed, the entire sail would be useless. (Courtesy NASA/JPL)

Also, redundancy in the deployment system is practically impossible due to the added weight and the nature of the design. This makes the risk of failure for the sail deployment quite high.

One advantage of this design, which is unlike a spinning solar sail, is the simplicity of the control mechanics. Control torques may be generated from a variety of methods including displacing the center of mass of the solar sail relative to the center of pressure or using small reflective vanes at the spar tips. Displacing the center of mass may be achieved by offsetting the payload on an articulated boom which can then be rotated, or by displacing the entire sail relative to the center of mass. The reflective vanes may be rotated to induce a torque and turn the sail to the desired attitude. ${ }^{105}$

One other aspect of sail design which must be investigated relates to the proposed mission application of this thesis. Since the objective of the mission is to explore the geomagnetic tail, the solar sail must have the ability to not only park itself to maintain a specific position, but also manoeuvre in any direction to a new position. Therefore, the solar sail must have the ability to vary the acceleration generated so that a variety of positions may be maintained given the fact that the required acceleration reduces as the

${ }^{105}$ Colin R. McInnes, Solar Sailing: Technology, Dynamics and Mission Applications, Praxis Publishing Ltd., Chichester, UK, 1999, pp. 76-81. 
spacecraft gets closer to the $L_{2}$ point. Since acceleration generated by the solar sail is directly related to sail area, the solar sail design must be able to vary the sail area in order to control the acceleration. The square sail configuration is unable to vary sail area and, therefore, proves to be an unsuitable design for the proposed mission application.

The other category of solar sail design is the spin stabilized type. MacNeal invented this design in the 1960's and called it the heliogyro. One of the main advantages for a spinning solar sail is that there is no additional structure required to keep the sail material taut since the blades are kept in tension by centrifugal force. The other
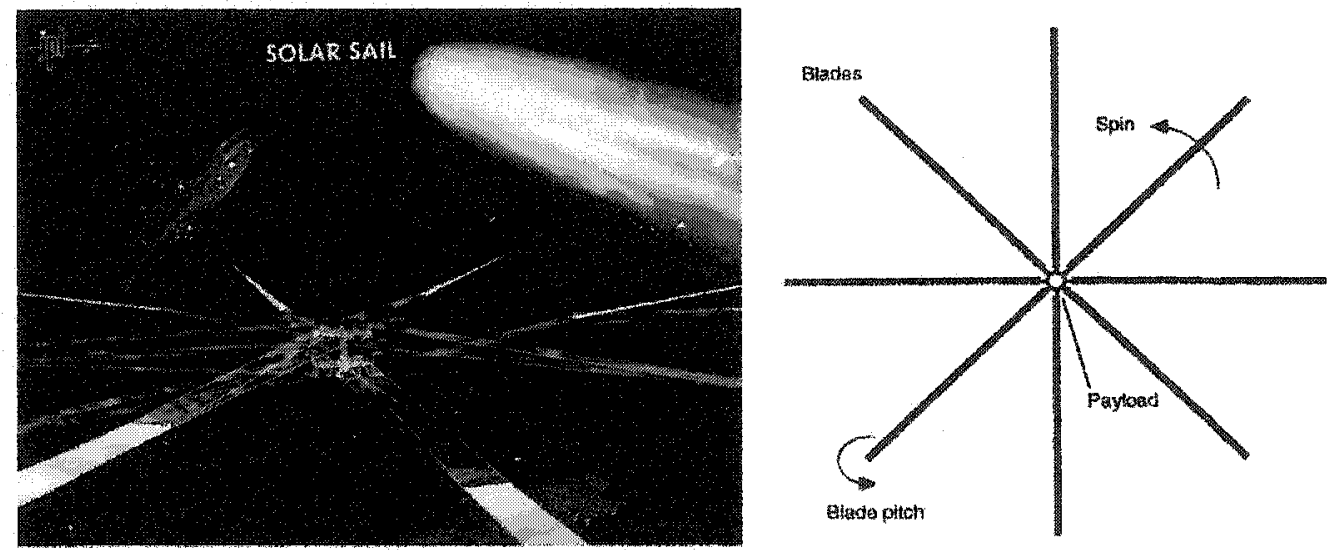

Figure 5.3 Artist's concept and schematic of a heliogyro. Spacecraft orientation is controlled by pitching the blades at intervals. (Courtesy NASA/JPL)

advantage is that the deployment mechanism is very simple since the heliogyro "blades" are kept on reels. After the spacecraft has been launched, the central control unit is spun up to a specific angular velocity. Then opposing blades are deployed slowly until their full length is reached resulting in a much lower risk of failure. While the reduction in structural mass appears to reduce the overall mass of the spacecraft, "it has been found that for a large heliogyro the additional mass required to provide blade torsional stiffness may in fact reduce some of the apparent advantages of the concept."106

${ }^{106}$ Ibid. p. 83. 
Perhaps the greatest disadvantage of this design is the very complicated control mechanism. The heliogyro is controlled by varying the blade pitch, much like a helicopter, in a "collective and cyclic manner". 107 The "collective manner" means that the blades are all tumed the same amount resulting in a change to the spin rate of the spacecraft. The "cyclic manner" means that the blades are turned during a certain portion of the rotation, which will generate a torque to change the orientation of the spacecraft. The combination of these two control manoeuvres will steer the spacecraft to the desired orientation, however, this control strategy is very complicated.

If the heliogyro is applied to the mission application requirements of this thesis, the design may be able to vary the acceleration produced by the solar sail blades; however, this will complicate the control strategy even more. The only way to vary the acceleration would be to either shorten the blade length by reeling in some material or to orient the blades to reduce the amount of area exposed to sunlight. Unfortunately, reeling in blade material will cause the spacecraft to spin faster, which will create added complications to any steering algorithms. Also, varying the blade angle to reduce sail area exposed to the Sun could change spacecraft angular velocity. unless the blades are turned in such a way as to negate the additional transverse forces. If these scenarios were coupled with the already complicated control scheme, then the risk of making a mistake, and sending the spacecraft out of control increases exponentially. For this reason, a spinning solar sail configuration should not be considered unless absolutely necessary.

While there are hybrid designs which incorporate aspects of either a spinning solar sail or three-axis stabilized type, not all of them meet the desired requirements of

${ }^{107}$ lbid. p. 88. 
the geomagnetic tail mission. One design proposed by Bernasconi and Zurbuchen may be able to fulfill the design requirements and also provide a fairly safe deployment strategy. In their article entitled "Lobed Solar Sails for a Small Mission to the Asteroids," they describe a new solar sail configuration using rigidized inflatable technology.

The shape of the solar sail looks like a four-leaf clover where each leaf or "saillet" can be operated independently of the others. As described in Bernasconi and Zurbuchen's article, the saillets are round in shape, with a rigidizing material along the circumference, forming a torus. Upon deployment, the rigidizable laminate is inflated with Nitrogen gas to form the torus and flatten the sail material. Over a period of time, the laminate is cured so that the torus becomes a rigid material to support the solar radiation pressure force applied to the saillet. With some minor modifications to the sail shape, the spacecraft used for the geomagnetic tail mission could look like Figure 5.4.

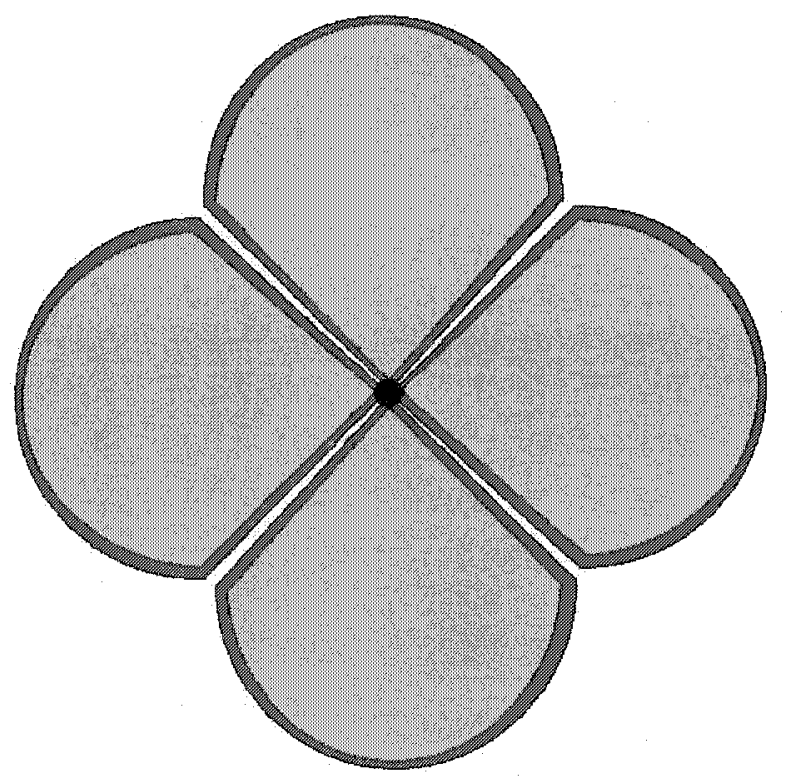

Figure 5.4 Cloverleaf sail geometry. Each saillet can be tilted independently. 
There are numerous advantages associated with this sail configuration. Perhaps the most important for the proposed mission to the geomagnetic tail is that the solar radiation pressure acceleration can be controlled by simply tilting each saillet to reduce the surface area exposed to the Sun. As long as the saillets are tilted so as to negate any adverse transverse forces, the spacecraft will maintain its position. This strategy will allow the spacecraft to position itself anywhere within the operational envelope of the geomagnetic tail from $100 \mathrm{R}_{\mathrm{E}}$ to the $\mathrm{L}_{2}$ equilibrium point.

Another major advantage of this configuration is that it is a three-axis stabilized design, which means the control of the solar sail is fairly straightforward, unlike a spinning design. Also, due to the large surface area of each saillet, high manoeuvring control torques can be generated to change the solar sail attitude quickly. As well, since there is no requirement for additional support structures such as spars or booms, the overall mass of the spacecraft is reduced.

Finally, the risk of failure during deployment is greatly reduced since there is no additional support structure. Each saillet is simply released from a storage housing and the rigidizable laminate torus is inflated. Once cured, the solar sail is ready to operate. As well, there is redundancy in the system, since a failure of one saillet would not jeopardize the entire mission. The spacecraft could still operate with the remaining three saillets, however, the sail performance would be degraded and the mission would have to be modified to operate closer to the $\mathrm{L}_{2}$ point.

The cloverleaf configuration of solar sail appears to combine the benefits of the three-axis stabilized square sail and the heliogyro design. It has a lower risk deployment 
scheme like the heliogyro and the simplified control strategy of the square sail. In addition, it can also vary the solar radiation pressure acceleration generated by the saillets so that manoeuvring within the geomagnetic tail is possible. Therefore, the cloverleaf solar sail configuration will be the optimum design for the proposed mission application being explored by this thesis.

\subsection{Sail Materials}

A very important design consideration for a solar sail is the choice of material which will be used to reflect the sunlight. The science of the structure of materials and their manufacture has advanced significantly over the past century, especially with the requirement for strong, lightweight composites for the space industry. For a solar sail, the requirement for a lightweight material is even more imperative since a major performance metric of a solar sail propelled spacecraft is the sail loading $\left(\mathrm{g} / \mathrm{m}^{2}\right)$. Since the sail loading is directly proportional to the overall spacecraft mass, the lighter the material per unit area, the better the solar sail performance.

The structure of sail material can be broken down into two main components: the sail film or substrate and the reflective coating. The substrate is the portion of the sail material to which the reflective coating is adhered. As well, the substrate "is required to allow handling, folding, packing and deployment of the sail film." 108 This material must also possess sufficient tensile strength to mitigate any tearing once the solar radiation pressure is applied. The reflective coating is needed to reflect the sunlight efficiently so that the performance of the solar sail may be maximized. If the reflective coating allows too much transmittance, then the sail performance will be degraded. 
There are a number of substrate materials available from the commercial market including Kapton, Mylar and Lexan. ${ }^{109}$ The characteristics of these materials are captured in the following table.

\begin{tabular}{|c|c|c|c|c|c|c|}
\hline & $\begin{array}{c}\text { Bulk } \\
\text { Density } \\
\left(\mathrm{g} / \mathrm{cm}^{3}\right)\end{array}$ & $\begin{array}{c}\text { Tensile } \\
\text { Strength } \\
\left(\mathrm{N} / \mathrm{m}^{2}\right)\end{array}$ & $\begin{array}{c}\text { Tensile } \\
\text { Modulus } \\
\left(\mathrm{N} / \mathrm{m}^{2}\right)\end{array}$ & \multicolumn{3}{|c|}{ Surface Density $\left(\mathrm{g} / \mathrm{m}^{2}\right)$} \\
\cline { 5 - 7 } Kapton & 1.42 & $1.72 \times 10^{8}$ & $2.96 \times 10^{9}$ & 1.42 & 4.26 & $5 \mu \mathrm{m}$ \\
Mylar & 1.38 & $1.72 \times 10^{8}$ & $3.79 \times 10^{9}$ & 1.38 & 4.14 & 6.90 \\
Lexan & 1.20 & $6.89 \times 10^{7}$ & $2.07 \times 10^{9}$ & 1.20 & 3.60 & 6.00 \\
\hline
\end{tabular}

Table 5.1 Properties of substrate materials. (Source McInnes, 1999.)

While Lexan would appear to be a preferred material due to its surface density, it actually reacts very poorly to UV rays. The preferred material is Kapton due to the fact that it is "essentially chemically inert, has a high resistance to radiation and maintains its physical and mechanical properties over a wide range of temperatures." ${ }^{9110}$ Kapton also has very good adhesion qualities for attaching the reflective coating. McInnes has stated that Kapton is currently available at $7.6 \mu \mathrm{m}$, but that experiments have demonstrated the ability to produce a film with a thickness of $0.9 \mu \mathrm{m}$. Also, for a moderate performance solar sail, he recommends a substrate thickness of $2 \mu \mathrm{m} .{ }^{111}$ Given that the performance of the solar sail for the geomagnetic tail mission can be considered moderate, it is assumed that a substrate thickness of $2 \mu \mathrm{m}$ could be manufactured which will have a surface density of $2.84 \mathrm{~g} / \mathrm{m}^{2}$.

\footnotetext{
${ }^{108}$ Ibid. p. 60.

${ }^{109}$ Kapton $(B)$ Mylar $(\mathbb{B}$ and Lexan $(\mathbb{B})$ are all registered trademarks of duPont Ltd.

${ }^{110}$ Colin R. McInnes, Solar Sailing: Technology, Dynamics and Mission Applications, Praxis Publishing Ltd., Chichester, UK, 1999, pp. 61-62.

${ }^{111}$ Ibid, p.62.
} 
There are several materials which could be used for a reflective coating, however, aluminum "has the best overall reflectance across the spectrum."112 The physical properties of several sail film reflective coatings are listed in the following table.

\begin{tabular}{|c|c|c|c|c|c|}
\hline & $\begin{array}{c}\text { Bulk Density } \\
\left(\mathrm{g} / \mathrm{cm}^{3}\right)\end{array}$ & \multicolumn{3}{|c|}{ Surface Density $\left(\mathrm{g} / \mathrm{m}^{2}\right)$} & \multirow{2}{*}{$\begin{array}{c}\text { Melting Point } \\
(\mathrm{K})\end{array}$} \\
\cline { 3 - 5 } & 2.70 & $0.1 \mu \mathrm{m}$ & $0.3 \mu \mathrm{m}$ & $0.5 \mu \mathrm{m}$ & 933 \\
Aluminum & 0.27 & 0.81 & 1.35 & 933 \\
Lithium & 0.53 & 0.05 & 0.16 & 0.27 & 453 \\
Silver & 10.5 & 1.05 & 3.15 & 5.25 & 1234 \\
\hline
\end{tabular}

Table 5.2 Properties of reflective coatings. (Source McInnes, 1999.)

The primary disadvantage for lithium is its low melting point, while the disadvantage for silver is that it has a higher bulk density than aluminum. Figure 5.5 illustrates a spectral reflectance problem also associated with silver.

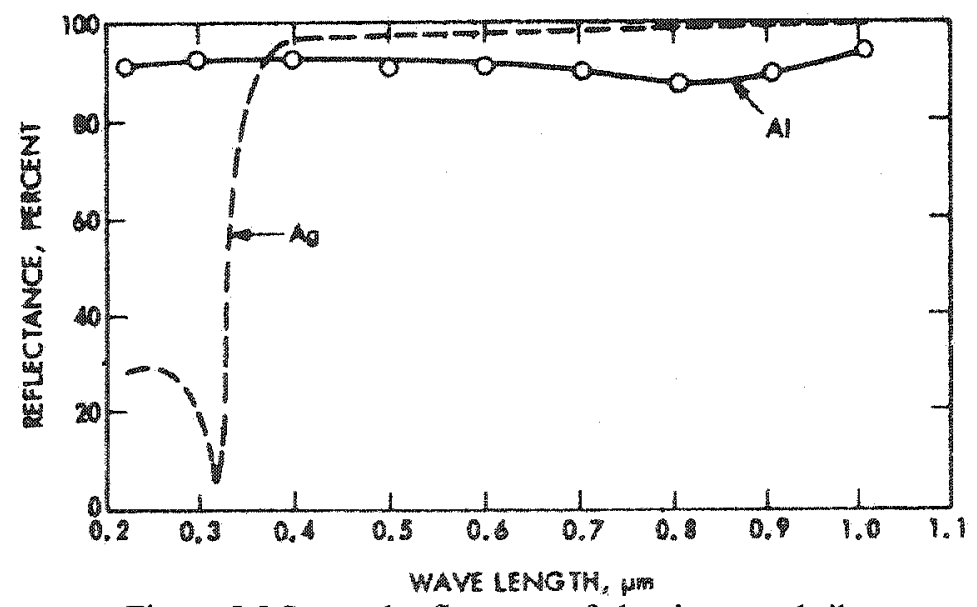

Figure 5.5 Spectral reflectance of aluminum and silver. (From Wright, 1992.)

Silver has a transparent window in the UV region which "would allow UV radiation to

112 Jerome L. Wright, Space Sailing, Gordon and Breach Science Publishers, The Netherlands, 1992, p. 101. 
penetrate and possibly degrade the underlying polymer film."113 Some other disadvantages of silver are its "high cost and its susceptibility to tarnishing in the prelaunch environment." 114 Aluminum does not have the reflectance problem as illustrated in Figure 5.5 so long as the material is applied with a minimum thickness of $1000 \mathrm{~A}$ or $0.1 \mu \mathrm{m}$. Therefore, for the proposed mission application to the geomagnetic tail, a value of $0.27 \mathrm{~g} / \mathrm{m}^{2}$ will be used for the surface density of the aluminum reflective coating.

Now that the substrate film and the reflective coating have been chosen, additional types of coatings must be considered to help protect the sail material. For a mission involving a close solar approach, a high-emissivity back coating may be required to perform "passive thermal control of the sail film by re-radiation." 115 By using the following equation it is possible to determine the equilibrium temperature of the sail:

$$
T=\left[\frac{1-\tilde{r}}{\varepsilon_{f}-\varepsilon_{b}} \frac{W_{E}}{\widetilde{\sigma}}\left(\frac{r_{E}}{r}\right)^{2} \cos \alpha\right]^{1 / 4},
$$

where $\tilde{r}$ is the reflectivity efficiency, $\widetilde{\sigma}$ is the Stefan-Boltzmann constant, $\varepsilon_{f}$ is the emissivity of the front surface and $\varepsilon_{b}$ is the emissivity of the back surface. By assuming a value for $\widetilde{r}$ of $0.85, \varepsilon_{f}=0$ and the fact that uncoated aluminized Kapton has an $\varepsilon_{b}$ value of 0.34 , it is possible to determine the equilibrium temperature of the sail for various distances and sail angles as depicted in Figure 5.6.

\footnotetext{
${ }^{113}$ W.M. Rowe, E.E. Luedke, and D.K. Edwards, "Thermal Radiative Properties of Solar Sail Film Materials", $2^{\text {nd }}$ AIAA/ASME Thermophysics and Heat Transfer Conference, Palo Alto, California, May 24 26, 1978, p. 2.

${ }^{114} \mathrm{lbid}$.

${ }^{115}$ McInnes, p. 63.
} 


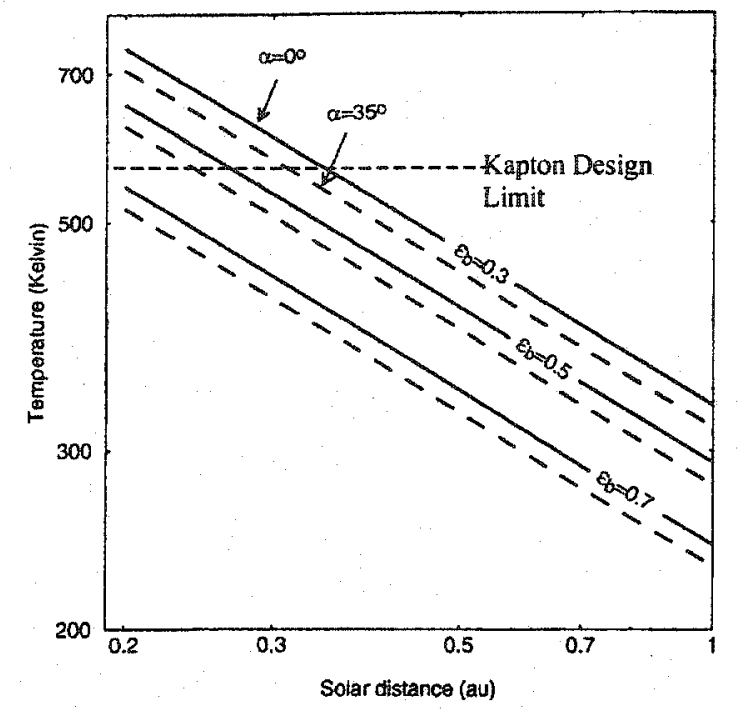

Figure 5.6 Plots of equilibrium temperature vs. solar distance. (From Mclnnes, 1999.)

Since the geomagnetic tail mission is going to operate beyond $1 \mathrm{AU}$, the equilibrium temperature will be quite low. In fact, since the emissivity of aluminized Kapton is already 0.34 , there is no requirement for any additional back coatings for passive thermal control. Therefore the cross section of the sail material will be as depicted in figure 5.7 below.

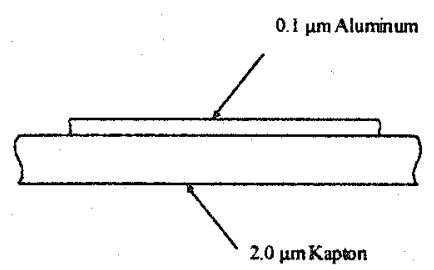

Figure 5.7 Cross section of sail material for geomagnetic tail mission.

A final consideration for the construction of the sail material is the mass of seams, ripstops and rigidizable laminate. Since the material is manufactured in strips rather than one large sheet, a seam is required to join the pieces together. As well, to prevent the propagation of accidental rips or tears, ripstops can be used in conjunction with seams to keep any tears localized. The additional material required for seams and ripstops will add 
a small amount to the mass properties of the sail. Wright has recommended that an allowance of $0.06 \mathrm{~g} / \mathrm{m}^{2}$ be added for this additional material. Also, since the sail material must be conductive to prevent the build up of electric charges in localized "hot spots", each seam and ripstop will have to be bonded with a grounding strap. ${ }^{116}$ A sample cross section of a solar sail panel seam is illustrated in figure 5.8.

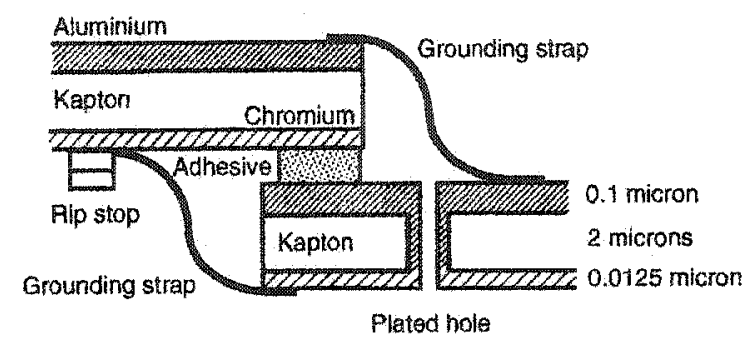

Figure 5.8 Cross section of bonded solar sail film panels. Note that chromium is used as a back emissivity film which is not required for the geomagnetic tail mission. (From McInnes, 1999.)

For the rigidizable laminate material, the article by Bernasconi and Zurbuchen gives the following table of physical properties:

\begin{tabular}{|c|c|c|}
\hline & $\begin{array}{c}\text { Basic Single-Ply } \\
\text { Laminate }\end{array}$ & $\begin{array}{c}\text { Reinforced Laminate } \\
\text { (Two-Ply) }\end{array}$ \\
\hline Membrane Stiffness & $0.5 \mathrm{MN} / \mathrm{m}$ & $0.8 \mathrm{MN} / \mathrm{m}$ \\
Surface Density $\left(\mathrm{g} / \mathrm{m}^{2}\right)$ & 108 & 185 \\
Thickness & $0.145 \mathrm{~mm}$ & $0.310 \mathrm{~mm}$ \\
\hline
\end{tabular}

Table 5.3 Properties of rigidizable laminate material. (Source Bernasconi \& Zurbuchen, 1995.)

The surface density of this material seems to be extremely high, however, its important to keep in mind that the total amount of area of the torus will be quite small as compared to the sail material itself. For the proposed sail configuration, there is probably no requirement for a reinforced laminate so that the basic single-ply material will be used for all mass calculations. By referring to Bernasconi and Zurbuchen's work, it is possible to

${ }^{116}$ Wright, p. 118. 
infer an area ratio for the rigidizable laminate as compared to the total sail area. The rigidizable laminate is calculated to be approximately $3.2 \%$ of the total area so that the sail material occupies the remaining $96.8 \%$. By applying the area percentages to the surface densities and then adding them together, a total sail material surface density can be calculated so that the rigidizable laminate is included.

In order to calculate the total mass of sail material required for the proposed solar sail mission, the following materials will be used as an optimum configuration for the sail.

\begin{tabular}{|c|c|c|c|}
\hline Item & Type & Thickness & Surface Density \\
\hline Substrate & Kapton® & $2.0 \mu \mathrm{m}$ & $2.84 \mathrm{~g} / \mathrm{m}^{2}$ \\
Reflective Coating & Aluminum & $0.1 \mu \mathrm{m}$ & $0.27 \mathrm{~g} / \mathrm{m}^{2}$ \\
Emitter & Nil & 0 & 0 \\
Seams and Ripstops & Kapton/Al & & $0.06 \mathrm{~g} / \mathrm{m}^{2}$ \\
Total Sail Material & & & $3.17 \mathrm{~g} / \mathrm{m}^{2}$ \\
$96.8 \%$ Sail & & & $3.068 \mathrm{~g} / \mathrm{m}^{2}$ \\
$3.2 \%$ Laminate & & & $3.456 \mathrm{~g} / \mathrm{m}^{2}$ \\
Total & & & $6.524 \mathrm{~g} / \mathrm{m}^{2}$ \\
\hline
\end{tabular}

Table 5.4 Summary of properties for solar sail material design.

\subsection{Payload Definition}

Since the objective of the proposed mission is to observe the plasma and magnetic field interactions within the geomagnetic tail, an appropriate scientific payload must be defined. Although an ideal scenario would allow for a rather comprehensive scientific instrument suite, the main concem for this mission is payload mass. Therefore, the 
instruments chosen must be not only capable of analyzing the interactions inside the geomagnetic tail, but must also be as minimal a mass as possible.

Fortunately the development of space instrument technology has progressed significantly in the recent past to allow for very "light weight" instruments. Bernasconi and Zurbuchen have suggested that three instruments would be required to study the composition of the interstellar space: an interplanetary gas instrument, a plasma particle detector and a magnetometer with a total mass of $17 \mathrm{~kg} .{ }^{117}$ During a recent symposium held by the Royal Astronomical Society on May $10^{\text {th }}, 2002$, a presentation was made entitled "GeoSail: Exploring Geospace Using a Small Solar Sail". 118 In the presentation, the author outlined the payload required to explore the geomagnetic tail which is detailed in the following table.

\begin{tabular}{|c|c|c|}
\hline Instrument & Mass (kg) & Science \\
\hline Flux-Gate Magnetometer & 0.23 & High resolution 3D magnetic field measurements \\
\hline Electrostatic Analyzer & 4.2 & $\begin{array}{l}\text { Measures 3D thermal/super-thermal electrons at energies } \\
50 \mathrm{eV}-10 \mathrm{keV} \text {. }\end{array}$ \\
\hline Solid State Telescope & 2.2 & $\begin{array}{l}\text { Measures super-thermal 3D ions \& electrons at higher } \\
\text { energies of } 10-100 \mathrm{keV}\end{array}$ \\
\hline Search-Coil Magnetometer & 1.7 & $\begin{array}{l}\text { Rapid variations of magnetic field in interaction region } \\
\text { between solar wind and magnetosphere. }\end{array}$ \\
\hline 3-axis Electric Field Instrument & 4.0 & Electric field measurements. \\
\hline DPU Processing & 1.1 & \\
\hline Total Mass & $13.4 \mathrm{~kg}$ & Total Power $\sim 7 W$ \\
\hline
\end{tabular}

Table 5.5 GeoSail Payload mass budget presented at Solar Sail Mission Applications Seminar. (From Macdonald, 2002.)

\footnotetext{
${ }^{117}$ Marco C. Bernasconi and Thomas Zurbuchen, "Lobed Solar Sails for a Small Mission to the Asteroids", Acta Astronautica Vol. 35, Supplement, 1995, p. 647.

${ }^{118}$ Malcolm Macdonald, "GeoSail: Exploring Geospace Using a Small Solar Sail", Solar Sail Mission Applications Seminar, London, England, 10 May 2002.
} 
Therefore, based on the work by the aforementioned authors, it is possible to define a payload for the geomagnetic tail mission which has a mass less than $20 \mathrm{~kg}$. However, to allow for possible expansion, a mass budget of $20 \mathrm{~kg}$ and a power budget of $10 \mathrm{~W}$ will be used for the solar sail mission.

\subsection{Spacecraft Bus Considerations}

The detailed design of a spacecraft bus for the proposed mission is beyond the scope of this thesis, however, several points must be considered should this task be undertaken.

The most important criteria for the spacecraft bus will be a necessarily minimum amount of mass. While this is always an important factor for conventional satellites, it is even more important for a solar sail because mass is directly proportional to sail area and, therefore, amount of sail material required for the mission objectives. For a solar sail mission, a spacecraft bus will need to accommodate the following components: Attitude Determination and Control System (ADCS), Command and Data Handling, Communications, Power, Thermal subsystem, Sail Deployment and Payload.

As mentioned previously, the sail configuration proposed for the geomagnetic tail mission is a cloverleaf design with a central spacecraft bus. The shape of the bus can be either a short cylinder or square since the saillets will be attached at four points equidistant around it. To control each saillet, a rotary actuator or stepper motor will be used to turn an attachment point for the sail material. The attachment point will need to incorporate a stabilizing plate attached to the spacecraft structure to prevent undue loading on the actuator from the solar sail. The attachment point must be designed so 
that the sail material and rigidizable laminate are held without creating undue stress in the materials which could lead to tears. As well, the attachment point will need a means of inflating the torus with Nitrogen during sail deployment and then maintaining a constant small pressure during sail operation.

Rotary actuators or stepper motors range in size depending upon design criteria. In their article, Bernasconi and Zurbuchen have given a mass estimate of the saillet actuators of $3.37 \mathrm{~kg}$ or $0.85 \mathrm{~kg}$ each. Also, a search of the Starsys Research Corporation website revealed a range of actuators from 0.335 to $4.365 \mathrm{~kg}$ each depending upon the application. Given the complexity of the stepper motor function coupled with a flexible system such as a solar sail, it would be impossible to pinpoint the exact actuator characteristics required to drive the system. Therefore, the Starsys HSR2320 rotary actuator will be used an example for the purpose of developing the mass budget. Each HSR 2320 has a mass of $1.1 \mathrm{~kg}$ and a step size of 0.0625 degrees. As well, the actuator has a maximum powered holding torque of $20.9 \mathrm{Nm}$ which should be sufficient to overcome the frictional forces from bearings. If an additional $0.4 \mathrm{~kg}$ is added for each solar sail attachment point, the total mass budget of the control system is $6 \mathrm{~kg}$.

Now that a method of controlling the solar sails has been suggested, a strategy for navigation can be explored. With the operating region being greater than $100 R_{E}$ away from the Earth, the spacecraft will be required to navigate autonomously by the ADCS. As this is a three-axis stabilized spacecraft, the ADCS will need to determine the spacecraft orientation as well as the position in space relative to the Sun and Earth. To accomplish this task, a number of navigation instruments are possible for use on the spacecraft bus including: Sun sensors, star sensors, horizon sensors and magnetometers. 
Since the geomagnetic tail mission will be oriented towards the Sun, a Sun sensor would be an obvious choice to provide one axis of orientation. An Earth sensor may be possible, however, the spacecraft will not always be pointed towards the Earth and, therefore, it may be outside of the instrument's field of view. Both the magnetometer and horizon sensor would not be useful given the distance away from the Earth. Finally, star sensors would be extremely beneficial for providing not only spacecraft orientation but also inertial position in space. "By using at least two star vectors separated by some angular distance, full three-axis knowledge is available." 119 Therefore, for the geomagnetic tail mission to navigate autonomously, the ADCS will require one Sun sensor and a minimum of one star sensor to determine spacecraft orientation and inertial position relative to the Sun and Earth.

Another component of the spacecraft bus specific to the proposed solar sail mission is the pressurization sub-system used during sail deployment. Bernasconi and Zurbuchen describe a pressurization system with a mass of $20.95 \mathrm{~kg}$ which consists of Nitrogen storage bottles, plumbing, valves and a control mechanism. The system maintains the pressure of the tori at $1.62 \mathrm{kPa}$ so that the rigidizable laminate material has time to cure over a six-hour period. ${ }^{120}$ They also derived the volume of 2.4 litres for each pressure vessel based on a storage pressure of $21 \mathrm{MPa}$. Once the sail area has been derived for the geomagnetic tail mission, the volume of Nitrogen can be calculated to size the storage tanks. Since the proper deployment of the solar sails is mission critical,

\footnotetext{
119 Wiley J. Larson and James R. Wertz, Space Mission Analysis and Design Second Edition, Microcosm Inc., USA and Kluwer Academic Publishers, The Netherlands, 1992, p. 361.

${ }^{120}$ Marco C. Bernasconi and Thomas Zurbuchen, "Lobed Solar Sails For A Small Mission to the Asteroids", Acta Astronautica, Vol. 35. Supplement, 1995, p. 651.
} 
redundancy must be designed into the pressurization system. This may be accomplished by designing the system to feed the solar sails with redundant channels or valves as backup. System redundancy is the reason why Bernasconi and Zurbuchen's pressurization system mass budget is approximately $21 \mathrm{~kg}$.

\subsection{The Magellan Aerospace Corporation Scisat-1 Platform}

In December 2002, a small scientific satellite will be launched from a Pegasus XL launch vehicle into a 650 kilometer orbit about the Earth. The satellite is called Scisat-1 and will be a joint Canadian Space Agency (CSA)/National Aeronautics and Space Administration (NASA) venture to measure the makeup of the Earth's atmosphere.

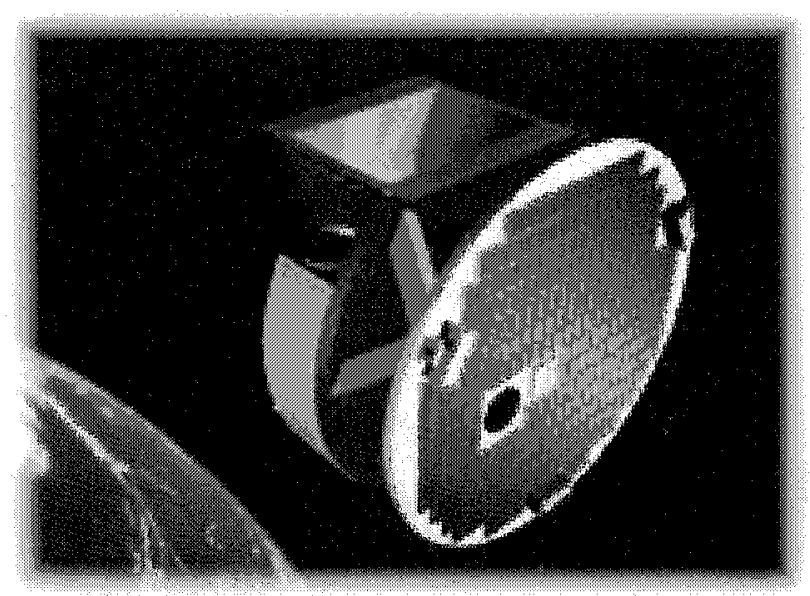

Figure 5.9 Artist's rendering of the Scisat-1 satellite. (Courtesy Magellan Aerospace Corporation, 2002.)

The Scisat-1 platform has been designed for launch on a Pegasus XL vehicle which means it is relatively compact in size and light weight. This may make it an ideal candidate for the spacecraft bus of a solar sail mission. Perhaps the two main advantages of considering this vehicle is cost effectiveness and reliability, since the Scisat-1 spacecraft will be completely researched and flown later this year. As well, by using an already integrated system, a great deal of research and development time can be saved by 
adapting it to solar sail propulsion. Therefore, as a proposal for the spacecraft design, the Scisat-1 satellite will be used to further develop the mission application to the geomagnetic tail. The Scisat-1 mass and power budget is broken down as follows:

\begin{tabular}{|c|c|c|}
\hline Component & Mass $(\mathrm{kg})$ & Power $(\mathrm{W})$ \\
\hline $\begin{array}{c}\text { Attitude Determination and } \\
\text { Control System (ADCS) }\end{array}$ & 21.26 & 15 \\
Command and Data Handling & 3.25 & 7 \\
Communications & 5.45 & 10.5 \\
Harness & 8.86 & 0 \\
Power & 12.52 & 9 \\
Structure & 52.73 & 0 \\
Thermal & 6.73 & 3 \\
Payload & 50 & 30 \\
Total & $\mathbf{1 6 0 . 8}$ & $\mathbf{7 4 . 5 W}$ \\
\hline
\end{tabular}

Table 5.6 Mass and power budgets for Scisat- 1 spacecraft. (Source Magellan Aerospace, 2002.)

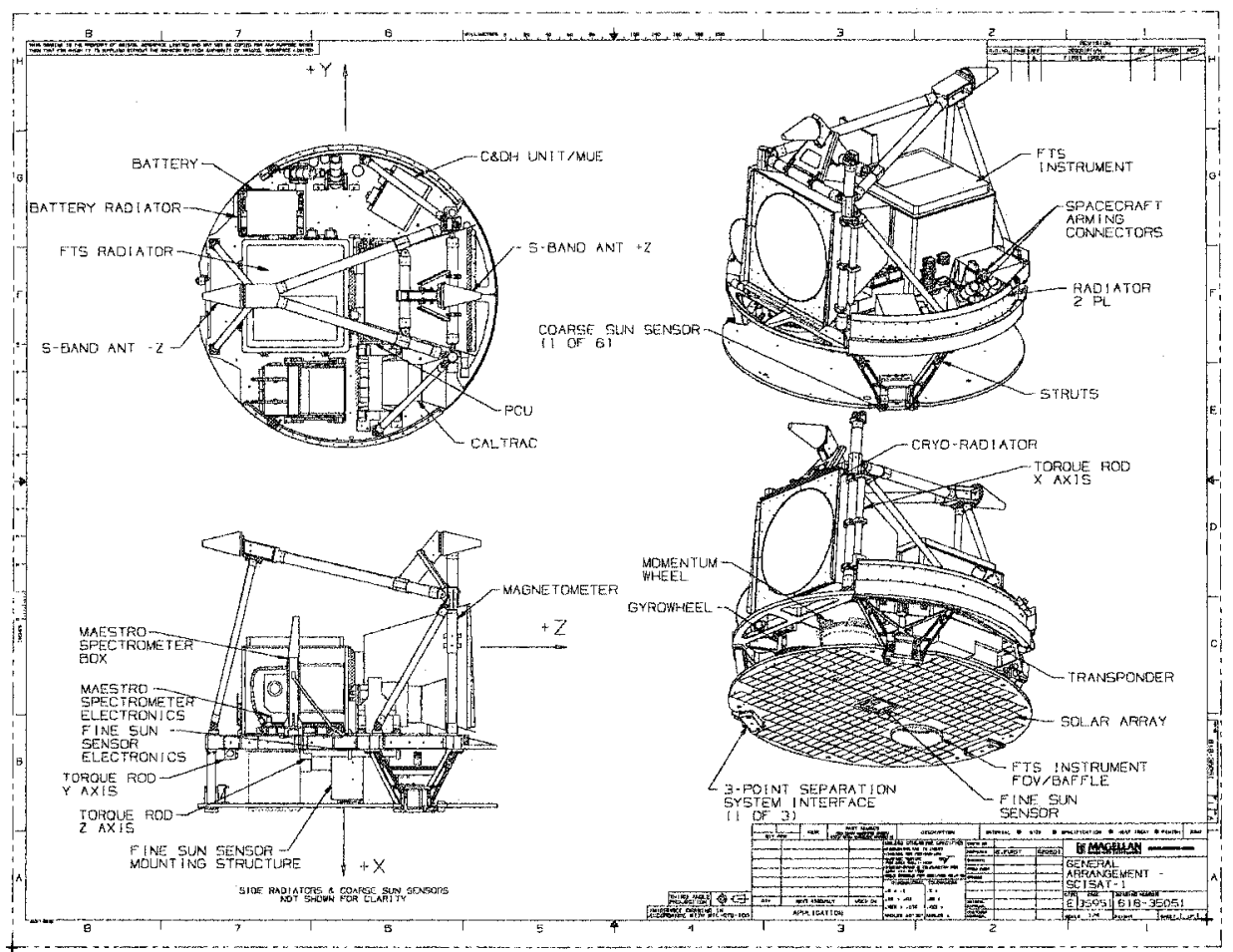

Figure 5.10 Multi-view schematic of Scisat-1 spacecraft. Refer to Annex 1 for larger diagram. (Courtesy Magellan Aerospace Corporation, 2002.) 
The mass budget includes some components which will not be required for the proposed solar sail mission. The ADCS uses torque rods to control the spacecraft which have a mass of $2 \mathrm{~kg}$. The torque rods would not be required for the solar sail mission since the sail will do all the spacecraft control as well as momentum dumping. Also within the ADCS are two types of momentum wheels, a classic momentum wheel with a mass of $6 \mathrm{~kg}$ and a Gyrowheel with a mass of $6.7 \mathrm{~kg}$. There are two wheels because the Gyrowheel is being flown as part of the mission objectives to test and evaluate it. Assuming that the Gyrowheel development progresses well, there would not be a need for the classic momentum wheel which would be a saving in mass.

Taking into account the components which are not required for the solar sail mission and the deletion of payload mass, the baseline total mass for the spacecraft becomes $102.8 \mathrm{~kg}$. Therefore, for the purpose of developing a total spacecraft mass which will include the solar sail, the baseline mass of $103 \mathrm{~kg}$ will be used for the core platform. In addition to the baseline mass, the mass of the solar sail, payload, stowage containers for the sail, pressurization system and control actuators must be added.

As previously determined, a mass of $21 \mathrm{~kg}$ will be used for the pressurization system and $6 \mathrm{~kg}$ will be estimated for the actuator and attachment points. Another addition to both the mass and power budget will be an additional attitude sensor for the ADCS. Scisat-1 uses a magnetometer, a Sun sensor and a star tracker to determine spacecraft orbit position and orientation. However, for the geomagnetic tail mission, the magnetometer would be ineffective and an additional sensor is required. The star tracker used on Scisat-1 determines only spacecraft orientation, therefore, to provide position information, either an additional star tracker needs to be installed or the star tracker needs 
to be switched for a star mapper. Since the worst case scenario is the addition of another star tracker, $4 \mathrm{~kg}$ will be added to the mass budget to compensate for the additional unit. For a saillet storage system, Bernasconi and Zurbuchen proposed that each saillet be stowed in a cylindrical container with a total mass for all four containers of $52.27 \mathrm{~kg}$. Given that the geomagnetic tail mission requires a larger sail, mass budget of $75 \mathrm{~kg}$ will be used for these containers. This mass will not be used to determine the size of the solar sails as it will be jettisoned prior to sail deployment. However, the mass of the storage containers will be approximated based on the volume of sail material and then added to the mass budget to determine a launch vehicle. Therefore, the total mass which will be used to determine the size of the solar sail, including the $20 \mathrm{~kg}$ payload, is $154 \mathrm{~kg}$.

Now it is possible to determine a size and mass of the sail required to propel a spacecraft mass of $154 \mathrm{~kg}$ given the performance metrics calculated in chapter 4. From table 4.2 , the performance metrics required for a real sail to fulfill the mission requirements are: $a_{0}=1.02716 \mathrm{~mm} / \mathrm{s}^{2}, \sigma=8.8035 \mathrm{~g} / \mathrm{m}^{2}$ and $\beta=0.1737$. Also, given that the total sail loading is equal to the sum of the spacecraft and the solar sail such that:

$$
\sigma=\sigma_{s p}+\sigma_{s s}=\frac{m_{s p}}{A_{s p}}+\frac{m_{s s}}{A_{s s}}
$$

however, given that $A_{s s}>>A_{s p}$, then $A_{s p}+A_{s s}=A_{s s}$ and,

$$
8.8035=\frac{m_{s p}+m_{s s}}{A_{s s}} .
$$

Also, from table 5.4 , the surface density of the sail material is $6.524 \mathrm{~g} / \mathrm{m}^{2}$ which means that $A_{s s} \times 6.524=m_{s s}$. Substituting this into (5.03) and solving for $m_{s s}$ gives a ratio of sail mass to spacecraft bus mass of $m_{s s}=2.862 \times m_{s p}$ or $m_{s s}=441 \mathrm{~kg}$ for a $m_{s p}$ of $154 \mathrm{~kg}$. 
These calculations represent the minimum sail mass and area required for the mission, with no reserves. To estimate some contingency reserves, consider that the performance metrics from table 4.2 are the minimum parameters required to meet the mission design criteria. If the spacecraft were at the extreme edge of the operational envelope, there would be no additional "thrust" to allow it to manoeuvre or change its position. Therefore, a reserve needs to be built into the design of the sail so that it can go to the desired operational limit of $100 \mathrm{R}_{\mathrm{E}}$ and still re-position itself along any axis. If a $5 \%$ reserve were applied to the sail loading, this would translate to a ratio of sail mass to spacecraft bus mass of $m_{s s}=3.547 \times m_{s p}$ or $m_{s s}=547 \mathrm{~kg}$. Therefore, the total sail area is $82,566 \mathrm{~m}^{2}$ and the performance metrics would become:

\begin{tabular}{|c|c|c|c|}
\hline & $\begin{array}{c}\text { Acceleration } \\
\mathrm{mm} / \mathrm{s}^{2}\end{array}$ & $\begin{array}{c}\text { Sail Loading } \sigma \\
\mathrm{g} / \mathrm{m}^{2}\end{array}$ & Lightness Number $\beta$ \\
\hline Ideal Sail & 0.9116 & 10.004 & 0.1528 \\
Real Sail & 1.02716 & 8.8035 & 0.1737 \\
Contingency & 1.0785 & 8.363 & 0.1828 \\
\hline
\end{tabular}

Table 5.7 Solar sail performance metrics for an ideal, real and a sail with some reserve thrust for exploring the Earth's geomagnetic tail.

With the total sail area calculated, it is now possible to determine basic sail dimensions which will allow for the sizing of the Nitrogen storage tanks as well as the sail storage canisters. Figure 5.11 depicts the saillet configuration.

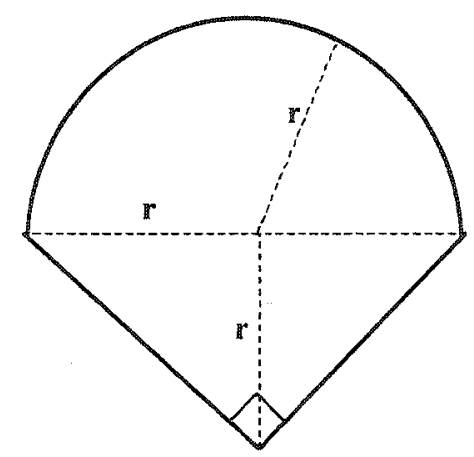

Figure 5.11 Diagram of saillet configuration. 
From the saillet geometry it is possible to derive the formula for sail area.

$$
A_{s s}=\frac{1}{2} \pi r^{2}+r^{2}
$$

Therefore, since there are four saillets comprising a total area of $82,566 \mathrm{~m}^{2}$, then each saillet has an effective surface area of $20,641.5 \mathrm{~m}^{2}$. Substituting this area into (5.04) yields $r=90 \mathrm{~m}$. This dimension can now be used to calculate the circumference of the saillet considering each side of the triangle is $\sqrt{2} r$ and the circumference of a half circle is $\pi \times r$. Therefore, the circumference is $538 \mathrm{~m}$ for each saillet.

From Bernasconi and Zurbuchen's article it is possible to derive the diameter of the saillet torus as being $0.15 \mathrm{~m}$ from the given parameters. ${ }^{121}$ Given that the proposed sail configuration will maintain the gas pressure inside the torus after the laminate material has cured, a diameter of $0.2 \mathrm{~m}$ for the torus will be used for calculating the volume of Nitrogen gas. Since the torus is simply a long tube, this gives a total volume of required Nitrogen gas of $17 \mathrm{~m}^{3}$. By using the ideal gas law: ${ }^{122}$

$$
p V=m R T
$$

where $p$ is the gas pressure, $V$ is the volume, $m$ is the mass of the gas, $R$ is the universal gas constant and $T$ is the temperature in Kelvin, it is possible to calculate the mass of Nitrogen required for each torus. Using Bernasconi and Zurbuchen's suggested $1.62 \mathrm{kPa}$, a rough temperature of $100 \mathrm{~K}$ for the geomagnetic tail environment and a value for $R$ of $0.2968 \mathrm{KJ} / \mathrm{Kg} \mathrm{K}$, the mass of the Nitrogen is $0.93 \mathrm{~kg}$. This means that there is an additional $3.8 \mathrm{~kg}$ of mass (four saillets) which will need to be added to the mass budget.

${ }^{121}$ Bernasconi and Zurbuchen, Table 3 p. 650.

${ }^{122}$ Richard E. Sonntag and Gordon Van Wylen, Introduction to Thermodynamics Classical and Statistical $2^{\text {nd }}$ Edition, John Wiley \& Sons Inc., USA, 1971.pp. 45-49. 
To determine the size of Nitrogen bottles required onboard the spacecraft, use the ideal gas law again based on $0.93 \mathrm{~kg}$ of Nitrogen and a nominal spacecraft bus temperature of $300 \mathrm{~K}$. Based on Bernasconi and Zurbuchen's assumption that the Nitrogen tanks would be operating at $21 \mathrm{MPa}$, the volume for each saillet is 4 litres. This means more mass needs to be budgeted for the slightly larger size Nitrogen tanks. Therefore, a contingency of $4 \mathrm{~kg}$ will need to be built into the pressurization system to allow for the additional mass of larger tanks and Nitrogen gas. Once this extra $4 \mathrm{~kg}$ has been added into the mass budget, the mass and size of the solar sail will need to be recalculated using (5.03). Adding in the additional $4 \mathrm{~kg}$ will give a spacecraft bus with a mass of $158 \mathrm{~kg}$ which then translates to a sail with a mass of $560 \mathrm{~kg}$ and an area of $84,530 \mathrm{~m}^{2}$.

As depicted in Table 5.6, the power budget for Scisat- 1 is $74.5 \mathrm{~W}$ with an average orbit power generation of $100 \mathrm{~W}$. Considering Scisat- 1 spends $36.5 \%$ of its time in eclipse, the maximum power generation is $156 \mathrm{~W}$ at the Earth's distance from the Sun. Since the geomagnetic tail mission is downstream from the Earth, maximum power generation by the solar array will obviously decrease slightly, however, the size of the solar array can most likely be increased to compensate. Therefore, for the purpose of producing a sample power budget, $156 \mathrm{~W}$ will be used as the average power generation, since the spacecraft will be continuously exposed to the Sun.

There will be a power savings in a couple of areas due to redundant equipment. Since the magnetometer and torque rods are not needed and use $1.5 \mathrm{~W}$, this will be a savings. As well, the Scisat-1 payload uses $30 \mathrm{~W}$ whereas a payload specific to exploring the geomagnetic tail will only use $10 \mathrm{~W}$. However, the actuators required to control the 
solar sails will use power, depending upon whether they are operated on a continuous basis or periodically. Also, the star tracker on Scisat- 1 is used only $20 \%$ of the time or $3.3 \mathrm{~W}$ per orbit. If the star trackers were run continuously on the proposed solar sail spacecraft, they would use $16.5 \mathrm{~W}$ each. ${ }^{123}$ Therefore, taking into account the power savings listed above, the Scisat-1 bus would consume $49.7 \mathrm{~W}$ of power without the star tracker. Then, if both star trackers were operated continuously, the power consumption would increase to $82.7 \mathrm{~W}$. Finally, using the maximum power generation of $156 \mathrm{~W}$ with a $25 \%$ loss factor $(117 \mathrm{~W})$, the remaining power could be used to power the actuators. Thus, the actuators must be chosen to fit within the power budget suggested below so that no additional power generation is required. The spacecraft mass and power budgets are detailed in table 5.8 below.

\begin{tabular}{|c|c|c|}
\hline Component & Mass (kg) & Power (W) \\
\hline Baseline Scisat-1 Bus & 103 & 56.2 \\
Payload & 20 & 10 \\
Pressurization System & 25 & 0 \\
Rotary Actuator Assembly & 6 & 34.3 \\
Additional Star Sensor & 4 & 16.5 \\
Sail Material and Structure & 560 & 0 \\
Sail Storage Containers & 75 & 0 \\
Total & $\mathbf{7 9 3} \mathbf{k g}$ & $\mathbb{1 1 7} \mathbf{W}$ \\
\hline
\end{tabular}

Table 5.8 Mass and power budgets for solar sail propelled Scisat-1 platform fitted with a solar sail propulsion system.

\subsection{From Launch to the Equilibrium Position}

Now that the mass budget for the proposed spacecraft has been established, it is possible to investigate launch vehicles in order to place the spacecraft into orbit. The

\footnotetext{
${ }^{123}$ E-mail from Mark Senez, Space Business Group, Bristol Aerospace Limited, 29 May 2002.
} 
minimum orbit altitude required for a solar sail spacecraft is $1000 \mathrm{~km}$, otherwise the atmospheric drag on the spacecraft will negate the acceleration derived from the solar radiation pressure. For an $800 \mathrm{~kg}$ class spacecraft there are a number of potential vehicles available with the capability of launching the satellite into a $1000 \mathrm{~km}$ orbit. It may also be possible to "piggyback" on a larger launch vehicle depending upon the available space.

As an example of the type of launcher that can handle the proposed solar sail spacecraft, consider the Rockot launch vehicle produced by Eurockot Launch Services. This launcher is a three-stage system capable of launching up to $1900 \mathrm{~kg}$ into low Earth orbit. The vehicle's reliability is extremely good with an almost $98 \%$ success rate. $^{124}$
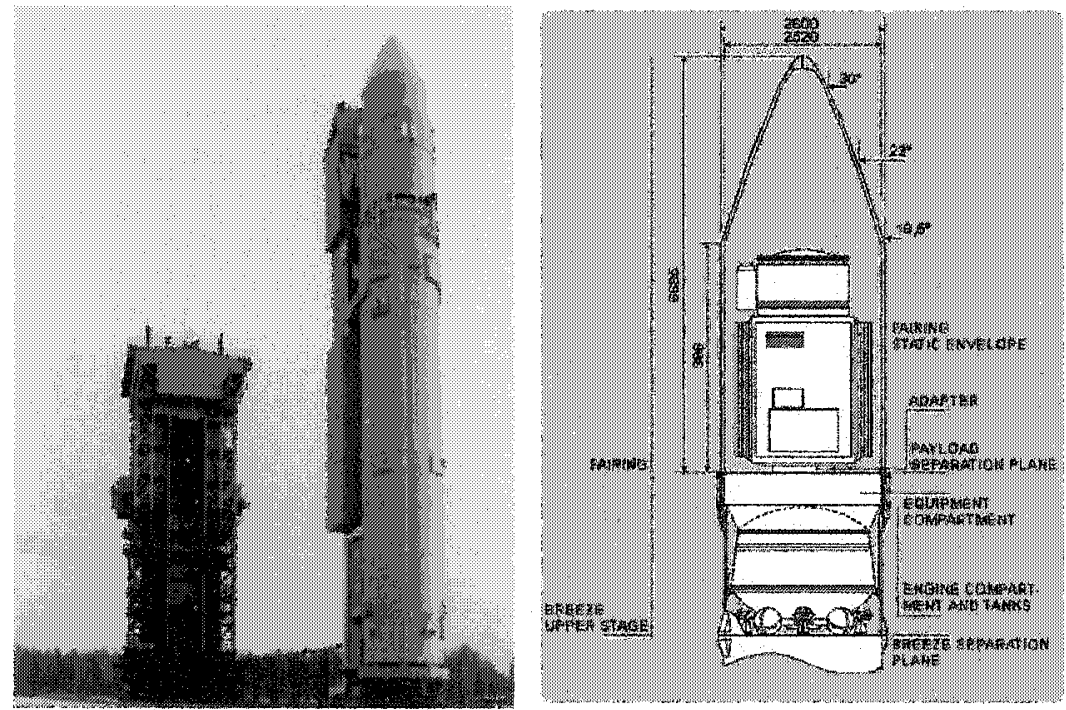

Figure 5.12 Rockot launch vehicle with service tower and schematic of payload compartment. (From Eurockot Launch Services, 2001.)

For a trajectory from a circular or elliptical orbit to the $L_{2}$ equilibrium point vicinity, it is important to reach the plane of the solar ecliptic. While it would be ideal to launch from the equator directly into a geosynchronous orbit and thus be very close to the

\footnotetext{
${ }^{124}$ Eurockot Launch Services, Rockot User's Guide, Issue 3 Rev. 1, 2001, p. 1-1.
} 
ecliptic plane, this is not possible given that Eurockot operates out of the Plesetsk Cosmodrome $\left(62.7^{\circ} \mathrm{N}\right)$ or the Baikonur Cosmodrome $\left(45.6^{\circ} \mathrm{N}\right)$. Therefore, Baikonur, being the most southerly of the two locations, would be the preferred launch site. Once in orbit, the solar sail can perform a cranking manoeuvre to reduce the orbit inclination. The performance data for the Rockot launcher is depicted below in figure 5.13.
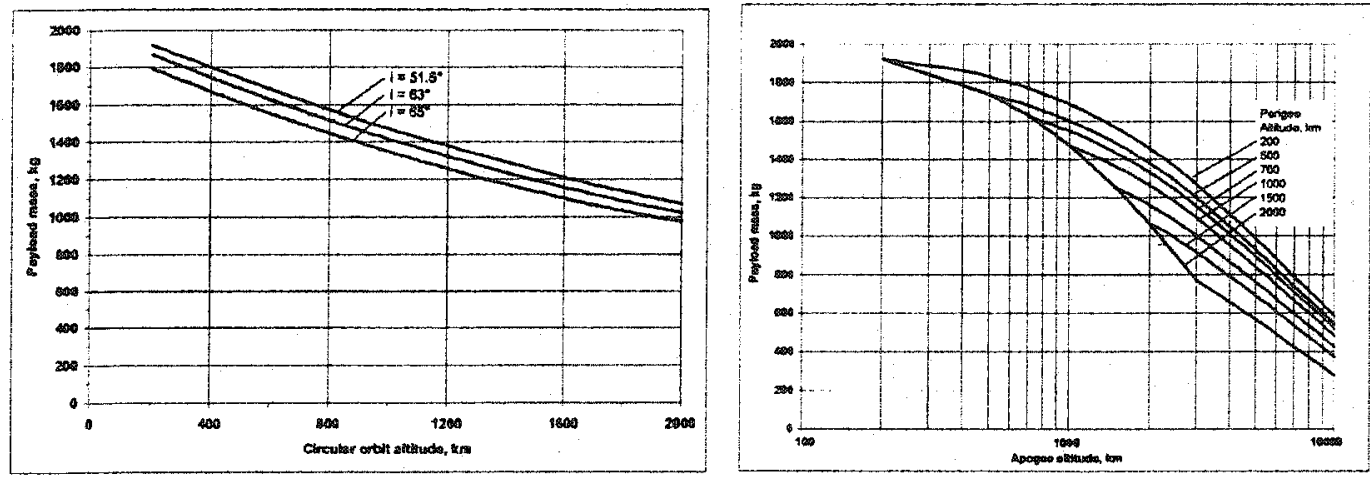

Figure 5.13 Performance data for the Rockot launch vehicle for circular and elliptical orbits. Note that this data is for launch from Baikonur Cosmodrome. (From Eurockot Launch Services, 2001.)

From figure 5.13, it is possible to determine the maximum altitude to which the Rockot launch vehicle can propel an $800 \mathrm{~kg}$ class spacecraft. For a circular orbit, the maximum altitude for a $51.6^{\circ}$ inclination is well over $2000 \mathrm{~km}$ while for an elliptical orbit there is a range of apogee altitudes depending on the desired perigee height. If a minimum perigee height of $2000 \mathrm{~km}$ is used, this corresponds to an apogee distance of approximately $2800 \mathrm{~km}$. It is important to note that the Rockot launch vehicle is capable of launching a $1000 \mathrm{~kg}$ class spacecraft into a $2000 \mathrm{~km}$ circular orbit should the proposed mass budget increase due to design revisions.

Once launched into the desired orbit, sail deployment would take place so that the spacecraft can begin a cranking procedure to reduce the orbit inclination. The cranking procedure consists of the sail being oriented during one half of the orbit so that the solar 
radiation pressure force is directed towards the solar ecliptic. During the second half of the orbit the sail orientation must be changed in the opposite direction in order that the solar radiation pressure force continues to reduce the orbit inclination. After a number of orbits, the spacecraft will slowly align with the plane of the ecliptic.

For the solar sail spacecraft to travel from the Earth to the equilibrium contours, Hur and Bryson have stated that a combined local optimization and shooting method needs to be applied so that the spacecraft arrives at the desired location with zero velocity. ${ }^{125}$ Local optimization is performed by maximizing the time rate of change of the energy variable because the concept is to increase the orbital energy as quickly as possible to reach Earth escape velocity. As the energy increases, the solar sail orbit spirals outward as depicted in figure 5.14 .
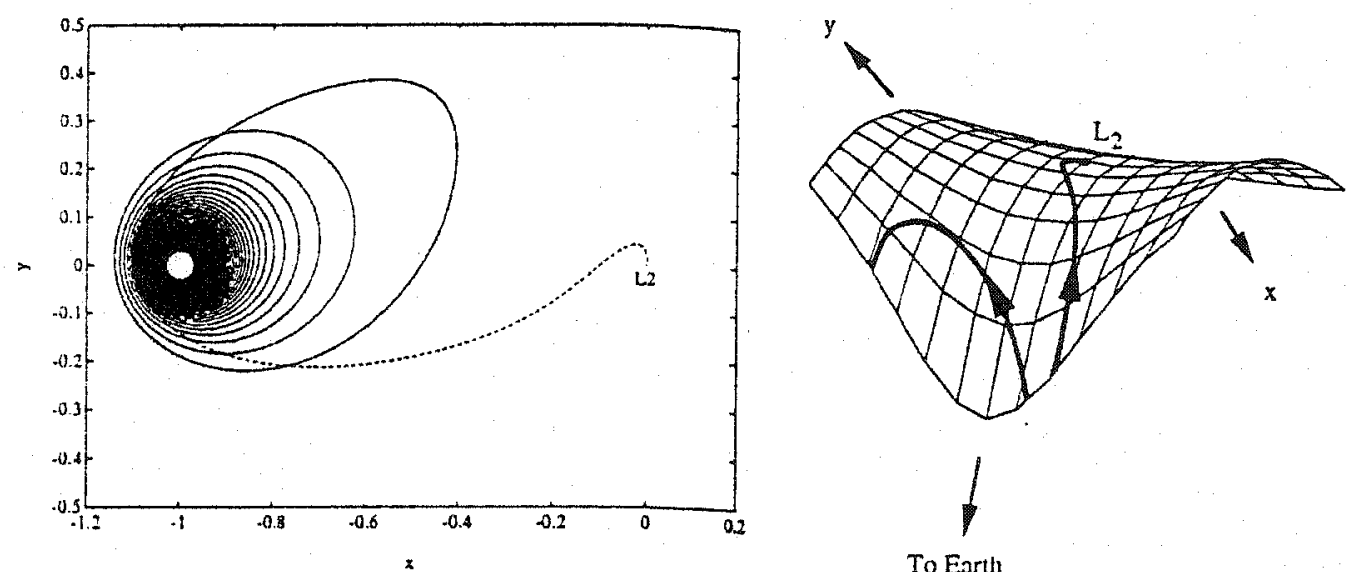

Figure 5.14 Solar sail trajectory plot to reach $\mathrm{L}_{2}$ point using combined local optimization and single shooting method. (From Hur \& Bryson, 1992.)

As the spacecraft travels towards the Sun, each saillet is turned edge on towards it so that there is no solar radiation pressure force generated. As the spacecraft begins to move away from the Sun, the saillets are turned to face it so that the radiation pressure force can increase the orbital energy. Once the orbital energy has increased to the point 
of Earth escape, a single "backward shooting method [is] used to obtain the minimum time transfer segment from the last perigee to the $\mathrm{L}_{2}$ point." ${ }^{.126}$ The shooting method must be performed so that the spacecraft arrives at its destination with zero velocity, otherwise it may overshoot or undershoot the position and require additional steering commands to manoeuvre it onto the equilibrium contours.

Hur and Bryson's work resulted in a transfer time from a geosynchronous orbit to the $L_{2}$ point of 2.01 years using a characteristic acceleration of $0.1 \mathrm{~mm} / \mathrm{s}^{2}$. The transition segment from the last perigee to the equilibrium point occupied approximately $20 \%$ of the total time. During this portion of the transfer, the sail was turned edge-on to the Sun, generating almost zero force so that the primary forces were the gravitational and centrifugal ones. ${ }^{127}$ The last portion of the paper suggested a transfer to an equilibrium point $15.6 \%$ closer to the Earth which would decrease the transfer time to 1.86 years. ${ }^{128}$

As a means of comparing Hur and Bryson's work with the geomagnetic tail solar sail performance metrics, consider that they used a characteristic acceleration of 0.1 $\mathrm{mm} / \mathrm{s}^{2}$, while this thesis proposes a solar sail which can generate just over $1 \mathrm{~mm} / \mathrm{s}^{2}$. Therefore, the time required to reach an Earth escape velocity will be greatly reduced because the sail can accelerate more quickly. However, it is doubtful that the proposed spacecraft will start from a geosynchronous orbit unless it is able to piggyback on a larger launch vehicle than the Rockot. Therefore, more time will be required to increase the orbital energy from the lower altitude until an Earth escape velocity may be reached. It

\footnotetext{
${ }^{125}$ Sun H. Hur and Arthur E. Bryson Jr., "Minimum Time Solar Sailing from Geosynchronous Orbit to the Sun-Earth $L_{2}$ Point", AIAA-92-4657, AAS/AIAA Astrodynamics Conference, August 1992, p. 540. ${ }^{126}$ Ibid.

${ }^{127}$ Ibid. p. 541.

${ }^{128}$ Ibid. p. 542.
} 
may be that the time saved due to the higher performance solar sail will balance out the extra time required to travel from the lower altitude.

The time cited by Hur and Bryson as required from the last perigee to the equilibrium position, some $15.6 \%$ closer to the Earth, will probably be the same for the mission proposed in this thesis, since the sail is practically "turned off" during this segment of the transfer. This portion of the transfer time is approximately 120 days. While there is no intention to delve into this subject in great detail, a qualitative comparison suggests that the transfer time from an Earth orbit of $2000 \mathrm{~km}$ to an equilibrium point $15.6 \%$ closer to the Earth than the $\mathrm{L}_{2}$ point could be less than 1.86 years given that the characteristic acceleration of the solar sail is greater than Hur and Bryson's example by a factor of ten.

\subsection{Traveling within the Earth's Geomagnetic Tail}

Once the spacecraft has arrived at a preliminary equilibrium point, it must conduct station keeping manoeuvres to maintain its position. Therefore, the Attitude Determination and Control System (ADCS) requires a specific suite of sensors to determine both position and orientation of the spacecraft. The Scisat-1 platform has a combination of both course and fine Sun sensors to determine its position relative to the Sun and a star tracker to determine its orientation. All of these instruments would be beneficial for the geomagnetic tail mission since it will be fairly important for the spacecraft to maintain an accurate position in order to measure the magnetic fields and

particle interactions. However, these two sensors alone will not provide the ADCS all the information required to maintain an accurate position. 
As previously mentioned, a second star tracker can be added to get a second star vector and, therefore, position data. Another technique which could be employed to determine a distance from the Earth is called Doppler Tone Ranging, where tones are sent from the ground station to the spacecraft and the Doppler shift is measured. By measuring the Doppler shift, the ADCS is able to calculate the distance from the Earth as well as relative velocity.

The ADCS will be able to determine the spacecraft position relative to the Sun and Earth using the suite of instruments described above. Although not an absolute requirement, it would be desirable if the ADCS had the ability to conduct not only station keeping manoeuvres but also navigation manoeuvres to reach different positions which would be directed from the ground station. This would simplify the navigation commands being uplinked to the spacecraft, since the on-board navigation computer would be able to carry out the command autonomously.

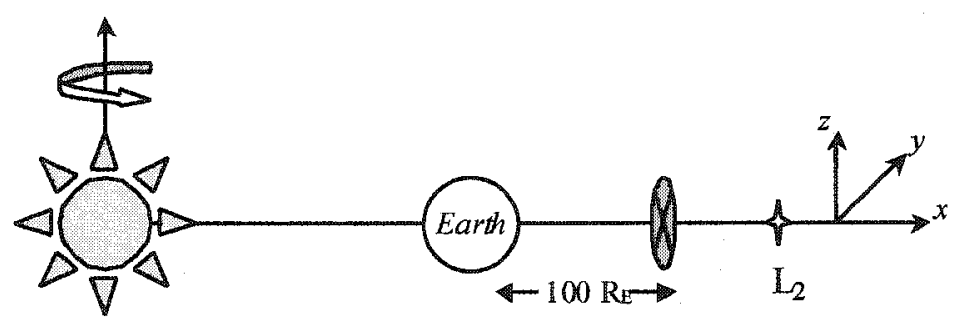

Figure 5.15 Hypothetical set up of a solar sail operating at $100 \mathrm{R}_{\mathrm{E}}$ in line with Sun and Earth.

In order for the solar sail spacecraft to travel to various points within the Earth's geomagnetic tail, a hypothetical situation, as depicted in figure 5.15, will be considered where the spacecraft is positioned in line with both the Sun and Earth at the operational design limit of $100 \mathrm{R}_{\mathrm{E}}$. 
At this position the sail would not be generating its maximum force, since the saillets would be slightly angled towards each other to maintain equilibrium. To travel along the $x$ axis towards the $\mathrm{L}_{2}$ point, the sail must generate more force to counteract the gravitational forces of the Sun and Earth. Therefore, the saillets would turn to create a flat sail that was perpendicular to the sunlight so that the maximum solar radiation pressure force was being generated. As the sail traveled along the $x$ axis towards the $\mathrm{L}_{2}$ point, small station keeping manoeuvres would be required to maintain the Sun-Earth line. Upon approaching a new destination closer to the $\mathrm{L}_{2}$ point, the saillets would angle inwards again to reduce the force generated by the sail. The spacecraft would then decelerate and, upon reaching the new position, the saillets would be turned to a specific angle required to maintain equilibrium. Note that the new angle is greater than the original one at $100 \mathrm{R}_{\mathrm{E}}$, since the closer the spacecraft travels towards the $\mathrm{L}_{2}$ point, the less solar radiation pressure force is required to maintain equilibrium.

For the spacecraft to travel in the opposite direction along the $x$ axis, the saillets would turn so that they were edge-on with the sunlight and, therefore, not generating any force. Gravitational forces from the Sun and Earth would take over, pulling the spacecraft towards them. As the solar sail approached the new position closer to the Earth, the saillets would turn so that the maximum force was being generated to decelerate the spacecraft. Upon reaching the new destination, again the saillets would be turned to a specific angle required to maintain equilibrium.

For the spacecraft to travel in either the $y$ axis or $z$ axis direction, the entire sail will need to be turned to generate a transverse force. By turning one saillet edge-on with the Sun, a torque will be generated from the opposing one as it generates solar radiation 
pressure force. The torque will rotate the orientation of the sail so that a transverse force will be generated in the desired direction of travel as depicted in figure 5.16. The sail angle used to generate the transverse force must be sufficient so that the spacecraft can travel to the new destination, slow down and then maintain the position.
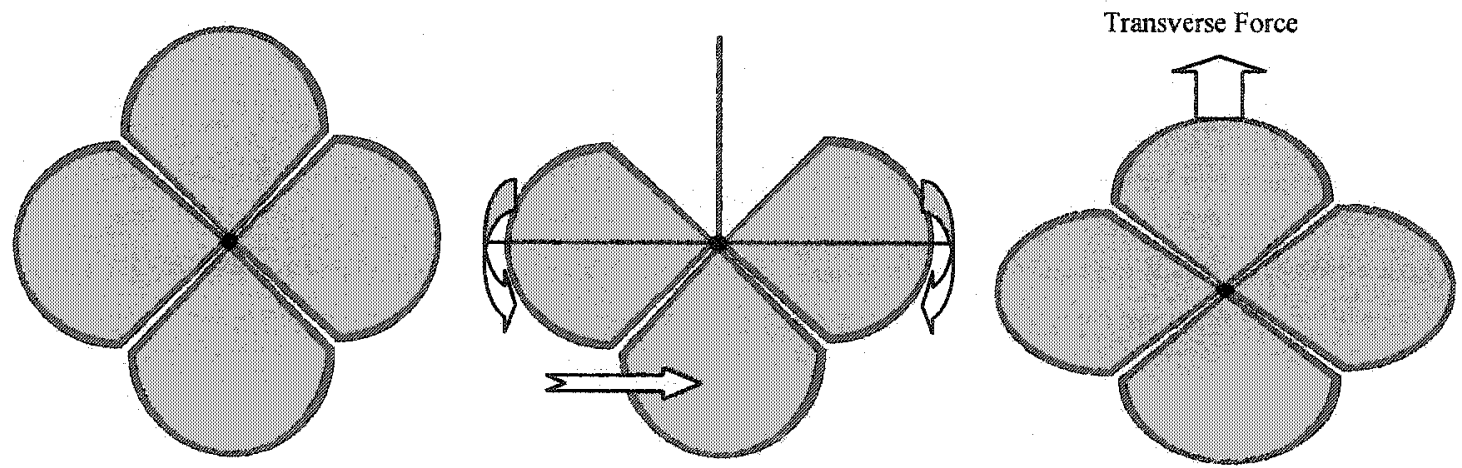

Figure 5.16 Steering manoeuvre used to generate transverse force by rotating sail orientation.

To maintain equilibrium at a new position along the $y$ or $z$ axes, the solar sail will use a combination of overall sail angle as well as saillet angles to regulate the solar radiation pressure force so that equilibrium is maintained.

For the proposed mission to the Earth's geomagnetic tail, a phenomenon which will affect the mission is the Earth's umbra and penumbra. Since the solar sail derives its "thrust" from the Sun's radiation, it will be impossible to position the spacecraft within the umbra and traveling within the penumbra will mean a degradation of solar sail output due to the partially eclipsed Sun.

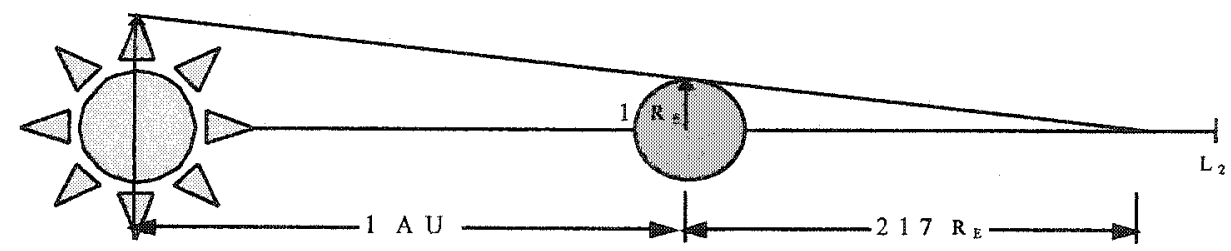

Figure 5.17 Depiction of Earth's umbra. Note that the shadow almost reaches the $L_{2}$ point. 
The Earth's shadow is a major mission design consideration given that it extends behind the Earth by $216 R_{E}$ from the Earth's surface. Since one of the mission objectives outlined in table 2.1 is to reach $100 R_{E}$ along the Sun-Earth line, it will be impossible to maintain this position due to the shadow. The only way to carry out any mission objectives within the umbra is to start well outside of it and then accelerate and travel through to the other side. Since the diameter of the shadow at $100 R_{E}$ is $5932 \mathrm{~km}$, the spacecraft control strategy must compensate for the fact that while traveling through the umbra the solar sail force is nonexistent and the gravitational forces of the Sun and Earth will pull the spacecraft towards them. Therefore, the operational envelope will need careful consideration to ensure that the spacecraft stays well inside of it while performing one of these manoeuvres.

The Earth's penumbra will also play a role when defining the spacecraft control strategy. Since the force generated by the solar sail is directly related to the amount of solar radiation pressure it encounters, any partial eclipsing of the Sun by the Earth will result in the degradation of that force. The geometry of the penumbra is depicted in figure 5.18 .

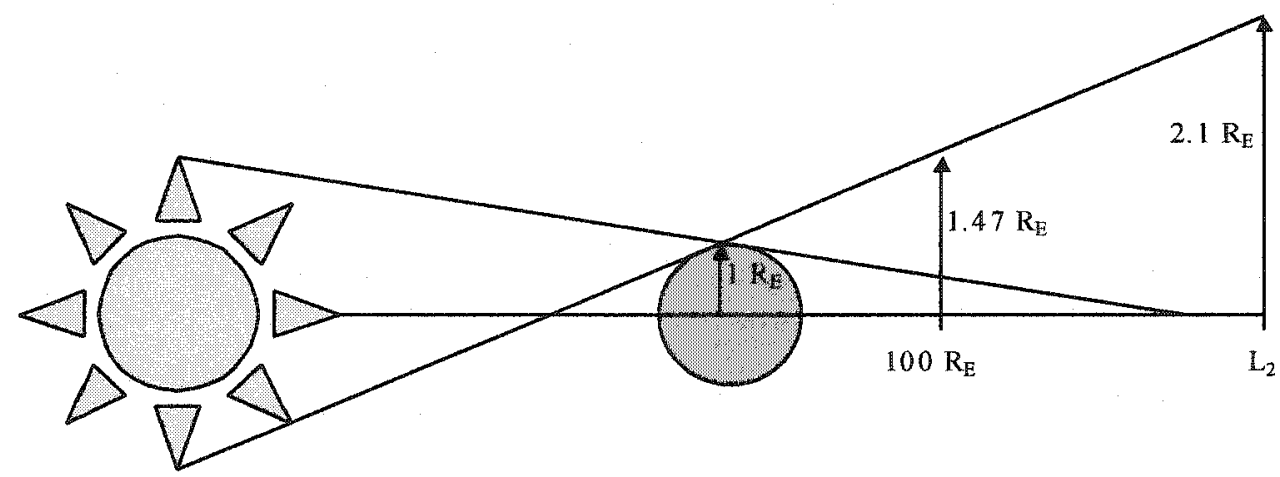

Figure 5.18 Depiction of Earth's penumbra. Note that the distances are radii of a circle. 
When dealing with the partial eclipse region, the control strategy could be the same as with the umbra, meaning the spacecraft will simply transit through and not attempt to position itself within the penumbra. However, there may be sufficient solar radiation pressure available, depending upon the required solar sail force, to maintain equilibrium. For example at $100 \mathrm{R}_{\mathrm{E}}$, which is the operational limit of the proposed solar sail, any incursion into the penumbra would degrade the force generated by the sail and the spacecraft would then be pulled towards the Earth. Therefore, a limiting geometry for the mission at $100 \mathrm{R}_{\mathrm{E}}$ would be $1.47 \mathrm{R}_{\mathrm{E}}$, and any travel within this distance must be transitory. However, out by the $\mathrm{L}_{2}$ point, the amount of solar sail force required to maintain equilibrium is very little, therefore, the reduced solar radiation pressure may not cause any problem at all. Further investigation into the limits of operating within the penumbra would be required and these limits would need to be incorporated into any detailed navigation strategy.

It is important to note that the work by both Forward and McInnes does not take into account the effect of the Earth's shadow on the equilibrium contours between the Earth and the $\mathrm{L}_{2}$ equilibrium point. The equilibrium surfaces would have an area of "No Solutions" corresponding to the region occupied by the umbra. As well, the penumbra region would need to be modified to show the reduction of the solar radiation pressure force proportional to the increase of the solar eclipse. 


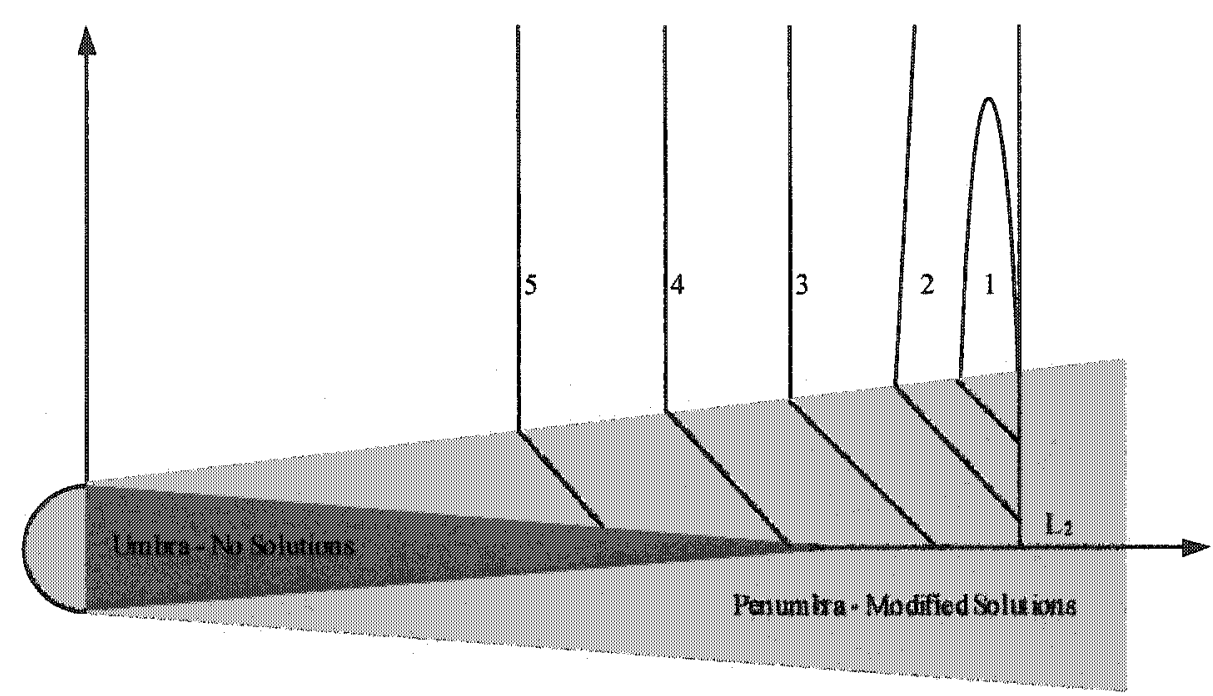

Figure 5.19 Hypothetical equilibrium contours from Earth to the $L_{2}$ point with modifications for the umbra and penumbra regions.

Figure 5.19 depicts a hypothetical representation of the equilibrium contours within the Earth's penumbra such that the contours angle towards the $\mathrm{L}_{2}$ point due to the effect of the solar eclipse. The exact shape of these contours has not been proven mathematically and is, therefore, unknown at this time.

\subsection{Summary}

A solar sail propelled spacecraft has been further developed using the performance metrics described in chapter 4 . The sail configuration was explored with a preferred design suggested using four cloverleaf shaped saillets. This configuration gives the solar sail the required flexibility to be able to carry out the mission objectives to explore the geomagnetic tail. Once the configuration was decided, the total spacecraft mass was calculated based on assumptions made for the sail material and support structure, which resulted in a mass budget for a $800 \mathrm{~kg}$ class satellite.

Once a spacecraft mass budget was developed, the method for launching the satellite was explored and a navigation plan detailed for getting from an approximate 
$2000 \mathrm{~km}$ orbit with an inclination of $51.6^{\circ}$ to an equilibrium position inside the geomagnetic tail. Finally a control strategy was developed to suggest how the solar sail would manoeuvre inside the operational envelope in order to accomplish the mission objectives. The Earth's umbra and penumbra were also discussed, since they would have a direct impact on the mission objectives given that the sail cannot operate within a shadow. 


\section{Chapter 6 - Conclusions and Recommendations}

The objective of this thesis was to investigate the possibility of positioning a scientific payload permanently in the Earth's geomagnetic tail using solar sail propulsion to counteract the gravitational forces of the Sun and Earth. After a brief survey of the composition of the magnetosphere and geomagnetic tail, mission objectives were established in the form of distances which needed to be achieved in order to conduct the required investigation into magnetospheric particle dynamics. Following this, solar sail technology was investigated with specific interest paid to solar sailing in a restricted three-body system. The result of this investigation led to the establishment of sail performance metrics which would be required to meet the mission objectives. These performance metrics were considered to represent a moderate performance solar sail but one which was very achievable by current materials technology.

Following the development of the solar sail performance metrics, various aspects of the spacecraft design were discussed as a means to develop an overall spacecraft mass budget. Once this was completed, a potential launch strategy was investigated, including a method for traveling from a $2000 \mathrm{~km}$ by $2700 \mathrm{~km}$ elliptical orbit to an equilibrium position within the geomagnetic tail. Finally, control and navigation strategies were discussed, including the issue of the Earth's shadow and how it would affect the mission.

Perhaps one of the most significant discoveries of this thesis involved the mass of the solar sail materials. Since the overall spacecraft mass is inextricably connected to the mass of the solar sail materials and structural members, the total mass grew significantly 
as more material was introduced to attain the performance metrics. While the sail material mass per unit area appeared to be fairly low, the rigidizable laminate surface density was quite high. In fact, even though the rigidizable laminate only occupied $3 \%$ of the total sail area, its surface density contributed significantly to the total mass of the saillets. Given that the information regarding this laminate material was gathered from the article by Bernasconi and Zurbuchen, it is recommended that any future investigations into this mission must focus on a more thorough analysis of the forces and torques being applied to the saillets and how their design is impacted. Perhaps the surface density of the rigidizable laminate can be reduced, which would reduce the overall mass of the spacecraft.

One of the original concepts for this thesis was to design a spacecraft which could be launched from a Pegasus XL launch vehicle. Unfortunately, the possibility of this became increasingly impossible as the spacecraft design progressed. The suggestion to use the Scisat-1 platform as a central control hub is valid since the research and development has already been invested. However, once certain extraneous components were removed from the Scisat-1 mass budget and solar sail specific ones added, the platform's mass increased to roughly $158 \mathrm{~kg}$. For a Pegasus XL launch vehicle to boost a spacecraft to a $1000 \mathrm{~km}$ circular orbit, the limiting mass is $250 \mathrm{~kg}$. Therefore, it became obvious that the Pegasus XL was not going to be the launch vehicle. With a $158 \mathrm{~kg}$ payload, the solar sail materials required to meet a characteristic acceleration of just over $1 \mathrm{~mm} / \mathrm{s}^{2}$ had a mass of $560 \mathrm{~kg}$. The combination of the payload and sail masses put the spacecraft into the $800 \mathrm{~kg}$ category, which suggested a Rockot launch vehicle or a piggyback scenario on a larger launcher. As one possible solution, the Rockot was 
discussed, but further investigation could be carried out to determine the optimum launch strategy.

An article by Hur and Bryson was used to demonstrate the strategy for the solar sail to optimize its escape trajectory and reach the equilibrium point. The intention here was not to go into great detail for the timeline of the proposed mission, but to show that work has been done in this field and that the local optimization and shooting method does in fact work. Again, the next level of investigation would need to recreate the method documented in the article with the proposed characteristic acceleration to determine a better idea of the timeline. Also, consideration must be made for the possibility that the spacecraft could be launched into an inclined orbit and will need to conduct a cranking manoeuvre to achieve the solar ecliptic plane before attempting an Earth escape optimization trajectory.

Another area which would require an extensive amount of investigation is the navigation and spacecraft control strategy. Some considerations for this strategy were given in chapter 5 but, unfortunately, they barely scratched the surface of the topic. The greatest effort will need to be directed towards a method for the spacecraft to travel from one position to another without overshooting the operational envelope. Consider that the spacecraft has been directed to travel to a point along the $z$ axis. First it will rotate a saillet so that a torque is induced to change the orientation of the entire sail in the direction of travel. The amount of sail angle will depend somewhat upon the ultimate destination but also on the fact that the maximum transverse force occurs at $35^{\circ}$. Once oriented correctly, the sail begins to accelerate along the $z$ axis towards the new position. Now the problem becomes one of timing. When does the sail re-orient itself so as to not 
overshoot the intended target position? Also, does the new position require more or less thrust from the solar sail since this will also factor into the timing strategy. Finally, how does the sail steer itself into position? Does it simply turn all saillets edge-on to the Sun so that a combination of gravitational forces and the centrifugal force will slow it down or does it need to re-orient itself to decelerate? These questions need to be answered to formulate a more comprehensive navigation strategy as well as some control laws.

The issue of the Earth's shadow was also investigated to show that it will have an impact on the solar sail as it travels in the geomagnetic tail. Any operation other than transiting through the umbra will be impossible, since the sail would not be able to generate any thrust. This does not, however, jeopardize the mission objectives, since the spacecraft can still operate well outside of the umbra and penumbra. In fact, the umbra and penumbra will have little effect on the mission objectives since the geomagnetic tail has a radius of $27 R_{E}$. and the umbra is less than $1 / 2 R_{E}$ at the operational limit of $100 R_{E}$ anti-sunward of the Earth. Most likely the spacecraft will be operating near the magnetopause to investigate the particle and magnetic field dynamics as the solar wind interacts with the outermost layer of the magnetosphere.

A solar sail propelled spacecraft may one day become a reality. There is certainly enough interest in the space community to develop innovative propulsion systems, given the limits of chemical propulsion. As well, solar sailing offers a much wider range of potential mission applications than chemical propulsion, since the solar sail is continuously generating thrust and is only limited by the life expectancy of the sail material. One of the mission applications ideally suited to this type of propulsion is a long-term exploration of the Earth's geomagnetic tail to discover the dynamics of ionized 
particles and how they interact within the magnetosphere. Since Canada has a great deal of interest in this area of research, this investigation would complement Canada's current program as outlined in Space Weather in Canada: A Plan of Action Under LTSP III. With the constant advances being made with materials technology and the innovative sail configurations being proposed, the opportunity to forge ahead in the field of solar sail propulsion is now. Canada could be at the forefront of this technology with an aggressive investment of some time and resources. 


\section{Annex 1-Scisat-1 Schematic}

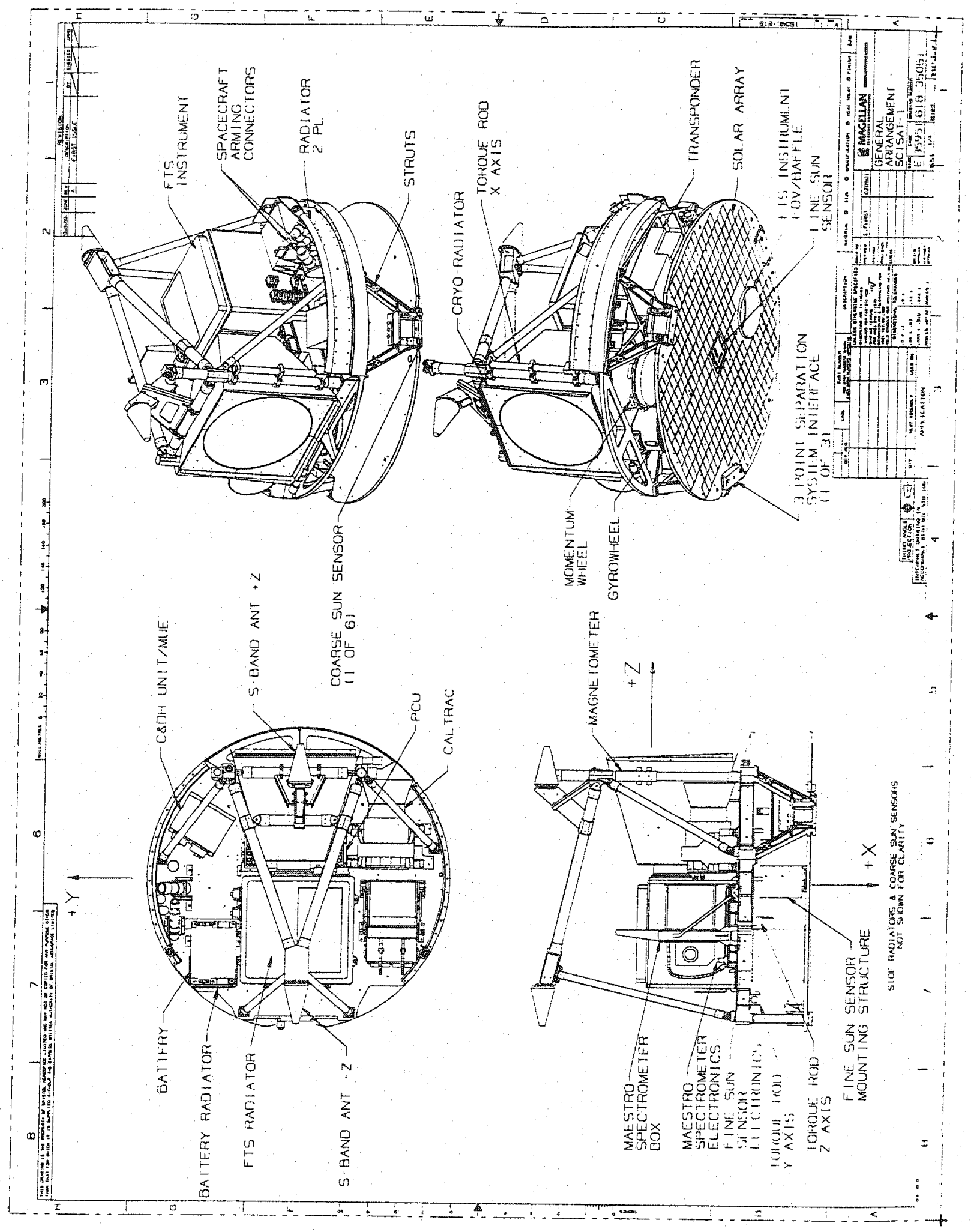




\section{Bibliography}

Bernasconi, Marco C., Zurbuchen, Thomas, "Lobed Solar Sails for a Small Mission to the Asteroids", Acta Astronautica Vol. 35, Supplement, 1995, pp. 645-655.

Blomquist, Richard Stuart, "Design Study of a Solid-State Heliogyro Solar Sail", Master of Science Thesis, Massachusetts Institute of Technology, August 1990.

Carroll, Kieran A., "POLARES Solar Sail Feasibility Study", Dynacon TM 39-306/04-1, Dynacon Enterprises Ltd., October 8, 1993.

Carroll, Kieran A., Hughes, Peter C. \& Simmons, Donald F., "Solar Sail Applications, Dynamics and Control Study," Dynacon Report 29-501/01-1, Dynacon Enterprises Ltd., March 1995.

Carroll, Kieran A., "Economical Planetary Space Science Using Small Solar Sail Propelled Spacecraft", Proceedings of the $10^{\text {th }}$ Conference on Astronautics, Canadian Aeronautics and Space Institute, Ottawa, October 1998.

Cassenti, B.N., Matloff, G.L. and Strobl, J., "The Structural Response and Stability of Interstellar Solar Sails", Journal of The British Interplanetary Society, Vol. 49, 1996, pp. $345-350$.

Dornheim, Michael A., "Inflatable Structures Taking Flight", Aviation Week and Space Technology, Vol. 150 No. 4, 25 Jan 99, pp. 60-62.

Eurockcot Launch Services, Rockot User's Guide, Issue 3, Rev. 1, 2001.

Fekete, Thomas A., Sackett, Lester L. \& von Flotow, Andreas H., "Trajectory Design for Solar Sailing from Low-Earth Orbit to the Moon", AAS-92-184, AIAA Spaceflight Mechanics Meeting, February 1992, pp. 1083-1094.

Fieseler, Paul D., "A Method For Solar Sailing in a Low Earth Orbit", Acta Astronautica Vol. 43, No. 9-10, 1998, pp. 531-541.

Forward, Robert L., "Statite: A Spacecraft That Does Not Orbit," Journal of Spacecraft and Rockets, Vol. 28, No. 5, 1991, pp. 606-611.

Forward, Robert L., "Light-levitated Geostationary Cylindrical Orbits," Journal of Astronautical Sciences, Vol. 29, No. 1, 1981, pp. 73-80.

Forward, Robert L., "Light-levitated Geostationary Cylindrical Orbits Using Perforated Light Sails," Journal of Astronautical Sciences, Vol. 32, No. 2, 1984, pp. 221-226.

Forward, Robert L., "Light-levitated Geostationary Cylindrical Orbits: Correction and Expansion," Journal of Astronautical Sciences, Vol. 38, No. 3, 1990, pp. 335-353. 
Genta, Giancarlo, \& Brusa, Eugenio, "The Parachute Sail with Hydrostatic Beam: A New Concept for Solar Sailing", Acta Astronautica, Vol. 44, No. 2-4, 1999, pp. 133-140.

Genta, Giancarlo, \& Brusa, Eugenio, "The Aurora Project: A New Sail Layout", Acta Astronautica, Vol. 44, No. 2-4, 1999, pp. 141-146.

Glotova, M. Yu \& Shvartsburg, A.A., "Geocentric Synchronous Halos for a Solar Sail", Soviet Physics Doklady, Vol. 38, No. 12, December 1993, pp. 499-504.

Gombosi, Tamas I., Physics of the Space Environment, Cambridge University Press, U.S.A., 1998.

Hur, Sun H. \& Bryson, Arthur E., "Minimum Time Solar Sailing from Geosynchronous Orbit to the Sun-Earth $L_{2}$ Point", AIAA-92-4657, AAS/AIAA Astrodynamics Conference, August 1992, pp. 538-543.

Jack C. and Welch C.S., "Solar Kites: Small Solar Sails with No Moving Parts", Acta Astronautica, Vol. 40 No. 2-8, 1997, pp. 137-142.

Kivelson, Margaret G. and Russell, Christopher T., Introduction to Space Physics, Cambridge University Press, USA, 1995.

Koblik, V.V., Polyakhova, E.N., Sokolov, L.L \& Shmyrov, A.S., "Controlled Solar Sailing Transfer Flights into Near-Sun Orbits under Restrictions on Sail Temperature", Cosmic Research, Vol. 34, No. 6, 1996, pp. 572-578.

Larson, Wiley J. and Wertz, James R., Space Mission Analysis and Design Second Edition, Microcosm Inc., USA and Kluwer Academic Publishers, The Netherlands, 1992.

Leipold, M., Borg, E., Lingner, S., Pabsch, A., Sachs, R., Seboldt, W., "Mercury Orbiter with a Solar Sail Spacecraft," IAA International Conference on Low-Cost Planetary Missions, Laurel, Maryland, April 1994, Acta Astronautica Vol. 35, Supplement, 1995, pp. 635-644.

Leipold, M., "To the Sun and Pluto with Solar Sails and Micro-Sciencecraft," $3^{\text {rd }}$ LAA International Conference on Low-Cost Planetary Missions, Pasadena, California, 27 April - I May 1998, Acta Astronautica Vol. 45, Nos. 4-9, 1999, pp. 549-555.

Leipold, M., Garner, C.E., Freeland, R., Herrmann, A., Noca, M., Pagel, G., Seboldt, W., Sprague, G., Unckenbold, W., "ODISSEE - A Proposal for Demonstration of a Solar Sail in Earth Orbit," $3^{\text {rd }}$ IAA International Conference on Low-Cost Planetary Missions, Pasadena, California, 27 April-1 May 1998, Acta Astronautica Vol. 45, Nos. 4-9, 1999, pp. 557-566.

Leipold, M., Seboldt, W., Lingner, S., Borg, E., Herrmann, A., Pabsch, A., Wagner, O., Brückner, J., "Mercury Sun-Synchronous Polar Orbiter with a Solar Sail", Acta Astronautica, Vol. 39, No. 1-4, 1996, pp. 143-151. 
Leipold, M., \& Wagner, O., "Solar Photonic Assist Trajectory Design for Solar Sail Missions to the Outer Solar System and Beyond", AAS/GSFC $13^{\text {th }}$ International Symposium on Space Flight Dynamics, Goddard Space Flight Center, Greenbelt, Maryland, May 11-15, 1998, pp. 1035-1045.

Leipold, M., Lingner, S., Borg, E., and Brüeckner, J., "Mercury Imaging from a SunSynchronous Solar Sail Orbiter", Journal of the British Interplanetary Society, Vol. 49, 1996, pp. 105-112.

Leipold, M., \& Wagner, O., "Mercury Sun-Synchronous Polar Orbits Using Solar Sail Propulsion", Journal of Guidance, Control and Dynamics, Vol. 19, No. 6, 1996, pp. 1337-1341.

London, Howard S., "Some Exact Solutions of the Equations of Motion of a Solar Sail with Constant Sail Setting", American Rocket Society Journal, Vol. 30, 1960, pp. 198200 .

Lui, Willian, Rankin, Robert, and Boteler, David, "Space Weather in Canada: A Plan of Action Under LTSP III", Canadian Space Agency Report, April 19, 1999.

Macdonald, Malcolm, GeoSail: Exploring Geospace Using a Small Solar Sail", Solar Sail Mission Applications Seminar, London, England, 10 May 2002.

MacNeal, Richard H., "The Heliogyro, An Interplanetary Flying Machine", NASA CR84460, Astro Research Corporation, June 1967.

MacNeal, Richard H., "Structural Dynamics of the Heliogyro", NASA CR-1745, MacNeal-Schwendler Corporation, May 1971.

MacNeal, Richard H. and Hedgepeth, John M., "Helicopters for Interplanetary Space Flight", $34^{\text {th }}$ Annual National Forum of the American Helicopter Society, Washington, D.C., May 1978.

Mashkevich, S.V. \& Shvartsburg, A.A., "Best" Solar Sail for Heliocentric Halos", Soviet Physics Doklady, Vol. 37, No. 6, June 1992, pp. 290-293.

Matloff, G. L., "Applying International Space Station (ISS) and Solar-Sail Technology to the Exploration and Diversion of Small, Dark near Earth Objects (NEO's)", Acta Astronautica, Vol. 44, No. 2-4, 1999, pp. 151-157.

Matloff, G. L., "Interstellar Solar Sailing: Consideration of Real and Projected Sail Materials", Journal of the British Interplanetary Society, Vol. 37, 1984, pp. 135-141.

Matloff, G. L., \& Mallove, E., "The Interstellar Solar Sail - Optimization and Further Analyses", Journal of the British Interplanetary Society, Vol. 36, 1983, pp. 201-209. 
McFarland, C. A., \& Leipold, M., "Main Belt Asteroid Rendezvous Missions Using Solar Electric and Solar Sail Propulsion", AAS/ALAA Space Flight Mechanics Meeting, Austin, Texas, February 12-15, 1996, pp. 1169-1182.

McInnes, Colin R., Solar Sailing: Technology, Dynamics and Mission Applications, Praxis Publishing Ltd., Chichester, UK, 1999.

McInnes, Colin R., Macdonald, M., Angelopolous, V., Alexander, D., "GEOSAIL: Exploring the Geomagnetic Tail Using a Small Solar Sail", Journal of Spacecraft and Rockets, Vol. 38, No. 4, July - August 2001, pp. 622 629.

McInnes, Colin R., "Near-Term, Low Cost Missions for Solar Sails", Journal of the British Interplanetary Society, Vol. 53, No. 1-2, 2000, pp. 48-61.

McInnes, Colin R., "Payload Mass Fractions for Minimum-Time Trajectories of Flat and Compound Solar Sails", Journal of Guidance, Control and Dynamics, Vol. 23, No. 6, 2000, pp. 1076-1078.

McInnes, Colin R., "Artificial Lagrange Points for a Partially Reflecting Flat Solar Sail", Journal of Guidance, Control and Dynamics, Vol. 22, No. 1, 1999, pp. 185-187.

McInnes, Colin R., "Passive Control of Displaced Solar Sail Orbits", Journal of Guidance, Control and Dynamics, Vol. 21, No. 6, November-December 1998, pp.975982.

McInnes, Colin R., "Solar Sail Mission Applications for Non-Keplerian Orbits," $3^{\text {rd }} I A A$ International Conference on Low-Cost Planetary Missions, Pasadena, California, 27 April - 1 May 1998, Acta Astronautica Vol. 45, Nos. 4-9, 1999, pp. 567-575.

McInnes, Colin R., "Dynamics, Stability, and Control of Displaced Non-Keplerian Orbits", Journal of Guidance, Control and Dynamics, Vol. 21, No. 5, September-October 1998, pp. 799-805.

McInnes, C. R., McDonald, A. J. C., Simmons, J. F. L., MacDonald, E. W., "Solar Sail Parking in Restricted Three-Body Systems," Journal of Guidance, Control and Dynamics, Vol. 17, No. 2, 1994, pp. 399-406.

McInnes, Colin R., "Solar Sail Trajectories at the Lunar $L_{2}$ Lagrange Point," Journal of Spacecraft and Rockets, Vol. 30, No. 6, 1993, pp.782-784.

McInnes, Colin R., and Simmons, John F. L., "Solar Sail Halo Orbits I: Heliocentric Case," Journal of Spacecraft and Rockets, Vol. 29, No. 4, 1992, pp.466-471.

McInnes, Colin R., and Simmons, John F. L., "Solar Sail Halo Orbits II: Geocentric Case," Journal of Spacecraft and Rockets, Vol. 29, No. 4, 1992, pp.472-479.

McInnes, Colin R., "Solar Sailing: A New Tool For Solar System Research," Vistas in Astronomy, Vol. 34, 1991, pp.369-408. 
McInnes, Colin R., and Macpherson, Keith, P., "Solar Sail Halo Trajectories: Dynamics and Applications," $42^{\text {nd }}$ Congress of the International Astronautical Federation, Montreal, October 1991.

McInnes, Colin R. and Brown, John C., "The Dynamics of Solar Sails with a Non-point Source of Radiation Pressure," Celestial Mechanics and Dynamical Astronomy, Vol. 49, 1990, pp.249-264.

McInnes, Colin R., and Brown, J. C., "Solar Sail Dynamics with an Extended Source of Radiation Pressure," Acta Astronautica, Vol. 22, 1990, pp.155-160.

Mclnnes, Colin R., and Simmons, John F. L., "Halo Orbits for Solar Sails - Dynamics and Applications," European Space Agency Journal, Vol. 13, No. 3, 1989, pp.229-234.

McInnes, Colin R., "Advanced Trajectories and Mission Applications of Solar Sail Spacecraft," Departmental Report 9420, University of Glasgow, Department of Aerospace Engineering.

McInnes, Colin R., "Artificial Lagrange Points for a Non-Perfect Solar Sail," Departmental Report 9815 , University of Glasgow, Department of Aerospace Engineering.

McInnes, Colin R., "Solar Sail Spacecraft - Historical Development and Mission Applications," Interdisciplinary Science Reviews, Vol. 20, No. 4, 1995, pp.289-301.

Molostov, A.A. \& Shvartsburg, A.A., "Heliocentric Halos for a Solar Sail with Absorption", Soviet Physics Doklady, Vol. 37, No. 3, March 1992, pp. 149-152.

Molostov, A.A. \& Shvartsburg, A.A., "Heliocentric Synchronous Halos for a Solar Sail with Absorption", Soviet Physics Doklady, Vol. 37, No. 4, April 1992, pp. 195-197.

Morrow, E., Scheeres, D.J. and Lubin, D., "Solar Sail Orbit Operations at Asteroids", Journal of Spacecraft and Rockets, Vol. 38, No. 2, March-April 2001, pp. 279-286.

Murray, C.D. and Dermott, S.F., Solar System Dynamics, Cambridge University Press, USA, 1999.

Otten, Michiel and McInnes, Colin R., "Near Minimum-Time Trajectories for Solar Sails", Journal of Guidance Control and Dynamics, Vol. 24, No. 3, 2001, pp. 632-634.

Prado, J.Y., Perret, A., Pignolet, G. \& Dandouras, I., "Using a Solar Sail for a Plasma Storm Early Warning System", IAA-96-IAA.3.3.06, $47^{\text {th }}$ International Astronautical Congress, October 1996, pp1-12.

Rowe, W.M., Luedke, E.E. and Edwards, D.K., "Thermal Radiative Properties of Solar Sail Film Materials", $2^{\text {nd }}$ AIAA/ASME Thermophysics and Heat Transfer Conference, Palo Alto, California, May 24-26, 1978, pp. 1-8. 
Sackett, Lester L. \& Edelbaum, Theodore N., "Optimal Solar Sail Spiral to Escape", AAS/AIAA Conference, Grand Teton National Park, Wyoming, September 2-9 1977, pp. $1-32$.

Sands, Norman, "Escape From Planetary Gravitational Fields by Use of Solar Sails", American Rocket Society Journal, Vol. 31, April 1961, pp. 527-531.

Sauer, Carl G., "Optimum Solar Sail Interplanetary Trajectories", AIAA/AAS Astrodynamics Conference, San Diego, CA, August 18-20, 1976, pp. 1-8.

Scaglione, S., \& Vulpetti, G., "The Aurora Project: Removal of Plastic Substrate to Obtain an All-Metal Solar Sail", Acta Astronautica, Vol. 44, No. 2-4, 1999, pp. 147-150.

Shyartsburg, A.A., "Three-Dimensional Solar Sails: Passive Stability of Levitation and Slow Orbital Motions", Cosmic Research, Vol. 35, No. 2, 1997, pp. 183-187.

Shvartsburg, A.A., "Geocentric Halos for a Solar Sail with Absorption", Soviet Physics Doklady, Vol. 38, No. 2, February 1993, pp. 85-88.

Simon, K. and Zakharov, Y., "Optimization of Interplanetary Trajectories with Solar Sail”, Space Technology, Vol. 16, No. 5/6, 1996, pp. 381-385.

Sonntag, Richard E. and Van Wylen, Gordon, Introduction to Thermodynamics Classical and Statistical $2^{\text {nd }}$ Edition, John Wiley \& Sons Inc., USA, 1971.

Subba Rao, P.V. \& Ramanan, R.V., "Optimal Three-Dimensional Heliocentric Solar-Sail Rendezvous Transfer Trajectories", Acta Astronautica Vol. 29, No. 5, 1993, pp. 341-345.

Tascione, Thomas F., Introduction to the Space Environment, Orbit Book Company, Inc., U.S.A., 1988.

Tsu, T.C., "Interplanetary Travel by Solar Sail", American Rocket Society Journal, Vol. 29,1959, pp. 422-427.

Van der Ha, J.C. \& Modi, V.J., "Long-Term Evaluation of Three-Dimensional Heliocentric Solar Sail Trajectories with Arbitrary Fixed Sail Setting", Celestial Mechanics, Vol. 19, 1979, pp. 113-138.

Vulpetti, Giovanni, "3D High-Speed Escape Heliocentric Trajectories by All-MetallicSail Low-Mass Sailcraft”, Acta Astronautica, Vol. 39, No. 1-4, 1996, pp. 161-170.

Vulpetti, Giovanni, \& Scaglioine, S., "The Aurora Project: Estimation of the Optical Sail Parameters", Acta Astronautica, Vol. 44, No. 2-4, 1999, pp. 123-132.

Williams, Trevor \& Collins, Patrick, "Attitude Control System and Communications Payload for an Amateur Solar Sail Spacecraft" IAF 84-363, 1984. 
Wright, Jerome L., Space Sailing, Gordon and Breach Science Publishers, The Netherlands, 1992. 